\title{
Replenishment and Fulfillment Based Aggregation for General Assemble-to-Order Systems
}

\author{
Emre Nadar, Alp Akçay \\ Department of Industrial Engineering, Bilkent University, 06800 Ankara, Turkey, \{emre.nadar, alp.akcay\}@bilkent.edu.tr \\ Mustafa Akan, Alan Scheller-Wolf \\ Tepper School of Business, Carnegie Mellon University, Pittsburgh, Pennsylvania 15213, \{akan, awolf\}@andrew.cmu.edu
}

We consider an assemble-to-order system with multiple products, multiple components which may be demanded in different quantities by different products, batch ordering of components, random lead times, and lost sales. We model the system as an infinite-horizon Markov decision process under the discounted cost criterion. A control policy specifies when a batch of components should be produced (i.e., inventory replenishment) and whether an arriving demand for each product should be satisfied (i.e., inventory allocation). As optimal solutions for such problems are computationally intractable for even moderate sized systems, we approximate the optimal cost function by reducing the state space of the original problem via a novel aggregation technique that uses knowledge of products' component requirements and components' replenishment batch sizes.

We establish that a lattice-dependent base-stock and lattice-dependent rationing policy is the optimal inventory replenishment and allocation policy for the aggregate problem under a disaggregation rule that disaggregates each aggregate state into its two extreme original states. This rule drastically reduces the per iteration computational complexity of the value iteration algorithm for the aggregate problem (without sacrificing much accuracy, according to our numerical experiments). We further alleviate the value iteration computational burden by eliminating suboptimal actions based on our optimal policy structure.

For systems in which there is a product that has fulfillment priority over all other products at optimality, we are able to derive finite error bound for the cost function of the aggregate problem. With these bounds we show that the value iteration algorithm in the original problem that starts with the aggregate solution converges to the optimal cost function. Numerical experiments indicate that such an algorithm has distinct computational advantage over the standard value iteration method in the original problem.

Key words: assemble-to-order systems; Markov decision processes; approximate dynamic programming; aggregation

\section{Introduction}

Assemble-to-order (ATO) systems appear in many industries where rapid delivery of customized products or multi-item orders plays a vital role; they are particularly popular in automotive, consumer electronics, and online retailing industries. (Kapuscinski et al., 2004, Xu et al., 2009, and Lu et al., 2014, provide specific examples from these industries.) ATO production holds inventory at the component level so that a product may be assembled from its components - if sufficient inventory exists - immediately after a customer demand for this product occurs. This strategy allows the producer to offer greater product variety by providing flexibility in the use of potentially 
scarce components. ATO systems are thus more appealing when components are expensive or when replenishment lead times are much longer than the assembly time (Bernstein et al. 2011).

ATO systems have received much attention in the literature; Song and Zipkin (2003) provide a comprehensive review. More recently, several authors have identified the optimal and/or asymptotically optimal policy structure for managing inventory in very specific ATO systems: Lu et al. (2010) establish that no-holdback (NHB) allocation rules are optimal for generalized $W$-systems operating under an independent base-stock (IBS) replenishment policy when the "symmetric cost" condition holds. Doğru et al. (2010) prove that an IBS policy and an NHB allocation policy with a priority based backorder clearing (PBC) rule are optimal for $W$-systems with identical component lead times when the "symmetric cost" or "balanced capacity" condition holds. Reiman and Wang (2012) extend the results of Doğru et al. (2010) to generalized $W$-systems with symmetric costs when all unique components have the same lead time, which is longer than that of the common component. Lu et al. (2014) show that a coordinated base-stock (CBS) policy and NHB allocation rules are optimal for $N$ - and $W$-systems with non-identical replenishment lead times and symmetric costs. They also reveal the asymptotic optimality of a CBS policy and an NHB policy with a PBC rule under high demand volume and asymmetric costs.

For ATO systems with identical component lead times, Reiman and Wang (2015) prove the asymptotic optimality of an IBS policy combined with a stochastic program based allocation rule as the lead time goes to infinity. Doğru et al. (2014) leverage the allocation rule of Reiman and Wang (2015) to generate results for $M$-systems: a stock reservation policy is asymptotically optimal if the inventory cost of the assembled product exceeds the sum of those of individual products, and an NHB policy with a myopic priority rule is asymptotically optimal otherwise. Wan and Wang (2015) prove the asymptotic optimality of the allocation rule in Reiman and Wang (2015) under high demand volume, showing that stock reservation is necessary to achieve asymptotic optimality in many systems.

For ATO systems with lost sales, researchers have obtained optimality results under Markovian assumptions on production and demand: Benjaafar and ElHafsi (2006) establish the optimality of a state-dependent base-stock and state-dependent rationing (SBSR) policy for an ATO assembly system with multiple demand classes. ElHafsi (2009) extends the results of Benjaafar and ElHafsi (2006) by allowing customer orders to arrive as a compound Poisson process. ElHafsi et al. (2008) prove that an SBSR policy is optimal for an ATO system with a nested product structure. Benjaafar 
et al. (2011) show that an SBSR policy is optimal for an ATO assembly system with multiple stages, each producing a different item in batches of variable sizes. And Nadar et al. (2014) establish that a lattice-dependent base-stock and lattice-dependent rationing (LBLR) policy is optimal for generalized $M$-systems. Nadar et al. (2015) numerically demonstrate the optimal performance of LBLR for ATO systems with general product structures.

Despite the great interest from both academia and industry, however, the optimal policy structure is still unknown in general ATO systems. Identifying such optimal policies is a significant challenge, as such a policy needs to address both replenishment and allocation issues for an arbitrary number of components and products assembled with general product structures. Consequently, several papers focus on various heuristic policies that may be usefully employed in practice: Akçay and $\mathrm{Xu}(2004)$ demonstrate the practicality of an order based component allocation rule in a periodicreview ATO system with response time windows and the IBS policy. They optimize the base-stock levels based on sample average approximation (SAA). Huang (2014) evaluates the use of lastcome-first-served (within one period) and product based priority (within time windows) rules for component allocation in a periodic-review ATO system with the IBS policy. Several other papers study the optimization of IBS or independent $(s, S)$ policies in continuous-review ATO systems with the first-come-first-served (FCFS) allocation rule. See, for example, Lu and Song (2005), Lu et al. (2005), Zhao and Simchi-Levi (2006), and van Jaarsveld and Dollevoet (2011). Finally, van Jaarsveld and Scheller-Wolf (2015) develop an SAA algorithm that computes near-optimal base-stock levels in large scale ATO systems with the FCFS allocation rule. They also study the performance of IBS and FCFS policies, comparing their costs to lower bounds on the least costs that can be attained under optimal base-stock replenishment and FCFS allocation policies, and optimal base-stock replenishment and optimal allocation policies.

Thus knowledge of ATO systems is largely restricted to (i) optimal policies for specific systems like the $M$ or $W$, and (ii) performance evaluation and optimization techniques for heuristic policies for general problems. This study presents a new approach to optimizing general ATO systems by approximating the problem via aggregation of the state space, without restricting the action space to any heuristic policy.

Specifically, we consider continuous-time ATO systems with general product structures. We model the problem as an infinite-horizon Markov decision process (MDP) with state space consisting of component inventory levels. Each component is produced in batches of fixed size in a maketo-stock fashion, production times are independent and exponentially distributed, and demand for 
each product arrives as an independent Poisson process. If not satisfied immediately upon arrival, these demands are lost. A control policy specifies when to produce a batch of any component and whether or not to fulfill an arriving demand if sufficient inventory exists. Solving this ATO problem to optimality is extremely problematic in systems with many components since the state space is unmanageably large; see Nadar et al. (2015) for experimental results in the average cost case. In this study we develop an effective, and computationally efficient, aggregation method to reduce the large state space of the problem.

Aggregation is an approximate dynamic programming method used to provide a good approximation to a value function using a smaller state space, which may be usefully employed in solving large-scale MDP problems. We refer the reader to Tsitsiklis and Van Roy (1996), Chapter 6 in Heyman and Sobel (2003), Chapter 8 in Powell (2011), and Chapter 6 in Bertsekas (2012) for in-depth discussions of value function approximations via aggregation. See also Rogers et al. (1991) for a comprehensive survey of aggregation methods in optimization.

A few researchers have studied the aggregation technique in the ATO literature: Vliegen and van Houtum (2009) use this technique in the so-called service tool problem with joint returns and partial order service. In this problem several service tools are needed to perform a maintenance action. After usage all tools are returned together, leading to a Markov chain with a very large state space. They propose an approximation in which all states with the same number of tools in the return pipeline are aggregated, ignoring the sets the tools were demanded in. Busic et al. (2012) use aggregation with an appropriate modification of the transition probabilities in the original Markov chain, in order to construct bounding chains with a common state space of reduced cardinality. They also apply their method in the service tool problem, deriving provable bounds on the average value of the order fill rate under the assumption of a base stock policy for each component. More recently, Busic and Coupechoux (2014) take a similar approach to derive bounding chains for an original Markov chain, and then use them as inputs in a perfect simulation algorithm for the purpose of drawing samples from the exact stationary distribution of the original Markov chain. All of the above papers focus on the performance evaluation of a given policy, while we use state aggregation in search of an optimal policy in a general ATO system. We refer the reader to Kemeny and Snell (1976) for further details on state aggregation in Markov chains.

Our aggregation technique partitions the original state space into disjoint subsets based on knowledge of products' component requirements and components' replenishment batch sizes. Each 
subset consists of the same number of original states, forming an "aggregate" state in the aggregate problem. Note that an aggregate state may contain an arbitrary number of original states. Our aggregation scheme specifies that the system stays in the same aggregate state or moves to an adjacent aggregate state upon replenishment of a batch of any component, or upon fulfillment of a demand for any product, cf. Definition 1 in Section 3. After defining our aggregation, we formulate the optimality equation for the aggregate problem: In a given aggregate state, the controller disaggregates the aggregate state into its original states according to a specific probability distribution. The system is then again subject to the admissible action space of the original problem, and moves between original states as in the original problem. However, the controller determines the optimal actions based on the cost function evaluated not in those original states, but in the corresponding aggregate states.

The probability distribution used to disaggregate an aggregate state into its original states (i.e., the disaggregation probabilities) has the potential to greatly influence the performance of our aggregation scheme. In this paper we develop a fulfillment based disaggregation scheme that assigns zero probability to certain original states in a given aggregate state, cf. Definition 2 in Section 3: In our disaggregation scheme the disaggregation probability of a particular original state can be positive only if the system moves to a lower aggregate state (in each dimension) upon fulfillment of a demand for any product, or if the aggregate state remains the same upon fulfillment of a demand for any product.

Disaggregating each aggregate state into its two extreme original states (the smallest and the largest states) is one possible rule that obeys our disaggregation scheme, when aggregate states are sufficiently large. This rule proves very effective in our numerical experiments: If we take the average percentage deviation from optimal cost across all states as our performance criterion, the average distance of our aggregate solution from the optimal solution is $8.49 \%$ under the above rule, on a test bed of 150 instances. Inspired by Rogers and Plante (1993), we also consider an alternative (uniform) rule that assigns equal probabilities to all original states of an aggregate state and thus violates our disaggregation scheme. The average distance of our aggregate solution from the optimal solution becomes $8.92 \%$ under the latter rule, on the same test bed. But, if we take the percentage deviations from optimal cost across all states weighted by optimal stationary distribution, the average distances are $15.03 \%$ and $20.56 \%$, respectively. And if we take the percentage deviation from the optimal cost evaluated in the optimal state, the average distances are $17.05 \%$ and $26.85 \%$, respectively. 
Our rule also drastically reduces the per iteration computational complexity of the standard value iteration algorithm that we employ to solve the aggregate problem: On our test bed, the computation time of the aggregate problem under our rule is on average $95.8 \%$ lower than that of the original problem when solved by value iteration. However, the aggregate problem under the uniform rule is on average computationally less attractive than the original problem.

Furthermore, our disaggregation scheme (in Definition 2) allows us to establish the optimality of an LBLR policy for the aggregate problem, cf. Theorem 1: The original state space of the problem can be partitioned into disjoint lattices such that, on each lattice, (a) it is optimal to produce a batch of a particular component if and only if the current original state (within the aggregate state) is less than the base-stock level of that component on the current lattice; and (b) it is optimal to fulfill a demand for a particular product if and only if the current original state (within the aggregate state) is greater than or equal to the rationing level for that product on the current lattice.

We use this optimal policy structure to further reduce the computational burden of the value iteration algorithm in the aggregate problem: On a given iteration, once the optimal actions in any state are found, we eliminate from consideration all actions in each remaining state that are not consistent with an LBLR policy when combined with the optimal actions revealed thus far. This action elimination procedure reduces the already-low computation time of the aggregate problem under our disaggregation scheme by a further $43.4 \%$, on average, on our test bed of 150 instances.

Finally, we consider the case when there is a product that should have fulfillment priority over all other products in the original problem. For this case we derive a finite error bound for the cost function of our aggregate problem, cf. Theorem 2. We use this bound to construct a value iteration algorithm that starts with the cost function of our aggregate problem and prove that it converges to the optimal cost function of the original problem, cf. Theorem 3. If such an algorithm is implemented on our test bed, the maximum distance from the optimal cost across all states drops to $5 \%$ and $1 \%$ with, on average, $54 \%$ and $38 \%$ fewer iterations, respectively, in comparison with the standard value iteration algorithm.

We thus contribute to the ATO literature in several important ways:

- To our knowledge, we are the first to study state aggregation in the optimization of ATO systems, highlighting its practicality when certain product and component characteristics are incorporated into aggregation/disaggregation schemes. 
- We identify the optimal policy structure in our aggregate problem for general ATO systems. Much of the complexity in the ATO problem is attributable to the simultaneous consideration of inventory replenishment and allocation decisions. The problem becomes even more difficult when replenishment and/or allocation decisions involve non-unitary changes in several component inventory levels, as in our study. Our aggregation technique enables a clean analytical formulation to handle such complexity: it renders the state space separable into multiple disjoint lattices so that well-known threshold policies (base-stock and rationing) are optimal on each of those lattices. This result allows us to restrict the search for an optimal control policy to the class of LBLR policies in the aggregate problem, dramatically reducing the computational requirements of the value iteration algorithm.

- We find a finite error bound for the aggregate problem under a mild "dominant product" condition. This allows us to validate the use of a value iteration method that starts with the aggregate solution in the original problem. Numerical results indicate this method is computationally more efficient than the standard value iteration method.

The rest of the paper is organized as follows: Section 2 describes the original problem. Section 3 describes the aggregate problem along with our disaggregation scheme. Section 4 establishes the optimal policy structure for the aggregate problem under our disaggregation scheme. Section 5 offers an error bound for the aggregate problem under a mild condition and establishes the convergence of the value iteration algorithm that starts with the aggregate solution. Section 6 presents our numerical results. Section 7 offers a summary and concludes. All proofs are contained in an online appendix.

\section{The Original Problem}

We consider an ATO system with $m$ components $(i=1,2, . ., m)$ and $n$ products $(j=1,2, . ., n)$. Define $\mathbf{A}$ as an $m \times n$ nonnegative resource-consumption matrix; $a_{i j}$ is the number of units of component $i$ needed to assemble one unit of product $j$, and $\mathbf{a}_{j}$ is the $j$ th column of $\mathbf{A}$. Each component $i$ is produced in batches of a fixed size $q_{i}$ in a make-to-stock fashion. Define $\mathbf{q}=$ $\left(q_{1}, q_{2}, . ., q_{m}\right)$ as the vector of production batch sizes. Production time for a batch of component $i$ is independent of the system state and the number of outstanding orders of any type, and exponentially distributed with finite mean $1 / \mu_{i}$. Assembly lead times are negligible so that assembly operations can be postponed until demand is realized. Demand for each product $j$ arrives as an independent Poisson process with finite rate $\lambda_{j}$. Demand for product $j$ can be fulfilled only if all 
the required components are available; otherwise, the demand is lost, incurring a unit lost sale cost $c_{j}$. Demand may also be rejected in the presence of all the necessary components, again incurring the unit lost sale cost $c_{j}$. Many papers model the ATO problem under the above or more restrictive assumptions (see, for example, Ha 1997, Benjaafar and ElHafsi 2006, ElHafsi et al. 2008, and Nadar et al. 2014).

The state of the system at time $t$ is the vector $\mathbf{X}(t)=\left(X_{1}(t), . ., X_{m}(t)\right)$, where $X_{i}(t)$ is a nonnegative integer denoting the on-hand inventory for component $i$ at time $t$. Component $i$ held in stock incurs a unit holding cost per unit time $h_{i}>0$. Denote by $h(\mathbf{X}(t))=\sum_{i} h_{i} X_{i}(t)$ the total inventory holding cost rate in state $\mathbf{X}(t)$. Define $t_{k}$ as the time of occurrence of the $k$ th state transition. Also let $t_{0}=0$. The state of the system stays constant between transitions, i.e., $\mathbf{X}(t)=\mathbf{X}\left(t_{k}\right)=\left(X_{1}\left(t_{k}\right), . ., X_{m}\left(t_{k}\right)\right)$ for $t_{k} \leq t<t_{k+1}$. Since all inter-event times are exponentially distributed, the system retains no memory, and thus optimality equations remain the same for all $t$ such that $t_{k} \leq t<t_{k+1}$. This implies that the optimal decision stays the same between transitions, and decision epochs can be restricted to times when the state changes.

We thus formulate the problem as an MDP and focus on Markovian policies for which actions at each decision epoch depend solely on the current state. A control policy $\ell$ specifies for each state $\mathbf{x}=\left(x_{1}, . ., x_{m}\right)$, the action $\mathbf{u}^{\ell}(\mathbf{x})=\left(u^{(1)}, . ., u^{(m)}, u_{1}, . ., u_{n}\right), u^{(i)}, u_{j} \in\{0,1\}, \forall i, j ;$ where $u^{(i)}=1$ means produce component $i$, and $u^{(i)}=0$ means do not produce component $i ; u_{j}=1$ means satisfy demand for product $j$, and $u_{j}=0$ means reject demand for product $j$. Denote by $\mathbb{U}(\mathbf{x})$ the set of admissible actions in state $\mathbf{x}$. For any action $\mathbf{u}=\left(u^{(1)}, . ., u^{(m)}, u_{1}, . ., u_{n}\right) \in \mathbb{U}(\mathbf{x})$, we must have $u_{j}=0$ if $\exists i$ such that $x_{i}<a_{i j}$.

As each ordering decision specifies only whether or not to produce a component, there is at most one outstanding batch order for each component at any time. Also, as component orders are not part of our system state, these can in effect be cancelled upon transition to a new state. Both of these assumptions are standard in the literature (again see, for example, Ha 1997, Benjaafar and ElHafsi 2006, ElHafsi et al. 2008, and Nadar et al. 2014).

Let $v$ denote a real-valued function defined on $\mathbb{N}_{0}^{m}\left(\mathbb{N}_{0}\right.$ is the set of nonnegative integers and $\mathbb{N}_{0}^{m}$ is its $m$-dimensional cross product). Also define $0<\alpha<1$ as the discount parameter. For a given policy $\ell=\tilde{\ell}$ and a starting state $\mathbf{X}(0)=\mathbf{x}$, the expected discounted cost over an infinite planning horizon, $v^{\widetilde{\ell}}(\mathbf{x})$, can be written as

$$
v^{\widetilde{\ell}}(\mathbf{x})=E\left[\int_{0}^{\infty} e^{-\alpha t} h(\mathbf{X}(t)) d t+\sum_{j=1}^{n} \int_{0}^{\infty} e^{-\alpha t} c_{j} d N_{j}(t) \mid \mathbf{X}(0)=\mathbf{x}, \ell=\widetilde{\ell}\right]
$$


where $N_{j}(t)$ is the cumulative number of demands for product $j$ that have not been fulfilled from on-hand inventory up to time $t$.

The time between the transition to state $\mathbf{x}$ and the transition to the next state is exponentially distributed with rate $\nu_{\mathbf{x}}(\mathbf{u})$ if action $\mathbf{u}=\left(u^{(1)}, . ., u^{(m)}, u_{1}, . ., u_{n}\right) \in \mathbb{U}(\mathbf{x})$ is selected in state $\mathbf{x}$. Following Lippman (1975), we consider a uniformized version of the problem where the rate of transition $\nu$ is an upper bound for all states and controls, i.e., $\nu \geq \nu_{\mathbf{x}}(\mathbf{u}), \forall \mathbf{x}, \mathbf{u}$. Specifically, we will formulate the problem for the choice $\nu=\sum_{i} \mu_{i}+\sum_{j} \lambda_{j}$. Thus the $k$ th transition time interval $\left(t_{k+1}-t_{k}\right)$ is exponentially distributed with rate $\nu, \forall k$. With the uniform transition rate, we are able to transform the continuous-time control problem into an equivalent discrete-time control problem.

If action $\mathbf{u}=\left(u^{(1)}, . ., u^{(m)}, u_{1}, . ., u_{n}\right) \in \mathbb{U}(\mathbf{x})$ is selected in state $\mathbf{x}$, the next state is $\tilde{\mathbf{x}}$ with probability $p_{\mathbf{x}, \tilde{\mathbf{x}}}(\mathbf{u})$. Thus:

$$
p_{\mathbf{x}, \tilde{\mathbf{x}}}(\mathbf{u})= \begin{cases}\frac{\mu_{i} u^{(i)}}{\nu} & \text { if } \tilde{\mathbf{x}}=\mathbf{x}+q_{i} e_{i}, \\ \frac{\lambda_{j} u_{j}}{\nu} & \text { if } \tilde{\mathbf{x}}=\mathbf{x}-\mathbf{a}_{j}, \\ \frac{\nu-\sum_{i=1}^{m} \mu_{i} u^{(i)}-\sum_{j=1}^{n} \lambda_{j} u_{j}}{\nu} & \text { if } \tilde{\mathbf{x}}=\mathbf{x}, \text { and } \\ 0 & \text { otherwise, }\end{cases}
$$

where $e_{i}$ is the $i$ th unit vector of dimension $m$ ( $\mathbf{e}$ is an $m$-dimensional vector of ones). In this discrete-time framework, $N_{j}\left(t_{k}\right)$ is the cumulative number of unsatisfied demands for product $j$ at the time of the $k$ th transition, and $h\left(\mathbf{X}\left(t_{k}\right)\right)$ is the total inventory holding cost rate during the time interval $\left[t_{k}, t_{k+1}\right)$. Then, $v^{\widetilde{\ell}}(\mathbf{x})$ in (1) can be rewritten as follows. (See Benjaafar and ElHafsi, 2006, and Nadar et al., 2014, for similar formulations in the ATO literature.)

$$
v^{\widetilde{\ell}}(\mathbf{x})=E\left[\sum_{k=0}^{\infty}\left(\frac{\nu}{\alpha+\nu}\right)^{k} \frac{h\left(\mathbf{X}\left(t_{k}\right)\right)}{\alpha+\nu}+\sum_{k=1}^{\infty}\left(\frac{\nu}{\alpha+\nu}\right)^{k} \cdot \sum_{j=1}^{n} c_{j}\left(N_{j}\left(t_{k}\right)-N_{j}\left(t_{k-1}\right)\right) \mid \mathbf{X}(0)=\mathbf{x}, \ell=\tilde{\ell}\right] .
$$

Our objective is to identify a policy $\ell^{*}$ that minimizes the expected discounted cost. We below formulate the optimality equation that holds for the optimal cost function $v^{*}=v^{\ell^{*}}$ :

$$
v^{*}(\mathbf{x})=\min _{\mathbf{u} \in \mathbb{U}(\mathbf{x})}\left\{\frac{h(\mathbf{x})}{\alpha+\nu}+\left(\frac{\nu}{\alpha+\nu}\right) \sum_{j=1}^{n} \frac{\lambda_{j} c_{j}\left(1-u_{j}\right)}{\nu}+\left(\frac{\nu}{\alpha+\nu}\right) \sum_{\tilde{\mathbf{x}}} p_{\mathbf{x}, \tilde{\mathbf{x}}}(\mathbf{u}) v^{*}(\tilde{\mathbf{x}})\right\} .
$$

Therefore, our continuous-time control problem is equivalent to a discrete-time control problem with discount factor $\nu /(\alpha+\nu)$ and cost per stage given by

$$
\frac{h(\mathbf{x})}{\alpha+\nu}+\left(\frac{\nu}{\alpha+\nu}\right) \sum_{j=1}^{n} \frac{\lambda_{j} c_{j}\left(1-u_{j}\right)}{\nu} .
$$


As it is always possible to redefine the time scale, without loss of generality we assume $\alpha+\nu=1$. Then the optimality equation in (3) can be simplified as follows:

$$
v^{*}(\mathbf{x})=h(\mathbf{x})+\sum_{i} \mu_{i} T^{(i)} v^{*}(\mathbf{x})+\sum_{j} \lambda_{j} T_{j} v^{*}(\mathbf{x}),
$$

where the operator $T^{(i)}$ for component $i$ is defined as

$$
T^{(i)} v(\mathbf{x})=\min \left\{v\left(\mathbf{x}+q_{i} e_{i}\right), v(\mathbf{x})\right\}
$$

and the operator $T_{j}$ for product $j$ is defined as

$$
T_{j} v(\mathbf{x})= \begin{cases}\min \left\{v(\mathbf{x})+c_{j}, v\left(\mathbf{x}-\mathbf{a}_{j}\right)\right\} & \text { if } \mathbf{x} \geq \mathbf{a}_{j} \\ v(\mathbf{x})+c_{j} & \text { otherwise }\end{cases}
$$

For a given state $\mathbf{x}$, the operator $T^{(i)}$ specifies whether or not to produce a batch of component $i$, and the operator $T_{j}$ specifies, upon arrival of a demand for product $j$, whether or not to fulfill it from inventory, if sufficient inventory exists.

As a computational requirement, we will restrict the state space to be finite; define $\overline{\mathbf{x}}=\left(\bar{x}_{1}, . ., \bar{x}_{m}\right)$ as a vector of upper bounds for component inventory levels, and $\mathcal{X}$ as the set of system states. Thus, for any state $\mathbf{x} \in \mathcal{X}$ at any time, we must have $0 \leq x_{i} \leq \bar{x}_{i}, \forall i$. Also, for any action $\mathbf{u}=$ $\left(u^{(1)}, . ., u^{(m)}, u_{1}, . ., u_{n}\right) \in \mathbb{U}(\mathbf{x})$, we must have $u^{(i)}=0$ if $x_{i}+q_{i}>\bar{x}_{i}$. Note that the upper bounds should be sufficiently high so that they are never visited at optimality.

\section{The Aggregate Problem}

In this section we will use "hard aggregation" to approximate the value function of our ATO problem in Section 2. In hard aggregation we group the original system states into disjoint nonempty subsets; each such subset forms an "aggregate" state, and each original state belongs to only one aggregate state. Define $\mathcal{Y}$ as the set of aggregate states. Denote $\mathbf{x} \in \mathbf{y}$ if the original state $\mathbf{x}$ belongs to aggregate state $\mathbf{y}$, and for every $\mathbf{x}$ denote by $\mathbf{y}(\mathbf{x})$ the aggregate state $\mathbf{y}$ with $\mathbf{x} \in \mathbf{y}$. For each aggregate state $\mathbf{y}$ and each original state $\mathbf{x}$, we introduce the disaggregation probability $d_{\mathbf{y x}}$ as the degree to which $\mathbf{y}$ is represented by $\mathbf{x}$. In a hard aggregation scheme:

$$
\sum_{\mathbf{x} \in \mathbf{y}} d_{\mathbf{y x}}=1, \quad \forall \mathbf{y} \in \mathcal{Y}
$$

In other words, in hard aggregation, the disaggregation probabilities $d_{\mathbf{y x}}$ are restricted to be zero for states $\mathbf{x}$ that are not in aggregate state $\mathbf{y}$.

Define $\mathbf{b}=\left(b_{1}, . ., b_{m}\right)$ as a vector of positive integers such that $b_{i} \geq \max \left\{q_{i}, \max _{j}\left\{a_{i j}\right\}\right\}, \forall i$. With this we now formally define the aggregation scheme that we consider in this and subsequent sections. 
Definition 1. (Replenishment and fulfillment based aggregation.) The aggregate state $\mathbf{y}(\mathbf{x})=\left(y_{1}, . ., y_{m}\right)$ is constructed as follows.

$$
y_{i}=\left\lfloor\frac{x_{i}}{b_{i}}\right\rfloor, \forall i
$$

i.e., $x_{i}=b_{i} y_{i}+z_{i}$ where $z_{i} \in\left\{0,1, . ., b_{i}-1\right\}, \forall i$.

An illustration of our aggregation scheme for a 2-component 2-product system is shown in Figure 1. If $\mathbf{b}$ is defined minimally in our aggregation scheme, i.e., $b_{i}=\max \left\{q_{i}, \max _{j}\left\{a_{i j}\right\}\right\}$, two original states map to different aggregate states if either the inventory levels of some component $i$ at these original states are at least one replenishment batch apart, or if the same number of units of some product $j$ cannot be made from on-hand inventory of component $i$ in these original states (assuming an ample supply for all the other components).

Our aggregation scheme implies that the size of the state space decreases as $b_{i}$ increases. Define $\overline{\mathbf{y}}=\left(\bar{y}_{1}, . ., \bar{y}_{m}\right)$ as a vector of upper bounds for aggregate state variables. Thus, for any aggregate state $\mathbf{y} \in \mathcal{Y}$ at any time, we must have $0 \leq y_{i} \leq \bar{y}_{i}, \forall i$. We choose our upper bounds for component inventory levels in the original problem as follows.

$$
\bar{x}_{i}+1=b_{i}\left(\bar{y}_{i}+1\right), \forall i
$$

As a result, each aggregate state contains the same number of original states. In particular, there are $\prod_{i} b_{i}$ original states in each aggregate state, i.e., the size of the state space is reduced by a factor of $\prod_{i} b_{i}$.

We below formulate the Bellman equations that hold for the optimal cost function approximation $r^{*}$ under our aggregation scheme (see Chapter 6 in Bertsekas 2012 for a detailed explanation):

$$
r^{*}(\mathbf{y})=\sum_{\mathbf{x} \in \mathbf{y}} d_{\mathbf{y} \mathbf{x}} \tilde{v}(\mathbf{x}), \quad \mathbf{y} \in \mathcal{Y},
$$

where $\tilde{v}(\mathbf{x})$ is the optimal cost-to-go from original state $\mathbf{x}$ that is generated from aggregate state $\mathbf{y}$ as in Definition 1. The function $\tilde{v}$ is defined as

$$
\tilde{v}(\mathbf{x})=h(\mathbf{x})+\sum_{i} \mu_{i} \tilde{v}^{(i)}(\mathbf{x})+\sum_{j} \lambda_{j} \tilde{v}_{j}(\mathbf{x})
$$

where the function $\tilde{v}^{(i)}$ for component $i$ is given by

$$
\tilde{v}^{(i)}(\mathbf{x})=\min \left\{r^{*}\left(\mathbf{y}\left(\mathbf{x}+q_{i} e_{i}\right)\right), r^{*}(\mathbf{y}(\mathbf{x}))\right\}, \quad \forall i \in\{1, . ., m\}
$$


and the function $\tilde{v}_{j}$ for product $j$ is given by

$$
\tilde{v}_{j}(\mathbf{x})=\left\{\begin{array}{ll}
\min \left\{r^{*}(\mathbf{y}(\mathbf{x}))+c_{j}, r^{*}\left(\mathbf{y}\left(\mathbf{x}-\mathbf{a}_{j}\right)\right)\right\} & \text { if } \mathbf{x} \geq \mathbf{a}_{j}, \\
r^{*}(\mathbf{y}(\mathbf{x}))+c_{j} & \text { otherwise, }
\end{array} \quad \forall j \in\{1, . ., n\} .\right.
$$

Once $r^{*}$ is computed, a suboptimal policy can be found through the minimizations in (9) and (10). Throughout the paper, we will use the term "aggregate-optimal cost function" to denote the function $r^{*}$, and the term "aggregate-optimal policy" to denote the suboptimal policy (for the original problem) obtained from the function $r^{*}$. We now introduce the following rule for disaggregation probabilities in (7).

Definition 2. (Fulfillment based disaggregation.) For $\mathbf{x} \in \mathbf{y}$, the disaggregation probability $d_{\mathbf{y x}}$ equals zero if at least one of the following two conditions hold:

(1) $\exists i$ s.t. $\min _{j}\left\{a_{i j}\right\}+b_{i}\left\lfloor\frac{x_{i}}{b_{i}}\right\rfloor \leq x_{i}<\max _{j}\left\{a_{i j}\right\}+b_{i}\left\lfloor\frac{x_{i}}{b_{i}}\right\rfloor$, or

(2) $\exists i, k$ s.t. $x_{i}<\min _{j}\left\{a_{i j}\right\}+b_{i}\left\lfloor\frac{x_{i}}{b_{i}}\right\rfloor$ and $\max _{j}\left\{a_{k j}\right\}+b_{k}\left\lfloor\frac{x_{k}}{b_{k}}\right\rfloor \leq x_{k}$.

Also, for $\mathbf{x}_{1} \in \mathbf{y}_{1}$ and $\mathbf{x}_{2} \in \mathbf{y}_{2}, d_{\mathbf{y}_{1} \mathbf{x}_{1}}=d_{\mathbf{y}_{2} \mathbf{x}_{2}}$ if $x_{1, i}-b_{i} y_{1, i}=x_{2, i}-b_{i} y_{2, i}, \forall i$, or equivalently, $d_{\mathbf{y x}}=d_{\mathbf{z}}$ where $x_{i}=b_{i} y_{i}+z_{i}, 0 \leq z_{i}<b_{i}, \forall i$, and $\mathbf{z}=\left(z_{1}, . ., z_{m}\right), \forall \mathbf{y}$, and $\forall \mathbf{x} \in \mathbf{y}$.

See Figure 1 for an example. In the most general form of hard aggregation, the disaggregation probabilities $d_{\mathbf{y x}}$ can be positive for any state $\mathbf{x} \in \mathbf{y}$. However, Definition 2 restricts the disaggregation probabilities $d_{\mathbf{y x}}$ to be positive only for a certain subset of states $\mathbf{x} \in \mathbf{y}$ : The disaggregation probability of the state $\mathbf{x} \in \mathbf{y}$ can be positive only if the aggregate state $\mathbf{y}(\mathbf{x})$ remains the same upon fulfillment of a demand for product $j, \forall j$, or the aggregate state $\mathbf{y}(\mathbf{x})$ drops to $\mathbf{y}(\mathbf{x})-\mathbf{e}$ upon fulfillment of a demand for product $j, \forall j$.

Our disaggregation scheme has several desirable characteristics. First, it may significantly reduce the error introduced by our aggregation method in Definition 1: Suppose that the current original state is in the middle portion of one particular aggregate state, e.g., $\mathbf{x}=(1,1)$ or $(2,1)$ in Figure 1. If a batch of component is ordered in (9) or a demand is satisfied in (10), the system will likely stay in the same aggregate state. Because assigning positive disaggregation probabilities to those sub-states in the center of an aggregate state may lead to many such self-transitions, $r^{*}$ may remain the same in many minimizations in (9) and (10), and a very poor approximation results. A simple and effective way to reduce the number of self-transitions is to assign positive disaggregation probabilities to only the lowest and highest original states of each aggregate state; see Figure 2(a) in Section 6.1 for an example. The system moves from the lowest original state of a particular aggregate state to a different aggregate state whenever a demand for any product 


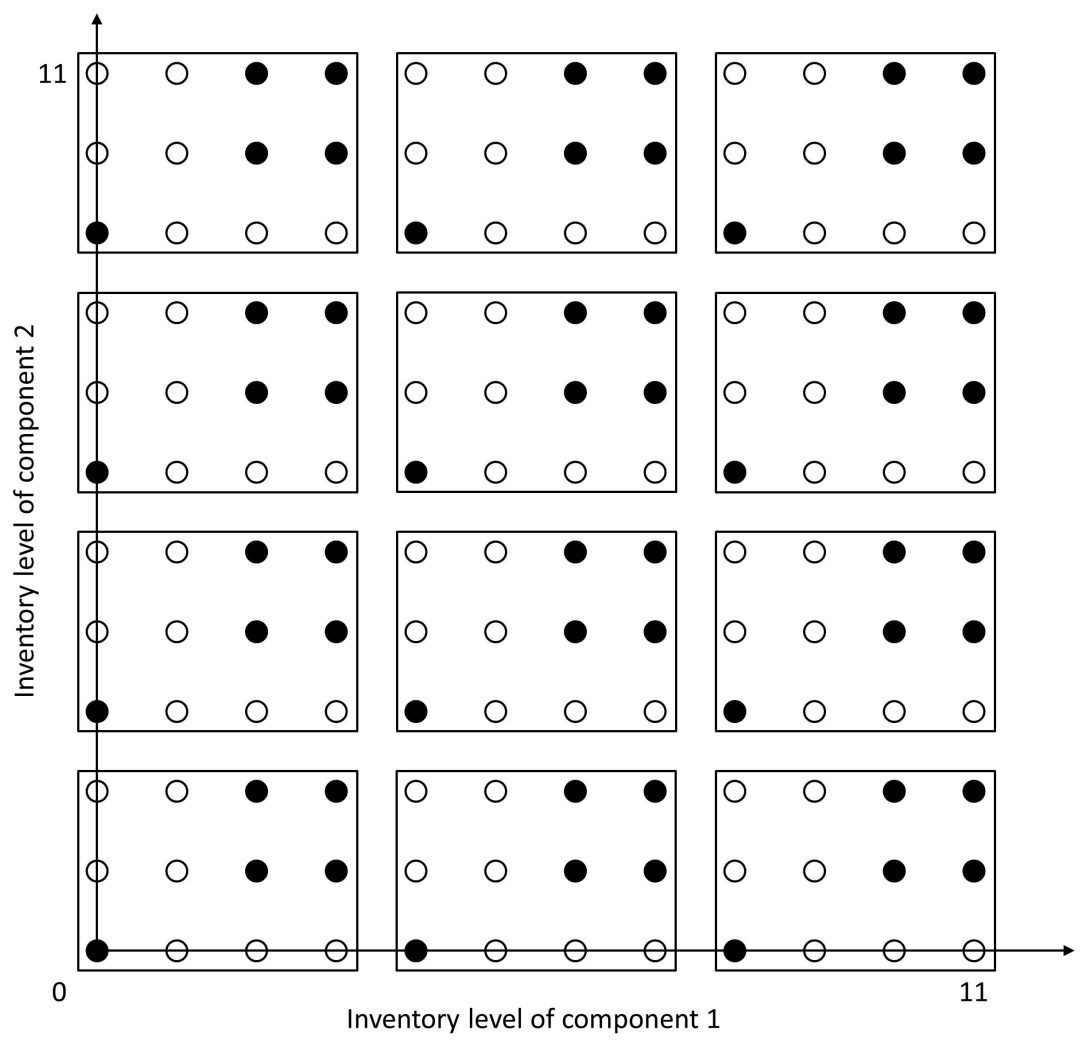

Figure 1 Illustration of our aggregation and disaggregation schemes for a $2 \times 2$ ATO system with $\mathbf{A}=((2,1),(1,1))$ and $\mathbf{q}=(1,1)$, and for the choice $\mathbf{b}=(4,3)$. Each circle (filled or unfilled) forms a different original state. Each rectangle forms a different aggregate state and has $4 \times 3=12$ original states. For instance, the upper right rectangle corresponds to $\mathbf{y}=(2,3)$. Disaggregation probabilities can be positive for original states shown in filled circles, whereas they are zero for other original states, e.g., $d_{(0,0),(2,1)} \geq 0$ and $d_{(0,0),(1,1)}=0$. Also, disaggregation probabilities for original states on the same location of different aggregate states are the same, e.g., $d_{(0,0),(2,1)}=d_{(1,2),(6,7)}$.

is satisfied. Likewise, the system moves from the highest original state of a particular aggregate state to a different aggregate state whenever a batch of any component is produced. Section 6.1 compares the cost performance of this approach, which obeys Definition 2 when $b_{i}>\max _{j} a_{i j}, \forall i$, with that of assigning positive disaggregation probabilities to each original state in each aggregate state. See Figure 2(b) in Section 6.1 for an example of the latter approach. The results obtained tend to confirm our intuition.

Our disaggregation scheme also substantially reduces the per iteration computational complexity of the standard value iteration algorithm that we employ to solve the aggregate problem. Since the original states with zero disaggregation probabilities will never be visited in the aggregate problem, there is no need to execute computations (8)-(10) for those states in the value iteration algorithm. This reduces the per iteration computational complexity of the original problem by a 
factor of $\delta^{-1} \Pi_{i} b_{i}$, where $\delta$ is the number of original states with positive disaggregation probabilities in any aggregate state. For instance, the disaggregation scheme in Figure 1 reduces the per iteration computational complexity by a factor of 2.4. Our numerical experiments reveal that the number of iterations required for convergence of the value iteration algorithm in the aggregate problem is typically only slightly greater than in the original problem. Thus the per iteration computational savings from our disaggregation scheme translate into significant savings in value function computation; see Sections 6.1 and 6.2.

Furthermore, in Section 4 we are able to characterize the structure of the aggregate-optimal policy under our disaggregation scheme. Incorporating our structural results into the value iteration algorithm further alleviates the computational burden of the aggregate problem; see Section 6.3.

\section{Characterization of the Aggregate-Optimal Policy}

We next establish the aggregate-optimal inventory replenishment and allocation policies through the structural properties of our aggregate-optimal cost function. Define $\mathcal{V}$ as the set of real-valued functions $f$ on $\mathbb{N}_{0}^{m}$ that satisfy the following properties:

Property 1. $f\left(\mathbf{y}+\mathbf{e}+e_{i}\right)-f\left(\mathbf{y}+e_{i}\right) \geq f(\mathbf{y}+\mathbf{e})-f(\mathbf{y}), \forall \mathbf{y} \in \mathbb{N}_{0}^{m}$ and $\forall i$,

Property 2. $f\left(\mathbf{y}+e_{i}\right)-f(\mathbf{y}) \geq f\left(\mathbf{y}+e_{i}+e_{k}\right)-f\left(\mathbf{y}+e_{k}\right), \forall \mathbf{y} \in \mathbb{N}_{0}^{m}, \forall i$, and $\forall k \neq i$,

Property 3. $f\left(\mathbf{y}+2 e_{i}\right)-f\left(\mathbf{y}+e_{i}\right) \geq f\left(\mathbf{y}+e_{i}\right)-f(\mathbf{y}), \forall \mathbf{y} \in \mathbb{N}_{0}^{m}$ and $\forall i$.

Property 1 states that the difference $f(\mathbf{y}+\mathbf{e})-f(\mathbf{y})$ is nondecreasing in each of the variables $y_{i}$. Property 2 is equivalent to Topkis' $(1978,1998)$ submodularity property on $\mathbb{N}_{0}^{m}$. Property 3 implies discrete convexity in each of the variables $y_{i}$. It can be shown that Properties 1 and 2 together imply Property 3. Several authors dealing with the optimal policy characterization for Markovian inventory systems prove that the optimal cost function satisfies Properties $1-3$. See, for instance, Benjaafar and ElHafsi (2006), ElHafsi et al. (2008), ElHafsi (2009), and Gayon et al. (2009). However, no one to the best of our knowledge has studied these properties to identify the structure of value function approximations for Markovian inventory systems.

Define the operator $F$ on the set of real-valued functions $r$ :

$$
F r(\mathbf{y})=\sum_{\mathbf{x} \in \mathbf{y}} d_{\mathbf{y x}}\left(h(\mathbf{x})+\sum_{i} \mu_{i} v^{(i)}(\mathbf{x})+\sum_{j} \lambda_{j} v_{j}(\mathbf{x})\right), \quad \mathbf{y} \in \mathcal{Y},
$$

where the function $v^{(i)}$ for component $i$ is given by

$$
v^{(i)}(\mathbf{x})=\min \left\{r\left(\mathbf{y}\left(\mathbf{x}+q_{i} e_{i}\right)\right), r(\mathbf{y}(\mathbf{x}))\right\}, \quad \forall i \in\{1, . ., m\},
$$


and the function $v_{j}$ for product $j$ is given by

$$
v_{j}(\mathbf{x})=\left\{\begin{array}{ll}
\min \left\{r(\mathbf{y}(\mathbf{x}))+c_{j}, r\left(\mathbf{y}\left(\mathbf{x}-\mathbf{a}_{j}\right)\right)\right\} & \text { if } \mathbf{x} \geq \mathbf{a}_{j}, \\
r(\mathbf{y}(\mathbf{x}))+c_{j} & \text { otherwise, }
\end{array} \quad \forall j \in\{1, . ., n\} .\right.
$$

Lemma 1 establishes that $\mathcal{V}$ propagates through the operator $F$, and that our aggregate-optimal cost function is an element of $\mathcal{V}$. (The proofs of Lemma 1 and all other subsequent results appear in the online appendix.)

Lemma 1. Under our aggregation and disaggregation schemes introduced in Definitions 1 and 2, respectively, if $r \in \mathcal{V}$, then $F r \in \mathcal{V}$. Furthermore, the aggregate-optimal cost function $r^{*}$ is an element of $\mathcal{V}$.

Lemma 1 states that our aggregate-optimal cost function satisfies Properties $1-3$. The form of the aggregate-optimal policy in Theorem 1 builds upon Property 1, whereas the comparative statics of the aggregate-optimal policy parameters build upon both Properties 1 and 2 .

It is possible to construct numerical examples showing that Property 1 may fail to hold when our disaggregation scheme is violated. For instance, consider a 1-component 3-product system. Suppose that $\mathbf{A}=((2,1),(1,1),(2,2)), \mathbf{q}=(1,1,1), h_{1}=h_{2}=5, h_{3}=3, c_{1}=100, c_{2}=25, \mu_{1}=\mu_{2}=\mu_{3}=1$, $\lambda_{1}=\lambda_{2}=0.25, \alpha=0.18, \overline{\mathbf{x}}=(11,11,11)$. In addition, suppose that $\mathbf{b}=(3,3,3)$ and $d_{\mathbf{y x}}=1 / 27$, $\forall \mathbf{x} \in \mathbf{y}, \forall \mathbf{y}$. Our disaggregation scheme is violated and Property 1 fails to hold in this instance.

We introduce the notation $\mathbb{L}(\mathbf{p}, \mathbf{b})=\left\{\mathbf{p}+k \mathbf{b}: k \in \mathbb{N}_{0}\right\}$ to denote an $m$-dimensional lattice with initial vector $\mathbf{p} \in \mathbb{N}_{0}^{m}$ and common difference $\mathbf{b}$, where $\exists i$ such that $p_{i}<b_{i}$. Note that, for any $\mathbf{b}$,

$$
\mathbb{N}_{0}^{m}=\bigcup_{\mathbf{p}} \mathbb{L}(\mathbf{p}, \mathbf{b})
$$

and

$$
\mathbb{L}\left(\mathbf{p}_{1}, \mathbf{b}\right) \cap \mathbb{L}\left(\mathbf{p}_{2}, \mathbf{b}\right)=\emptyset, \forall \mathbf{p}_{1}, \mathbf{p}_{2} \text { s.t. } \mathbf{p}_{1} \neq \mathbf{p}_{2}
$$

In other words, we partition the original state space into multiple disjoint lattices with common difference $\mathbf{b}$. This partitioning of the state space implies that each original state in a particular aggregate state belongs to a different lattice.

The structural properties of our aggregate-optimal cost function allow us to characterize the form of the aggregate-optimal policy over such lattices of the original state space:

THEOREM 1. Under our aggregation and disaggregation schemes introduced in Definitions 1 and 2, respectively, there exists an aggregate-optimal stationary policy that can be specified as follows. 
(1) The aggregate-optimal inventory replenishment policy for each component $i$ is a lattice-dependent base-stock policy with lattice-dependent base-stock levels $S_{i}^{*}(\mathbf{p}) \in \mathbb{L}(\mathbf{p}, \mathbf{b}), \forall \mathbf{p}$ : It is aggregate-optimal to produce a batch of component $i$ if and only if $\mathbf{x} \in \mathbb{L}(\mathbf{p}, \mathbf{b})$ is less than $S_{i}^{*}(\mathbf{p})$.

(2) The aggregate-optimal inventory allocation policy for each product $j$ is a lattice-dependent rationing policy with lattice-dependent rationing levels $R_{j}^{*}(\mathbf{p}) \in \mathbb{L}(\mathbf{p}, \mathbf{b}), \forall \mathbf{p}$ : It is aggregateoptimal to fulfill a demand for product $j$ if and only if $\mathbf{x} \in \mathbb{L}(\mathbf{p}, \mathbf{b})$ is greater than or equal to $R_{j}^{*}(\mathbf{p})$.

The aggregate-optimal policy has the following additional properties:

(i) The controller is indifferent between producing and not producing a batch of component $i$ at aggregate-optimality if $\mathbf{x} \in \mathbb{L}(\mathbf{p}, \mathbf{b})$ and $\mathbf{y}\left(\mathbf{p}+q_{i} e_{i}\right)=\mathbf{y}(\mathbf{p})$.

(ii) $\mathbf{y}\left(S_{i}^{*}(\mathbf{p})\right)=\mathbf{y}\left(S_{i}^{*}(\mathbf{r})\right)$ if $\mathbf{y}(\mathbf{p})+e_{i}=\mathbf{y}(\mathbf{r})+e_{i}=\mathbf{y}\left(\mathbf{p}+q_{i} e_{i}\right)=\mathbf{y}\left(\mathbf{r}+q_{i} e_{i}\right), \forall i$.

(iii) $S_{i}^{*}\left(\mathbf{p}+b_{k} e_{k}\right) \geq S_{i}^{*}(\mathbf{p})+b_{k} e_{k}$ if $\mathbf{y}(\mathbf{p})+e_{i}=\mathbf{y}\left(\mathbf{p}+q_{i} e_{i}\right), \forall k \neq i$.

(iv) $S_{i}^{*}(\mathbf{p})+\sum_{k \in I} b_{k} e_{k} \geq S_{i}^{*}\left(\mathbf{p}+\sum_{k \in I} b_{k} e_{k}\right)$ if $\mathbf{y}(\mathbf{p})+e_{i}=\mathbf{y}\left(\mathbf{p}+q_{i} e_{i}\right)$, where $\{i\} \subseteq I \subset\{1, . ., m\}$, $\forall i$.

(v) It is aggregate-optimal to fulfill a demand for product $j$ if $\mathbf{x} \in \mathbb{L}(\mathbf{p}, \mathbf{b})$ where $\mathbf{p} \geq \mathbf{a}_{j}$ and $\mathbf{y}(\mathbf{p}-$ $\left.\mathbf{a}_{j}\right)=\mathbf{y}(\mathbf{p})$.

(vi) $\mathbf{y}\left(R_{j}^{*}(\mathbf{p})\right)=\mathbf{y}\left(R_{j}^{*}(\mathbf{r})\right)$ if $\mathbf{y}(\mathbf{p})=\mathbf{y}(\mathbf{r})=\mathbf{y}\left(\mathbf{p}+\mathbf{b}-\mathbf{a}_{j}\right)=\mathbf{y}\left(\mathbf{r}+\mathbf{b}-\mathbf{a}_{j}\right), \forall j$.

(vii) $R_{j}^{*}(\mathbf{p})+b_{i} e_{i} \geq R_{j}^{*}\left(\mathbf{p}+b_{i} e_{i}\right)$ if $\mathbf{y}\left(\mathbf{p}+\mathbf{b}-\mathbf{a}_{j}\right)=\mathbf{y}(\mathbf{p}), \forall i, j$.

Using Property 1, Theorem 1 shows that a lattice-dependent base-stock and lattice-dependent rationing (LBLR) policy is the aggregate-optimal inventory replenishment and allocation policy under our aggregation and disaggregation schemes: Property 1 implies that, as the system moves to a higher inventory level on the lattice $\mathbb{L}(\mathbf{p}, \mathbf{b})$, the desirability of producing a batch of component $i$ decreases in a non-strict sense (aggregate-optimality of base-stock policies, point 1), and the desirability of satisfying a demand for product $j$ increases in a non-strict sense (aggregate-optimality of rationing policies, point 2).

Theorem 1 establishes the following additional properties of the aggregate-optimal replenishment policy: Point (i) says that if the current aggregate state remains the same upon replenishment of a batch of component $i$, there is no difference between producing and not producing component $i$ at aggregate-optimality. Point (ii) shows that if the system moves from two different points of two disjoint lattices in a particular aggregate state $\mathbf{y}$ to the same aggregate state $\mathbf{y}+e_{i}$ upon 
replenishment of a batch of component $i$, the aggregate-optimal base-stock levels of component $i$ on these two lattices must be in the same aggregate state. Based on Property 2, point (iii) states that, as the system moves to a different lattice with an increment of $b_{k}$ in the inventory level of component $k$, the aggregate-optimal base-stock level of component $i \neq k$ increases in a non-strict sense. However, based on Properties 1 and 2, point (iv) says that, as the system moves to a different lattice with an increment of $b_{k}$ in the inventory level of component $k$ and an increment of $b_{i}$ in the inventory level of component $i \neq k$, the aggregate-optimal base-stock level of component $i$ increases by no more than $b_{k} e_{k}+b_{i} e_{i}$.

As for the aggregate-optimal allocation policy, Theorem 1 establishes the following additional properties: Point (v) shows that it is aggregate-optimal to satisfy a demand for product $j$ if the current aggregate state remains the same upon fulfillment of this demand. Point (vi) states that if the system moves from two different points of two disjoint lattices in a particular aggregate state to a lower aggregate state upon fulfillment of a demand for product $j$, the aggregate-optimal rationing levels for product $j$ on these two lattices must be in the same aggregate state. Based on Property 1 , point (vii) says that, upon fulfillment of a demand for product $j$, if the system moves from one particular lattice in any aggregate state to a lower aggregate state, then the following must hold: As the system moves from this particular lattice to a different lattice with an increment of $b_{i}$ in the inventory level of component $i$, the aggregate-optimal rationing level for product $j$ increases by $b_{i} e_{i}$ or decreases.

The notion of LBLR was first introduced by Nadar et al. (2014) to characterize the optimal policy structure for ATO generalized $M$-systems under Markovian assumptions on production and demand. Nadar et al. (2014) also established the comparative statics of the optimal policy parameters for generalized $M$-systems, which are in line with our results in points (iii) and (vii) of Theorem 1. In their computational work, Nadar et al. (2015) reveal the optimal performance of LBLR for ATO systems with general product structures, but present no method for its proof. Unlike Nadar et al. $(2014,2015)$, we use the notion of LBLR to analytically characterize the aggregateoptimal policy form for ATO systems with general product structures under our aggregation and disaggregation schemes, partially bridging this gap. Also, our partitioning of the state space into disjoint lattices is based on the vector $\mathbf{b}$ that we use to construct our aggregation scheme; that in Nadar et al. $(2014,2015)$ is based on certain assumed product characteristics that we do not require. 
Section 6.3 implements the above structural results into a value iteration algorithm, by restricting the action space to only generate LBLR policies as the algorithm successively visits the original states in each iteration step. This further reduces the computation time of the aggregate problem in our numerical experiments.

\section{Error Bounds for the Aggregate Problem}

We are able to establish, in Theorem 2, the existence of a finite error bound for our aggregateoptimal cost function (not necessarily obeying our disaggregation scheme in Definition 2) under the following mild condition.

Assumption 1. There exists a product (denoted by $j^{*}$ ) such that (i) one unit of this product consumes at least one unit from each component and (ii) it is always optimal to satisfy demands for this product if sufficient inventory exists.

Assumption 1 is satisfied in many ATO systems with different product structures. Below are three specific types of ATO product structures that satisfy Assumption 1, which are special cases of our general ATO problem in Section 2.

(1) An assembly product structure. Suppose that $a_{i j}=1, \forall i, j$. In such ATO systems, Benjaafar and ElHafsi (2006) showed that it is always optimal to satisfy demands for the product with the highest lost sale cost if sufficient inventory is available.

(2) A nested product structure with unitary component requirements. Suppose that $a_{i j}=1$ if $i \geq j$, and $a_{i j}=0$ otherwise, and $c_{1}>. .>c_{m}$. In such ATO systems, ElHafsi et al. (2008) showed that it is always optimal to satisfy demands for product 1 if sufficient inventory is available.

(3) A nested product structure with non-unitary component requirements. Let $\tau_{j}$ denote an arbitrary positive integer, $\forall j$. Suppose that $a_{i 1}=1, \forall i, a_{i j}=\tau_{1} \times . . \times \tau_{j-1}, \forall i, \forall j>1, c_{j+1} \geq \tau_{j} c_{j}$, $\forall j<n$, and $\mathbf{q}=\mathbf{a}_{n}$. Then it is always optimal to satisfy demands for product $n$ if sufficient inventory is available; we provide a proof of this result in the online appendix.

Assumption 1 fails to hold, for example, when there is no product that uses all the components. It is also possible to construct ATO systems showing that Assumption 1 may fail to hold even when there is a product that uses all the components. For instance, consider a 2-component 2-product system. Suppose that $\mathbf{A}=((1,2),(2,1)), \mathbf{q}=(1,1), h_{1}=h_{2}=3, c_{1}=c_{2}=100, \mu_{1}=\mu_{2}=\lambda_{1}=\lambda_{2}=1$, $\alpha=0.25$, and $\overline{\mathbf{x}}=(20,20)$. Then it is optimal to fulfill a demand for product 1 in state $\mathbf{x}=(2,10)$, whereas it is not optimal to do so in state $\mathbf{x}=(10,2)$. The reverse is true for product 2 . 
Under Assumption 1, Lemma 2 establishes upper bounds on the difference of the optimal cost function in (4) evaluated at any two original states.

Lemma 2. Under Assumption 1, the following inequality holds for any two original states $\mathbf{x}_{1}$ and $\mathbf{x}_{2}$.

$$
v^{*}\left(\mathbf{x}_{1}\right)-v^{*}\left(\mathbf{x}_{2}\right) \leq \frac{h\left(\mathbf{x}_{1}-\mathbf{x}_{2}+\tau \mathbf{a}_{j^{*}}\right)}{\alpha}+\tau c_{j^{*}}
$$

where

$$
\tau=\min \left\{k \in \mathbb{N}_{0}: k \mathbf{a}_{j^{*}} \geq \mathbf{x}_{2}-\mathbf{x}_{1}\right\}
$$

Lemma 2 implies that when $\mathbf{x}_{1} \geq \mathbf{x}_{2}, v^{*}\left(\mathbf{x}_{1}\right) \leq v^{*}\left(\mathbf{x}_{2}\right)+h\left(\mathbf{x}_{1}-\mathbf{x}_{2}\right) / \alpha$ (i.e., $\left.\tau=0\right)$ : When the system moves to a higher state, the optimal cost increases by no more than the maximum possible increase in inventory holding costs, which occurs when the additional $x_{1 i}-x_{2 i}$ units of each component $i$ remain in inventory for a very long time. Lemma 2 also implies that when $\mathbf{x}_{2}=\tau \mathbf{a}_{j^{*}}+\mathbf{x}_{1}$, $v^{*}\left(\mathbf{x}_{1}\right) \leq v^{*}\left(\mathbf{x}_{2}\right)+\tau c_{j^{*}}$ : When the system moves to a lower state upon fulfillment of $\tau$ demands for product $j^{*}$, the optimal cost function increases by no more than the loss when those demands are rejected. This is because we assume it is always optimal to satisfy demands for product $j^{*}$ if sufficient inventory exists. If neither $\mathbf{x}_{1} \geq \mathbf{x}_{2}$ nor $\mathbf{x}_{2}=\tau \mathbf{a}_{j^{*}}+\mathbf{x}_{1}$ (for $\tau \in \mathbb{N}_{0}$ ), the upper bounds in Lemma 2 can be obtained by creating an auxiliary state $\mathbf{x}_{3}$ such that $\mathbf{x}_{3} \geq \mathbf{x}_{2}$ and $\mathbf{x}_{3}=\tau \mathbf{a}_{j^{*}}+\mathbf{x}_{1}$, and using the above relationships.

In the ATO literature, several authors have derived upper bounds on the optimal cost function difference between certain states for more restricted versions of our general ATO problem. See, for instance Ha (1997, 2000), Benjaafar and ElHafsi (2006), ElHafsi et al. (2008), and ElHafsi (2009). However, to the best of our knowledge, we are the first to introduce upper bounds on the optimal cost function difference between arbitrary states.

The above upper bounds derived under Assumption 1 allow us to construct a finite error bound for our aggregate-optimal cost function based on certain problem parameters $\left(\alpha, h_{i}, c_{j^{*}}\right.$, and $\left.\mathbf{a}_{j^{*}}\right)$ and the vector $\mathbf{b}$.

THEOREM 2. Under Assumption 1, there exists a finite error bound for our aggregate-optimal cost function that can be specified as follows.

$$
r^{*}(\mathbf{y})-\epsilon \leq v^{*}(\mathbf{x}) \leq r^{*}(\mathbf{y})+\epsilon, \quad \forall \mathbf{y} \in \mathcal{Y}, \quad \mathbf{x} \in \mathbf{y}
$$

where

$$
\epsilon=\max _{\substack{\mathbf{x}_{1}, \mathbf{x}_{2} \\ \text { s.t. } 0 \leq x_{1, i}, x_{2, i}<b_{i}, \forall i}} \frac{h\left(\mathbf{x}_{1}-\mathbf{x}_{2}+\tau \mathbf{a}_{j^{*}}\right)+\alpha \tau c_{j^{*}}}{\alpha^{2}}
$$


and

$$
\tau=\min \left\{k \in \mathbb{N}_{0}: k \mathbf{a}_{j^{*}} \geq \mathbf{x}_{2}-\mathbf{x}_{1}\right\}
$$

Notice that our error bound $\epsilon$ increases with the discount factor $1-\alpha$, the unit holding cost rate $h_{i}$, or the unit lost sale cost $c_{j^{*}}$. Our numerical experiments indicate that $\epsilon$ is typically multiple orders of magnitude larger than the actual errors for the instances in Section 6. Although not yet tight, identifying a finite error bound for our aggregate-optimal cost function enables us to validate the following value iteration algorithm: After solving the aggregate problem very quickly, we start the value iteration algorithm in the original problem from the aggregate-optimal cost function. The existence of a finite error bound guarantees the convergence of this algorithm.

Specifically, define the operator $T$ on the set of real-valued functions $v$ :

$$
T v(\mathbf{x})=h(\mathbf{x})+\sum_{i} \mu_{i} T^{(i)} v(\mathbf{x})+\sum_{j} \lambda_{j} T_{j} v(\mathbf{x})
$$

where the operators $T^{(i)}$ and $T_{j}$ are the same as in (5) and (6). Also, let $T^{1} v=T v$ and $T^{k} v=$ $T\left(T^{k-1} v\right), \forall k>1$. We are now ready to state the following result based on the finite error bounds of the aggregate problem.

Theorem 3. Under Assumption 1, the value iteration algorithm starting with the aggregateoptimal cost function converges to the optimal cost function of the original problem, i.e., if $v(\mathbf{x})=$ $r^{*}(\mathbf{y}(\mathbf{x})), \forall \mathbf{x}$, then $\lim _{k \rightarrow \infty}\left(T^{k} v\right)(\mathbf{x})=v^{*}(\mathbf{x}), \forall \mathbf{x}$.

Our numerical experiments in Section 6.4 demonstrate the usefulness of the value iteration algorithm described in Theorem 3 for general ATO systems.

\section{Numerical Experiments}

In Section 6.1 we numerically investigate the performance of our aggregation scheme in Definition 1, when used with one possible disaggregation scheme satisfying Definition 2, as an approximation to the optimal cost function of the original problem. We also compare our disaggregation scheme to an alternative uniform scheme from the literature that assigns equal disaggregation probabilities to each original state in each aggregate state (thus violating Definition 2). This alternative scheme clearly ignores the product characteristics of the ATO problem. After evaluating the scalability of our aggregate problem (satisfying Definitions 1 and 2) in Section 6.2, we exploit the aggregateoptimal policy structure (Theorem 1) in computation of the aggregate-optimal cost function, and analyze the resulting computational savings, in Section 6.3. Finally, in Section 6.4, we evaluate 


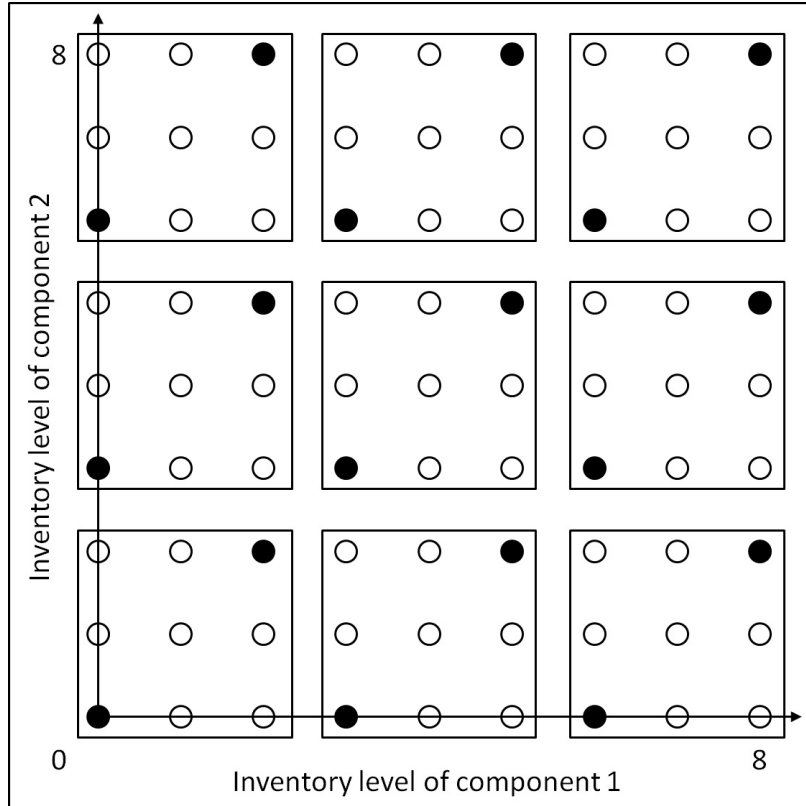

(a)

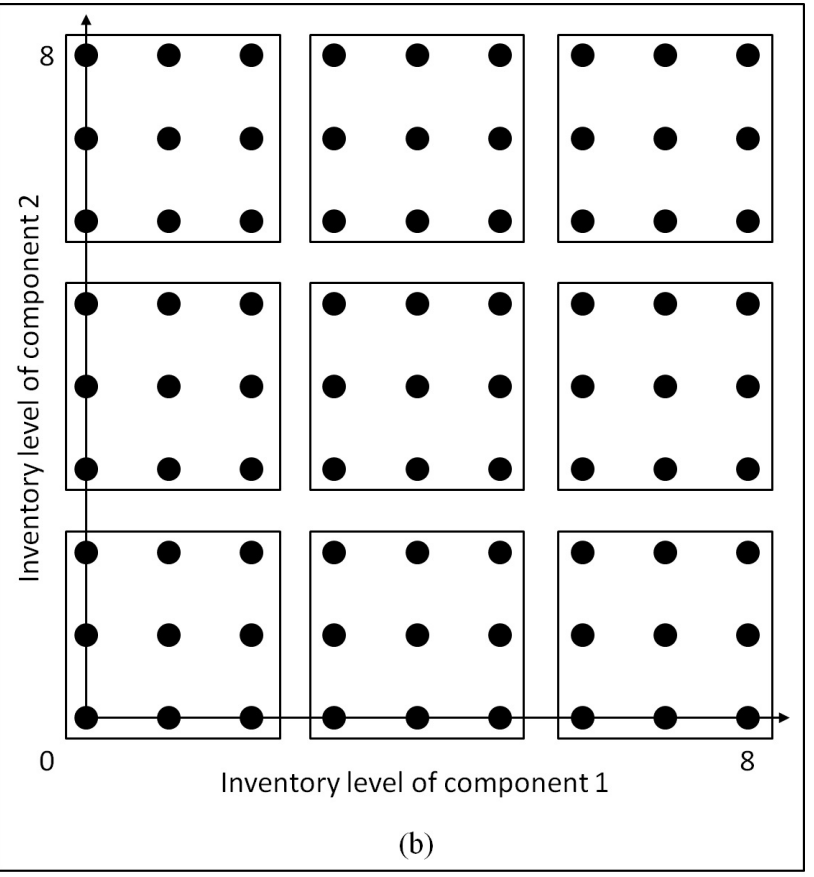

(b)

Figure 2 Illustration of two possible disaggregation schemes for a $2 \times 2$ ATO system and an aggregation scheme with $\mathbf{b}=(3,3)$. Each circle (filled or unfilled) forms a different original state and each square forms a different aggregate state. The disaggregation probability of each original state shown in a filled circle is $1 / 2$ in (a), and is $1 / 9$ in (b).

the use of the value iteration algorithm initialized with the aggregate-optimal cost function in the original problem, comparing it to the standard value iteration algorithm. All computations in this section have been executed on a system with $2.9 \mathrm{GHz}$ CPU and 16 GB of RAM.

\subsection{Performance Evaluation of Fulfillment Based Disaggregation}

In this subsection we investigate the performance of the aggregate-optimal solution, in comparison with the optimal solution of the original problem. We consider the following two disaggregation schemes under our aggregation method in Definition 1:

(1) Extreme point policy: The lowest and highest original states that belong to aggregate state $\mathbf{y}$ are equally representative, i.e.,

$$
d_{\mathbf{y x}}= \begin{cases}1 / 2 & \text { if } x_{i}=b_{i} y_{i} \text { for all } i \\ 1 / 2 & \text { if } x_{i}=b_{i} y_{i}+b_{i}-1 \text { for all } i \\ 0 & \text { otherwise }\end{cases}
$$

(2) Uniform policy: All states that belong to aggregate state $\mathbf{y}$ are equally representative, i.e.,

$$
d_{\mathbf{y x}}= \begin{cases}\left(\prod_{i} b_{i}\right)^{-1} & \text { if } \mathbf{x} \in \mathbf{y} \\ 0 & \text { otherwise }\end{cases}
$$


See Figures 2(a)-(b) for an illustration of cases (1) and (2), respectively. Our fulfillment based disaggregation scheme is satisfied by our extreme point policy when $b_{i}>\max _{j} a_{i j}, \forall i$, while it is violated by the uniform policy. We label the original problem as OP, and the aggregate problems in cases (1) and (2) as AP-Ex and AP-Un, respectively. The acronym "Ex" stands for the extreme points with positive disaggregation probabilities, and "Un" for the uniform disaggregation probabilities across the original states.

Rogers and Plante (1993) evaluate the use of uniform, equal weighting for disaggregation in estimating the limiting probabilities of band diagonal Markov chains. In particular, they compare equal weighting with an alternative technique of assigning the disaggregation weights by solving a group of subsystems in the original problem. Their numerical analysis reveals that the use of equal weighting yields approximate limiting probabilities that are often as good as those obtained from the use of weights associated with the solutions of the subsystems, motivating us to use AP-Un as a natural benchmark for AP-Ex.

We consider 3-component 3-product systems in which $\overline{\mathbf{x}}=(35,35,35)$ and $\nu /(\alpha+\nu) \in$ $\{0.95,0.97,0.99\}(\nu /(\alpha+\nu)$ is the discount factor $)$. For each value of the discount factor, we generate 50 instances by randomly selecting $a_{i j}$ and $q_{i}$ from the set $\{1,2\}, h_{i}$ from the set $\{1,3,5\}$, $c_{j}$ from the set $\{25,50,75,100\}, \mu_{i}$ from the set $\{1,1.5\}$, and $\lambda_{j}$ from the set $\{0.25,0.5\}, \forall i, j$. For AP-Ex and AP-Un, we consider aggregation schemes in which $\mathbf{b} \in\{(3,3,3),(4,4,4),(6,6,6)\}$. (Note that $\bar{x}_{i}+1=36$ is an integer multiple of 3,4 , and 6 , and thus each aggregate state contains the same number of original states under each aggregation scheme. We restricted $a_{i j}$ to be less than 3 in order to implement our extreme point policy, without violating Definition 2, in all instances.) We developed a computer program that uses the value iteration algorithm to compute the optimal cost function in OP, and the aggregate-optimal cost functions in AP-Ex and AP-Un for each of the three aggregation schemes on our test bed of 150 instances. We then evaluate the performance of AP-Ex and AP-Un in terms of the following measures:

(i) OSPD: The Percentage Deviation from the optimal cost evaluated in the Optimal State, i.e.,

$$
100 \times \frac{\left|r^{*}\left(\mathbf{y}\left(\mathbf{x}^{*}\right)\right)-v^{*}\left(\mathbf{x}^{*}\right)\right|}{v^{*}\left(\mathbf{x}^{*}\right)}
$$

where $\mathbf{x}^{*}=\arg \min _{\mathbf{x} \in \mathcal{X}} v^{*}(\mathbf{x})$.

(ii) APD: The Average of Percentage Deviations from the optimal cost across all states, i.e.,

$$
\frac{100}{|\mathcal{X}|} \times \sum_{\mathbf{x} \in \mathcal{X}} \frac{\left|r^{*}(\mathbf{y}(\mathbf{x}))-v^{*}(\mathbf{x})\right|}{v^{*}(\mathbf{x})}
$$

where $|\mathcal{X}|$ is the number of original states in the set $\mathcal{X}$. 
Table 1 Confidence intervals on performance measures for AP-Ex.

\begin{tabular}{|cc|c|c|c|c|}
\hline $\mathbf{b}$ & $\nu /(\alpha+\nu)$ & OSPD & APD & WAPD & CTR \\
\hline$(3,3,3)$ & 0.95 & $8.36 \pm 1.43$ & $5.12 \pm 0.32$ & $15.12 \pm 1.42$ & $0.079 \pm 0.003$ \\
- & 0.97 & $11.78 \pm 1.66$ & $6.23 \pm 0.52$ & $16.57 \pm 1.74$ & $0.079 \pm 0.003$ \\
- & 0.99 & $13.88 \pm 1.71$ & $6.66 \pm 0.65$ & $16.14 \pm 1.81$ & $0.077 \pm 0.004$ \\
$(4,4,4)$ & 0.95 & $16.01 \pm 2.93$ & $6.98 \pm 0.43$ & $12.90 \pm 1.10$ & $0.034 \pm 0.001$ \\
- & 0.97 & $11.08 \pm 3.17$ & $7.48 \pm 0.58$ & $12.10 \pm 1.81$ & $0.035 \pm 0.001$ \\
- & 0.99 & $7.89 \pm 2.31$ & $7.09 \pm 0.66$ & $9.66 \pm 1.79$ & $0.033 \pm 0.001$ \\
$(6,6,6)$ & 0.95 & $34.90 \pm 5.16$ & $12.32 \pm 0.77$ & $18.91 \pm 3.12$ & $0.013 \pm 0.001$ \\
- & 0.97 & $28.38 \pm 4.62$ & $12.35 \pm 0.81$ & $17.20 \pm 3.66$ & $0.012 \pm 0.001$ \\
- & 0.99 & $21.18 \pm 3.66$ & $12.13 \pm 1.23$ & $16.65 \pm 3.54$ & $0.012 \pm 0.001$ \\
\hline \multicolumn{2}{|c|}{ Average } & 17.05 & 8.49 & 15.03 & 0.042 \\
\hline
\end{tabular}

Table 2 Confidence intervals on performance measures for AP-Un.

\begin{tabular}{|cc|c|c|c|c|}
\hline $\mathbf{b}$ & $\nu /(\alpha+\nu)$ & OSPD & APD & WAPD & CTR \\
\hline$(3,3,3)$ & 0.95 & $23.32 \pm 1.64$ & $4.85 \pm 0.28$ & $17.79 \pm 1.55$ & $1.040 \pm 0.034$ \\
- & 0.97 & $19.82 \pm 1.85$ & $5.33 \pm 0.23$ & $16.58 \pm 1.90$ & $1.088 \pm 0.075$ \\
- & 0.99 & $17.55 \pm 1.86$ & $8.29 \pm 0.58$ & $16.05 \pm 2.03$ & $0.977 \pm 0.038$ \\
$(4,4,4)$ & 0.95 & $31.63 \pm 1.51$ & $6.71 \pm 0.41$ & $22.15 \pm 1.91$ & $1.027 \pm 0.035$ \\
- & 0.97 & $27.53 \pm 2.03$ & $7.53 \pm 0.31$ & $20.40 \pm 2.13$ & $1.032 \pm 0.029$ \\
- & 0.99 & $23.00 \pm 2.14$ & $11.57 \pm 0.81$ & $20.16 \pm 2.30$ & $0.975 \pm 0.035$ \\
$(6,6,6)$ & 0.95 & $39.84 \pm 2.81$ & $9.49 \pm 0.58$ & $25.28 \pm 2.67$ & $1.043 \pm 0.031$ \\
- & 0.97 & $32.10 \pm 2.60$ & $10.69 \pm 0.43$ & $23.41 \pm 2.73$ & $1.042 \pm 0.036$ \\
- & 0.99 & $26.84 \pm 2.70$ & $15.81 \pm 1.12$ & $23.20 \pm 2.89$ & $1.014 \pm 0.051$ \\
\hline \multicolumn{2}{|c|}{ Average } & 26.85 & 8.92 & 20.56 & 1.026 \\
\hline
\end{tabular}

(iii) WAPD: The Weighted Average of Percentage Deviations from the optimal cost across all states based on the optimal stationary distribution, i.e.,

$$
100 \times \sum_{\mathbf{x} \in \mathcal{X}} \frac{\pi_{\mathbf{x}}\left|r^{*}(\mathbf{y}(\mathbf{x}))-v^{*}(\mathbf{x})\right|}{v^{*}(\mathbf{x})}
$$

where $\pi_{\mathbf{x}}$ is the limiting probability the system is in state $\mathbf{x}$ at the optimal solution to OP.

(iv) CTR: The Computation Time Ratio of the aggregate problem to the OP.

After calculating each of these performance measures in each instance, we construct $95 \%$ confidence intervals on these measures from our 50 instances with the same discount factor, under each aggregation scheme. Tables 1-2 exhibit our confidence intervals for AP-Ex and AP-Un, respectively.

An important observation from Tables 1-2 is that AP-Ex is computationally much less demanding than AP-Un. As mentioned in Section 3, this is primarily due to the existence of many original states with zero disaggregation probability, and thus fewer original states to evaluate per iteration. 
However, the computational savings from AP-Ex are below our expectations. As the number of states to cover decreases by a factor of $\Pi_{i} b_{i} / 2$ per iteration, we would expect the computation times to be lower than those of OP by the same factor. For instance, when $\mathbf{b}=(6,6,6)$, the number of states to cover in OP is 108 times greater than in AP-Ex. But Table 1 indicates that the computation time of $\mathrm{OP}$ is $0.012^{-1}=83.33$ times greater than that of AP-Ex, on average, when the discount factor is 0.99 . There are several reasons for this result: The aggregate problem (under both disaggregation schemes) typically requires a slightly greater number of iterations until the value iteration algorithm converges. In addition, it involves extra calculation steps per iteration that are required for aggregation in (9)-(10) and disaggregation in (7). These two points also explain the higher computation times of AP-Un than those of OP, on average, in Table 2.

Tables 1-2 also reveal that AP-Ex outperforms AP-Un with respect to each of our performance measures on average: As discussed in Section 3, disaggregating the aggregate state into its two extreme original states better captures the dynamics of replenishment and fulfillment decisions by allowing for more transitions between aggregate states, and thus it can improve the performance of our aggregation scheme.

Table 1 also shows that as expected, APD increases with b. However, at a fixed discount factor, WAPD decreases as $\mathbf{b}$ increases from $(3,3,3)$ to $(4,4,4)$. A possible explanation for this rather contradictory result may be that the aggregate-optimal policy structure does not agree well with the optimal policy structure when $\mathbf{b}=(3,3,3)$. Nadar et al. (2015) reveal the optimal performance of LBLR for the general ATO problem by partitioning the state space into disjoint lattices based on products' component requirements and allowing for different partitioning schemes across components and products. Following the computational results in Nadar et al. (2015), given that $a_{i j} \in\{1,2\}, \forall i, j$, in each of our instances, the state space should be partitioned into lattices with difference vectors including 1 or 2 . Since 4 is an integer multiple of both 1 and 2 , there is a strong probability that the aggregate-optimal policy structure includes the optimal policy structure as a special case when $\mathbf{b}=(4,4,4)$, leading to a better performance than in the case with $\mathbf{b}=(3,3,3)$. (Even if the original and aggregate problems have the same policy structure, the aggregate solution is not necessarily optimal since the policy parameters need not agree.)

We intuitively expect AP-Ex to perform worse at higher discount factors: The original problem becomes more complicated when the future costs get less discounted, and thus it is critical to treat the problem in a sophisticated manner. Our analytical error bounds in Section 5 are in line with this 
Table 3 Numerical results for OP and AP-Ex in selected larger instances.

\begin{tabular}{|c|c|c|c|c|c|c|c|}
\hline \multirow{2}{*}{$(m, n)$} & \multicolumn{2}{|c|}{ Average computation times (in seconds) } & \multicolumn{3}{c|}{ Average APD } \\
\cline { 2 - 8 } & OP & $\mathbf{b}=(3, . ., 3)$ & $\mathbf{b}=(4, . ., 4)$ & $\mathbf{b}=(6, . ., 6)$ & $\mathbf{b}=(3, . ., 3)$ & $\mathbf{b}=(4, . ., 4)$ & $\mathbf{b}=(6, . ., 6)$ \\
\hline$(2,2)$ & 0.751 & 0.172 & 0.111 & 0.065 & 8.47 & 9.47 & 22.55 \\
\hline$(2,3)$ & 0.830 & 0.256 & 0.141 & 0.080 & 11.54 & 13.82 & 25.34 \\
$(2,4)$ & 1.171 & 0.303 & 0.185 & 0.100 & 12.91 & 8.07 & 13.25 \\
$(2,5)$ & 1.511 & 0.360 & 0.217 & 0.115 & 14.64 & 12.59 & 17.03 \\
$(2,6)$ & 1.639 & 0.429 & 0.245 & 0.130 & 18.78 & 14.56 & 14.45 \\
$(2,7)$ & 2.196 & 0.560 & 0.312 & 0.159 & 21.37 & 15.49 & 13.74 \\
$(2,8)$ & 2.097 & 0.479 & 0.286 & 0.144 & 26.43 & 20.69 & 13.61 \\
$(2,9)$ & 2.362 & 0.523 & 0.300 & 0.151 & 29.43 & 23.78 & 14.91 \\
$(2,10)$ & 2.622 & 0.586 & 0.339 & 0.168 & 35.83 & 30.99 & 23.30 \\
\hline$(3,2)$ & 19.037 & 1.490 & 0.679 & 0.263 & 10.22 & 13.61 & 24.42 \\
$(4,2)$ & 589.615 & 18.878 & 8.778 & 4.688 & 8.97 & 10.96 & 18.75 \\
$(5,2)$ & 17496.598 & 239.529 & 130.150 & 91.562 & 7.51 & 9.51 & 16.79 \\
\hline
\end{tabular}

intuition. But, contrary to our expectations, we observe from Table 1 that increasing the discount factor from 0.95 to 0.99 reduces OSPD by 8.12 and WAPD by 3.24 when $\mathbf{b}=(4,4,4)$, and OSPD by 13.72 and WAPD by 2.26 when $\mathbf{b}=(6,6,6)$, on average. This could be because the optimal cost gets much larger as the discount factor grows, and thus even if the aggregate-optimal cost function deviates more from the optimal cost function, its percentage deviation can still decrease.

\subsection{Selected Larger Instances}

To evaluate the scalability of AP-Ex, we construct several instances of various sizes and analyze the computation times of OP and AP-Ex for these instances. We consider systems in which $\overline{\mathbf{x}}=$ $(23, . ., 23)$ and $\nu /(\alpha+\nu)=0.99$, and aggregation schemes in which $\mathbf{b} \in\{(3, . ., 3),(4, . ., 4),(6, . ., 6)\}$. We then generate ten instances of $m$-component $n$-product systems by randomly selecting problem parameters as in Section 6.1. We generate such instances for $m=2$ and $n \in\{2, . ., 10\}$, and for $n=2$ and $m \in\{2,3,4,5\}$. We solve each instance in OP, and in AP-Ex under each aggregation scheme. (We could solve instances with up to 26 products when $m=2$; our computer program runs out of memory when there are more products. We could also solve instances with up to 5 components when $n=2$, in OP, within 5 hours.) Table 3 exhibits the average computation times of $\mathrm{OP}$ and AP-Ex, and the average APD values for AP-Ex in each of the $m$-component $n$-product systems.

Table 3 indicates a close-to-linear increase in the computation times of both OP and AP-Ex with the number of products, with a much lower slope in AP-Ex than in OP. This is because an increment in the number of products has the effect of increasing the number of allocation operators 
in a given state by one in both OP and AP-Ex. Table 3 also shows an exponential increase in the computation times of both OP and AP-Ex with the number of components, with a much lower growth rate in AP-Ex than in OP. A possible explanation for this result is that an increment in the number of components leads to an exponential increase in the state space of OP at a growth rate of $\bar{x}_{i}+1$, and in the state space of AP-Ex at a growth rate of $\left(\bar{x}_{i}+1\right) / b_{i}$. Last, Table 3 indicates that APD tends to increase with the number of products, but decreases with the number of components, on average, under each aggregation scheme.

Our experimental setup is similar to the one proposed by Nadar et al. (2015), who develop a linear program to find the optimal average cost for Markovian ATO systems. Taking $\overline{\mathbf{x}}=(10, . ., 10)$, they could solve instances with up to 15 products when $m=2$, and instances with up to 4 components when $n=2$, within 5 hours. With the help of our aggregation method, given the same time limit, we are able to solve significantly larger instances with much larger bounds on component inventory levels, i.e., much larger state space.

\subsection{The Value of Implementing the Aggregate-Optimal Policy Structure}

Our structural results in Section 4 can be exploited in order to further reduce computation for AP-Ex: The value iteration (VI) algorithm employed in Section 6.1 to solve AP-Ex starts with zero function $r_{0}$ and successively computes $F r_{0}, F^{2} r_{0}, \ldots$, where $F^{k} r_{0}$ refers to $k$ compositions of the operator $F$ defined in Section 4. As $r_{0}$ satisfies Properties $1-3, F^{k} r_{0}$ satisfies Properties 1-3 for all $k$, cf. Lemma 1 . Thus the policy that attains the minimum in each iteration $k$ takes the form of LBLR. As the algorithm progresses in the $k$ th iteration by computing $F^{k} r_{0}$ across states, extra information on the lattice-dependent base-stock and rationing levels becomes available. For instance, in a given iteration, if it is not optimal to produce a batch of component $i$ in state $\mathbf{x} \in \mathbb{L}(\mathbf{p}, \mathbf{b})$, then the base-stock level for component $i$ on the lattice $\mathbb{L}(\mathbf{p}, \mathbf{b})$ should be no greater than $\mathbf{x}$. Now consider states $\mathbf{z} \in \mathbb{L}(\mathbf{p}, \mathbf{b})$ that are greater than $\mathbf{x}$ and have not been visited yet in the current iteration. The action of producing a batch of component $i$ can be discarded from the action space in such states, in order to reduce computation, without loss of optimality.

We therefore modify the VI algorithm as follows. Once the optimal actions are identified in any state in any iteration, we eliminate any action that is inconsistent with LBLR from the action space of those states that will be visited in the current iteration. We also consider a variant of this modified VI algorithm where we eliminate actions that are inconsistent with not only LBLR but also properties (i)-(vii) in Theorem 1. We label these two algorithms as LBLR-VI and LBLRI-VI, 
Table 4 Confidence intervals on computation time ratios of LBLR-VI and LBLRI-VI to VI, respectively.

\begin{tabular}{|ccc|c|c|}
\hline $\mathbf{b}$ & Avg. lattice length & $\nu /(\alpha+\nu)$ & LBLR-VI/VI & LBLRI-VI/VI \\
\hline$(3,3,3)$ & 4.35 & 0.95 & $0.641 \pm 0.013$ & $0.537 \pm 0.008$ \\
- & - & 0.97 & $0.645 \pm 0.011$ & $0.539 \pm 0.008$ \\
- & - & 0.99 & $0.622 \pm 0.016$ & $0.521 \pm 0.015$ \\
$(4,4,4)$ & 3.36 & 0.95 & $0.687 \pm 0.021$ & $0.576 \pm 0.020$ \\
- & - & 0.97 & $0.696 \pm 0.010$ & $0.581 \pm 0.008$ \\
- & - & 0.99 & $0.653 \pm 0.021$ & $0.540 \pm 0.019$ \\
$(6,6,6)$ & 2.37 & 0.95 & $0.788 \pm 0.021$ & $0.684 \pm 0.019$ \\
- & - & 0.97 & $0.698 \pm 0.016$ & $0.587 \pm 0.016$ \\
- & - & 0.99 & $0.641 \pm 0.025$ & $0.533 \pm 0.022$ \\
\hline \multicolumn{5}{r}{} \\
\hline
\end{tabular}

respectively, because LBLR-VI eliminates actions based only on the optimality of threshold policies on each single lattice whereas LBLRI-VI extends the action elimination procedure by incorporating the Interdependent thresholds across different lattices.

We solve each of the 150 compiled instances in Section 6.1 using each of the VI, LBLR-VI, and LBLRI-VI algorithms. Again, we consider the aggregation schemes in Section 6.1. We calculate the ratios of computation times of LBLR-VI and LBLRI-VI to that of VI for each instance under each aggregation scheme. Table 4 exhibits $95 \%$ confidence intervals on computation time ratios for LBLR-VI and LBLRI-VI. Notice that LBLRI-VI performs no worse than LBLR-VI with respect to computation times. Also, for AP-Ex, all of the VI, LBLR-VI, and LBLRI-VI algorithms yield the same cost function in each iteration, and thus the total number of iterations remains the same across all these algorithms. Specifically, LBLRI-VI reduces the computation time of VI by $(100-56.6) \%=43.4 \%$ on average. If we combine the computational savings from AP-Ex (when solved with VI) in Section 6.1 with the additional savings of LBLRI-VI over VI in each instance, LBLRI-VI reduces the computation time of OP by $97.7 \%$ on average.

We note from Table 4 that, as $\mathbf{b}$ decreases at a fixed discount factor, LBLR-VI becomes computationally more desirable (there is one exception when the discount factor is 0.99 ). The increasing benefit of LBLR-VI comes from the fact that as aggregate sets get smaller, the average lattice length increases (also shown in Table 4): This growing number of states along any lattice provides more opportunities for action elimination. We also observe that the greatest computational savings from LBLR-VI always occur when the discount factor is 0.99. Finally, the additional computational savings from LBLRI-VI (with respect to LBLR-VI) are similar across different scenarios. 
Table 5 Average iteration number ratios of AP-VI to Z-VI for

\begin{tabular}{|cc|c|c|c|}
\hline $\mathbf{b}$ & $\nu /(\alpha+\nu)$ & MAXPD $=10$ & MAXPD $=5$ & MAXPD $=1$ \\
\hline$(3,3,3)$ & 0.95 & 0.373 & 0.515 & 0.694 \\
- & 0.97 & 0.394 & 0.544 & 0.706 \\
- & 0.99 & 0.332 & 0.488 & 0.671 \\
$(4,4,4)$ & 0.95 & 0.290 & 0.406 & 0.574 \\
- & 0.97 & 0.273 & 0.407 & 0.586 \\
- & 0.99 & 0.181 & 0.310 & 0.494 \\
$(6,6,6)$ & 0.95 & 0.478 & 0.544 & 0.656 \\
- & 0.97 & 0.418 & 0.503 & 0.628 \\
- & 0.99 & 0.323 & 0.419 & 0.562 \\
\hline \multicolumn{2}{|c|}{ Average } & 0.340 & 0.460 & 0.619 \\
\hline
\end{tabular}

\subsection{Initialization of the Value Iteration Algorithm via the Aggregate Solution}

We now evaluate the performance of the value iteration algorithm on the original problem, comparing initializing it with the aggregate-optimal cost function of AP-Ex versus the standard value iteration algorithm initialized with the zero function. Once again, we consider the 150 compiled instances and the three aggregation schemes in Section 6.1. For each instance and each aggregation scheme, we perform the following experiments:

(i) We solve AP-Ex by employing the LBLRI-VI algorithm in Section 6.3, and denote the computation time by $t$.

(ii) We run the value iteration algorithm that starts with the zero function in OP, until time $t$.

(iii) Denote the value iteration algorithm in OP that starts with the aggregate-optimal cost function obtained in (i) by AP-VI. We run AP-VI until the maximum percentage deviation from the optimal cost across all states (MAXPD) reaches $D$. Let $I_{1}$ denote the number of iterations performed by AP-VI.

(iv) Denote the value iteration algorithm in OP that starts with the cost function obtained at time $t$ in (ii) by Z-VI. We run Z-VI until MAXPD again reaches $D$. Let $I_{2}$ denote the number of iterations performed by Z-VI.

We then take the average of $I_{1} / I_{2}$ from each sample of the 50 instances with the same discount factor, under each aggregation scheme for $D \in\{1,5,10\}$; see Table 5 .

It is apparent from Table 5 that AP-VI is computationally more attractive than Z-VI in all cases. Table 5 also indicates that the average iteration ratio increases as MAXPD decreases. Thus AP-VI is less beneficial percentage-wise when a more accurate cost function is desired. However, 
AP-VI can still reduce the number of iterations by $38 \%$, on average, even when MAXPD is 1 . Another important observation is that AP-VI has the greatest computational benefit over Z-VI when $\mathbf{b}=(4,4,4)$ : As discussed in Section 6.1 , we strongly believe that the aggregate-optimal cost function of AP-Ex when $\mathbf{b}=(4,4,4)$ (or $\mathbf{b}=(6,6,6))$ shares similar structural properties with the optimal cost function on our test bed. But, when $\mathbf{b}=(4,4,4)$, Section 6.1 also reveals that AP-Ex provides a better approximation than in the case with $\mathbf{b}=(6,6,6)$. As a result, the nearoptimal cost function (with varying degrees of accuracy) can be found more easily in AP-VI when $\mathbf{b}=(4,4,4)$. Last, we note from Table 5 that AP-VI provides the greatest computational savings when the discount factor is 0.99 , i.e., when the number of iterations required for convergence of the algorithm is largest.

\section{Concluding Remarks}

We have studied aggregation for value function approximation applied to ATO systems with general product structures, basing our aggregation and disaggregation schemes on the knowledge of key component and product characteristics. The aggregate model under this construction provides significant computational savings over the original problem while still maintaining satisfactory accuracy: When disaggregation probabilities are positive in only two extreme original states, the computation time of the aggregate problem is on average $95.8 \%$ lower than that of the original problem, and the average distance of the aggregate solution from the optimal solution is $8.49 \%$ in our instances of the 3-component 3-product systems. For 5-component 2-product systems the computation times of the aggregate problem are also significantly shorter - by up to three orders of magnitude - than those of the original problem, and the average distance from the optimal solution is $11.27 \%$. However, when we assign uniform disaggregation probabilities to all states by ignoring the problem parameters, we are unable to exploit the potential benefits of aggregation: The average computation time of the aggregate problem is no less than that of the original problem, and there is a greater loss of accuracy relative to our disaggregation scheme.

We also show the optimality of the LBLR policy for the aggregate problem under our disaggregation scheme. By incorporating our structural results into the value iteration algorithm that we employ to solve the aggregate problem, we further reduce the computation time under our disaggregation scheme by $43.4 \%$ on average. In addition, we establish a finite error bound for the aggregate problem when there exists a "dominant product" - one using all the components and for which it is always optimal to satisfy demands if sufficient inventory is available. We use these 
bounds to prove that the value iteration algorithm that we employ to solve the original problem converges to the optimal solution if it starts with the aggregate solution. Numerical experiments in the original problem reveal that the numbers of iterations performed until the maximum distance from the optimal cost across all states drops to $5 \%$ and $1 \%$ are on average $54 \%$ and $38 \%$ lower, respectively, in the value iteration algorithm initialized with the aggregate solution, in comparison with the standard value iteration algorithm.

The structural knowledge that we have shown to be available from the aggregate problem may inspire and facilitate future research on characterizing the optimal policy form and/or developing near-optimal heuristic policies in the original problem for general ATO systems. Future extensions of the current paper could also focus on large-scale computational methods for the aggregate problem, such as simulation-based policy iteration and value iteration methods, possibly at the expense of accuracy. Another direction for future research is to study aggregation in more complex ATO problems that can still be modeled as MDPs with potentially larger state and/or action spaces. Examples include ATO systems with backorders, ATO systems with phase-type component replenishment and/or demand interarrival times, and ATO systems with variable replenishment quantities. Incorporating the component and product characteristics into aggregation and disaggregation schemes might still be very useful in handling such complex problems.

\section{References}

Akçay, Y., S. H. Xu. 2004. Joint inventory replenishment and component allocation optimization in an assemble-to-order system. Management Sci. 50 99-116.

Benjaafar, S., M. ElHafsi. 2006. Production and inventory control of a single product assemble-to-order system with multiple customer classes. Management Sci. 52 1896-1912.

Benjaafar, S., M. ElHafsi, C. Y. Lee, W. Zhou. 2011. Optimal control of an assembly system with multiple stages and multiple demand classes. Oper. Res. 59 522-529.

Bernstein, F., G. DeCroix, Y. Wang. 2011. The impact of demand aggregation through delayed component allocation in an assemble-to-order system. Management Sci. 57 1154-1171.

Bertsekas, D. P. 2012. Dynamic Programming and Optimal Control, Vol. 2. Athena Scientific, Nashua, NH.

Busic, A., I. Vliegen, A. Scheller-Wolf. 2012. Comparing Markov chains: Aggregation and precedence relations applied to sets of states, with applications to assemble-to-order systems. Math. Oper. Res. 37 259-287.

Busic, A., E. Coupechoux. 2014. Exact simulation for assemble-to-order systems. Working paper.

Doğru, M. K., M. I. Reiman, Q. Wang. 2010. A stochastic programming based inventory policy for assembleto-order systems with application to the W model. Oper. Res. 58 849-864. 
Doğru, M. K., M. I. Reiman, Q. Wang. 2014. Stochastic programming based inventory control for the $M$ system. Working paper.

ElHafsi, M., H. Camus, E. Craye. 2008. Optimal control of a nested-multiple-product assemble-to-order system. Internat. J. Production Res. 46 5367-5392.

ElHafsi, M. 2009. Optimal integrated production and inventory control of an assemble-to-order system with multiple non-unitary demand classes. Eur. J. Oper. Res. 194 127-142.

Gayon, J. P., S. Benjaafar, F. de Vericourt. 2009. Using imperfect advance demand information in productioninventory systems with multiple customer classes. Manufacturing Service Oper. Management 11128 143.

Ha, A. 1997. Inventory rationing in a make-to-stock production system with several demand classes and lost sales. Management Sci. 43 1093-1103.

Ha, A. 2000. Stock rationing in an $M / E_{k} / 1$ make-to-stock queue. Management Sci. 46 77-87.

Heyman, D. P., M. J. Sobel. 2003. Stochastic Models in Operations Research, Volume II: Stochastic Optimization. Dover Publications, Inc., Mineola, New York.

Huang, K. 2014. Benchmarking non-first-come-first-served component allocation in an assemble-to-order system. Ann. Oper. Res. $223217-237$.

Kapuscinski, R., R. Q. Zhang, P. Carbonneau, R. Moore, B. Reeves. 2004. Inventory decisions in Dell's supply chain. Interfaces 34 191-205.

Kemeny, J. G., J. L. Snell. 1976. Finite Markov Chains. Springer-Verlag, New York.

Lippman, S. 1975. Applying a new device in the optimization of exponential queueing systems. Oper. Res. $23687-710$.

Lu, Y., J. S. Song. 2005. Order-based cost optimization in assemble-to-order systems. Oper. Res. 53 151-169.

Lu, Y., J. S. Song, D. D. Yao. 2005 Backorder minimization in multiproduct assemble-to-order systems. IIE Trans. 37 763-774.

Lu, Y., J. S. Song, Y. Zhao. 2010. No-holdback allocation rules for continuous-time assemble-to-order systems. Oper. Res. 58 691-705.

Lu, L., J. S. Song, H. Zhang. 2014. Optimal and asymptotically optimal policies for assemble-to-order N-and W-systems. Working paper, Graduate School of Business, Columbia University, Durham, NC, USA.

Nadar, E., M. Akan, A. Scheller-Wolf. 2014. Optimal structural results for assemble-to-order generalized M-systems. Oper. Res. $62571-579$.

Nadar, E., M. Akan, A. Scheller-Wolf. 2015. Experimental results indicating lattice-dependent policies may be optimal for general assemble-to-order systems. Working paper. 
Powell, W. B. 2011. Approximate Dynamic Programming: Solving the Curses of Dimensionality. John Wiley \& Sons, Inc., Hoboken, New Jersey.

Reiman, M. I., Q. Wang. 2012. A stochastic program based lower bound for assemble-to-order inventory systems. Oper. Res. Lett. 40 89-95.

Reiman, M. I., Q. Wang. 2015. Asymptotically optimal inventory control of assemble-to-order systems with identical lead times. Oper. Res. Forthcoming.

Rogers, D. F., R. D. Plante, R. T. Wong, J. R. Evans. 1991. Aggregation and disaggregation techniques and methodology in optimization. Oper. Res. 39 553-582.

Rogers, D. F., R. D. Plante. 1993. Estimating equilibrium probabilities for band diagonal Markov chains using aggregation and disaggregation techniques. Comput. Oper. Res. 20 857-877.

Topkis, D. M. 1978. Minimizing a submodular function on a lattice. Oper. Res. 26 305-321.

Topkis, D. M. 1998. Supermodularity and Complementarity. Princeton University Press, Princeton, NJ.

Tsitsiklis, J. N., B. Van Roy. 1996. Feature-based methods for large scale dynamic programming. Mach. Learn. 22 59-94.

van Jaarsveld, W., T. Dollevoet. 2011. Spare parts inventory control for an aircraft component repair shop. Econometric Institute report EI 2011-24, Erasmus University Rotterdam.

van Jaarsveld, W., A. Scheller-Wolf. 2015. Optimization of industrial-scale assemble-to-order systems. INFORMS J. Comput. Forthcoming.

Vliegen, I. M. H., G. J. van Houtum. 2009. Approximate evaluation of order fill rates for an inventory system of service tools. Int. J. Prod. Econ. 118 339-351.

Wan, H., Q. Wang. 2015. Asymptotically-optimal component allocation for assemble-to-order productioninventory systems. Oper. Res. Lett. Forthcoming.

Xu, P. J., R. Allgor, S. C. Graves. 2009. Benefits of reevaluating real-time order fulfillment decisions. Manufacturing Service Oper. Management 11 340-355.

Zhao, Y., D. Simchi-Levi. 2006. Performance analysis and evaluation of assemble-to-order systems with stochastic sequential lead times. Oper. Res. 54 706-724. 


\section{Online Technical Appendix}

\section{EC.1. Proofs of the Results in Section 4}

Proof of Lemma 1. Define the operator $F$ on the set of real-valued functions $r$ :

$$
\begin{aligned}
\operatorname{Fr}(\mathbf{y}) & =\sum_{\mathbf{x} \in \mathbf{y}} d_{\mathbf{y} \mathbf{x}} v(\mathbf{x}), \quad \mathbf{y} \in \mathcal{Y}, \\
v(\mathbf{x}) & =h(\mathbf{x})+\sum_{i} \mu_{i} v^{(i)}(\mathbf{x})+\sum_{j} \lambda_{j} v_{j}(\mathbf{x}), \\
v^{(i)}(\mathbf{x}) & =\min \left\{r\left(\mathbf{y}\left(\mathbf{x}+q_{i} e_{i}\right)\right), r(\mathbf{y}(\mathbf{x}))\right\}, \quad \forall i \in\{1, . ., m\}, \\
v_{j}(\mathbf{x}) & =\left\{\begin{array}{ll}
\min \left\{r(\mathbf{y}(\mathbf{x}))+c_{j}, r\left(\mathbf{y}\left(\mathbf{x}-\mathbf{a}_{j}\right)\right)\right\} & \text { if } \mathbf{x} \geq \mathbf{a}_{j}, \\
r(\mathbf{y}(\mathbf{x}))+c_{j} & \text { otherwise, }
\end{array} \quad \forall j \in\{1, . ., n\} .\right.
\end{aligned}
$$

In our aggregation scheme, each original state $\mathbf{x}=\left(x_{1}, . ., x_{m}\right)$ that belongs to aggregate state $\mathbf{y}=$ $\left(y_{1}, . ., y_{m}\right)$ can be formulated as $x_{i}=b_{i} y_{i}+z_{i}$ where $z_{i} \in\left\{0,1, . ., b_{i}-1\right\}, \forall i$. Notice that each original state $\mathbf{x} \in \mathbf{y}$ corresponds to a different vector $\mathbf{z}=\left(z_{1}, . ., z_{m}\right)$. (Recall that $b_{i} \geq \max \left\{q_{i}, \max _{j}\left\{a_{i j}\right\}\right\}$, $\forall i$.) Also notice that, under our disaggregation scheme, the operator $F$ can be rewritten as $\operatorname{Fr}(\mathbf{y})=$ $\sum_{\mathbf{z}} d_{\mathbf{z}} v(\mathbf{x})$ where $x_{i}=b_{i} y_{i}+z_{i}, z_{i} \in\left\{0,1, . ., b_{i}-1\right\}, \forall i$, and $d_{\mathbf{z}}=0$ if (1) $\exists i$ s.t. $\min _{j}\left\{a_{i j}\right\} \leq z_{i}<$ $\max _{j}\left\{a_{i j}\right\}$, or (2) $\exists i, k$ s.t. $z_{i}<\min _{j}\left\{a_{i j}\right\}$ and $\max _{j}\left\{a_{k j}\right\} \leq z_{k}$. Since $d_{\mathbf{z}}$ can be positive only for original states $\mathbf{x}$ such that either $z_{i} \geq \max _{j}\left\{a_{i j}\right\}, \forall i$, or $z_{i}<\min _{j}\left\{a_{i j}\right\}, \forall i$, we restrict ourselves to such original states in the remainder of the proof.

We will prove if the function $r$ satisfies Properties 1 and 2, the function $v$ satisfies the following properties for all original states $\mathbf{x}$ such that either $z_{i} \geq \max _{j}\left\{a_{i j}\right\}, \forall i$, or $z_{i}<\min _{j}\left\{a_{i j}\right\}, \forall i$.

$$
\begin{aligned}
& f\left(\mathbf{x}+\mathbf{b}+b_{i} e_{i}\right)-f\left(\mathbf{x}+b_{i} e_{i}\right) \geq f(\mathbf{x}+\mathbf{b})-f(\mathbf{x}), \forall i, \\
& f\left(\mathbf{x}+b_{i} e_{i}\right)-f(\mathbf{x}) \geq f\left(\mathbf{x}+b_{i} e_{i}+b_{k} e_{k}\right)-f\left(\mathbf{x}+b_{k} e_{k}\right), \forall i \text { and } \forall k \neq i .
\end{aligned}
$$

If the function $v$ satisfies Properties (EC.1) and (EC.2), then $F r$ satisfies Properties 1 and 2: Since $x_{i}=b_{i} y_{i}+z_{i}, \forall i, \operatorname{Fr}\left(\mathbf{y}+\mathbf{e}+e_{i}\right)-\operatorname{Fr}\left(\mathbf{y}+e_{i}\right)-\operatorname{Fr}(\mathbf{y}+\mathbf{e})+$ $F r(\mathbf{y})=\sum_{\mathbf{z}} d_{\mathbf{z}}\left(v\left(\mathbf{x}+\mathbf{b}+b_{i} e_{i}\right)-v\left(\mathbf{x}+b_{i} e_{i}\right)-v(\mathbf{x}+\mathbf{b})+v(\mathbf{x})\right) \geq 0$, i.e., $F r$ satisfies Property 1. Again since $x_{i}=b_{i} y_{i}+z_{i}, \forall i, \operatorname{Fr}\left(\mathbf{y}+e_{i}\right)-\operatorname{Fr}(\mathbf{y})-\operatorname{Fr}\left(\mathbf{y}+e_{i}+e_{k}\right)+\operatorname{Fr}\left(\mathbf{y}+e_{k}\right)=$ $\sum_{\mathbf{z}} d_{\mathbf{z}}\left(v\left(\mathbf{x}+b_{i} e_{i}\right)-v(\mathbf{x})-v\left(\mathbf{x}+b_{i} e_{i}+b_{k} e_{k}\right)+v\left(\mathbf{x}+b_{k} e_{k}\right)\right) \geq 0$, i.e., $F r$ satisfies Property 2.

We proceed to show if $r$ satisfies Properties 1 and 2, then the function $v$ satisfies Properties (EC.1) and (EC.2). To this end, first, we will prove the function $h$ satisfies Properties (EC.1) and (EC.2). Then, we will prove if $r$ satisfies Properties 1 and 2, the functions $v^{(i)}$ and $v_{j}$ satisfy Properties (EC.1) and (EC.2), $\forall i, j$.

(a) We below show $h$ satisfies Properties (EC.1) and (EC.2): 
- First we prove $h$ satisfies Property (EC.1): $h\left(\mathbf{x}+\mathbf{b}+b_{i} e_{i}\right)-h\left(\mathbf{x}+b_{i} e_{i}\right)=\sum_{i} h_{i}\left(x_{i}+b_{i}\right)+h_{i} b_{i}-$ $\sum_{i} h_{i} x_{i}-h_{i} b_{i}=\sum_{i} h_{i}\left(x_{i}+b_{i}\right)-\sum_{i} h_{i} x_{i}=h(\mathbf{x}+\mathbf{b})-h(\mathbf{x})$.

- Second we prove $h$ satisfies Property (EC.2): $h\left(\mathbf{x}+b_{i} e_{i}\right)-h(\mathbf{x})=\sum_{i} h_{i} x_{i}+h_{i} b_{i}-\sum_{i} h_{i} x_{i}=$ $\sum_{i} h_{i} x_{i}+h_{i} b_{i}+h_{k} b_{k}-\sum_{i} h_{i} x_{i}-h_{k} b_{k}=h\left(\mathbf{x}+b_{i} e_{i}+b_{k} e_{k}\right)-h\left(\mathbf{x}+b_{k} e_{k}\right)$.

(b) Assume $r$ satisfies Properties 1 and 2. We will show $v^{(i)}$ satisfies Properties (EC.1) and (EC.2).

- First we show $v^{(i)}$ satisfies Property (EC.1), i.e., $v^{(i)}\left(\mathbf{x}+\mathbf{b}+b_{k} e_{k}\right)-v^{(i)}\left(\mathbf{x}+b_{k} e_{k}\right) \geq v^{(i)}(\mathbf{x}+\mathbf{b})-$ $v^{(i)}(\mathbf{x}), \forall k$. There are four different scenarios we need to consider depending on the optimal actions at $v^{(i)}\left(\mathbf{x}+\mathbf{b}+b_{k} e_{k}\right)$ and $v^{(i)}(\mathbf{x})$ (if this inequality holds under suboptimal actions of $v^{(i)}\left(\mathbf{x}+b_{k} e_{k}\right)$ and/or $v^{(i)}(\mathbf{x}+\mathbf{b})$, it also holds under optimal actions of these operators, and thus we do not enforce the optimal actions at these operators). These four scenarios are as follows:

(1) Suppose that $v^{(i)}\left(\mathbf{x}+\mathbf{b}+b_{k} e_{k}\right)=r\left(\mathbf{y}\left(\mathbf{x}+\mathbf{b}+b_{k} e_{k}\right)\right)$ and $v^{(i)}(\mathbf{x})=r(\mathbf{y}(\mathbf{x}))$. As we assume $r$ satisfies Property 1, the following inequalities hold:

$$
\begin{aligned}
v^{(i)}\left(\mathbf{x}+\mathbf{b}+b_{k} e_{k}\right)-v^{(i)}\left(\mathbf{x}+b_{k} e_{k}\right) & \geq r\left(\mathbf{y}\left(\mathbf{x}+\mathbf{b}+b_{k} e_{k}\right)\right)-r\left(\mathbf{y}\left(\mathbf{x}+b_{k} e_{k}\right)\right) \\
& =r\left(\mathbf{y}+\mathbf{e}+e_{k}\right)-r\left(\mathbf{y}+e_{k}\right) \\
& \geq r(\mathbf{y}+\mathbf{e})-r(\mathbf{y}) \\
& =r(\mathbf{y}(\mathbf{x}+\mathbf{b}))-r(\mathbf{y}(\mathbf{x})) \\
& \geq v^{(i)}(\mathbf{x}+\mathbf{b})-v^{(i)}(\mathbf{x})
\end{aligned}
$$

(2) Suppose that $v^{(i)}\left(\mathbf{x}+\mathbf{b}+b_{k} e_{k}\right)=r\left(\mathbf{y}\left(\mathbf{x}+\mathbf{b}+b_{k} e_{k}\right)\right)$ and $v^{(i)}(\mathbf{x})=r\left(\mathbf{y}\left(\mathbf{x}+q_{i} e_{i}\right)\right)$. Also suppose that $k=i$. Since $q_{i} \leq b_{i}, \forall i$, in our aggregation scheme, we should have either $\mathbf{y}\left(\mathbf{x}+q_{k} e_{k}\right)=\mathbf{y}$ or $\mathbf{y}\left(\mathbf{x}+q_{k} e_{k}\right)=\mathbf{y}+e_{k}$. If $\mathbf{y}\left(\mathbf{x}+q_{k} e_{k}\right)=\mathbf{y}$, as we assume $r$ satisfies Property 1, the following inequalities hold:

$$
\begin{aligned}
v^{(i)}\left(\mathbf{x}+\mathbf{b}+b_{k} e_{k}\right)-v^{(i)}\left(\mathbf{x}+b_{k} e_{k}\right) & \geq r\left(\mathbf{y}\left(\mathbf{x}+\mathbf{b}+b_{k} e_{k}\right)\right)-r\left(\mathbf{y}\left(\mathbf{x}+b_{k} e_{k}\right)\right) \\
& =r\left(\mathbf{y}+\mathbf{e}+e_{k}\right)-r\left(\mathbf{y}+e_{k}\right) \\
& \geq r(\mathbf{y}+\mathbf{e})-r(\mathbf{y}) \\
& =r\left(\mathbf{y}\left(\mathbf{x}+\mathbf{b}+q_{k} e_{k}\right)\right)-r\left(\mathbf{y}\left(\mathbf{x}+q_{k} e_{k}\right)\right) \\
& =r\left(\mathbf{y}\left(\mathbf{x}+\mathbf{b}+q_{i} e_{i}\right)\right)-r\left(\mathbf{y}\left(\mathbf{x}+q_{i} e_{i}\right)\right) \\
& \geq v^{(i)}(\mathbf{x}+\mathbf{b})-v^{(i)}(\mathbf{x})
\end{aligned}
$$


If $\mathbf{y}\left(\mathbf{x}+q_{k} e_{k}\right)=\mathbf{y}+e_{k}$, note that $\mathbf{y}\left(\mathbf{x}+q_{k} e_{k}\right)=\mathbf{y}\left(\mathbf{x}+b_{k} e_{k}\right)$. Thus:

$$
\begin{aligned}
v^{(i)}\left(\mathbf{x}+\mathbf{b}+b_{k} e_{k}\right)-v^{(i)}\left(\mathbf{x}+b_{k} e_{k}\right) & \geq r\left(\mathbf{y}\left(\mathbf{x}+\mathbf{b}+b_{k} e_{k}\right)\right)-r\left(\mathbf{y}\left(\mathbf{x}+b_{k} e_{k}\right)\right) \\
& =r\left(\mathbf{y}+\mathbf{e}+e_{k}\right)-r\left(\mathbf{y}+e_{k}\right) \\
& =r\left(\mathbf{y}\left(\mathbf{x}+\mathbf{b}+q_{k} e_{k}\right)\right)-r\left(\mathbf{y}\left(\mathbf{x}+q_{k} e_{k}\right)\right) \\
& =r\left(\mathbf{y}\left(\mathbf{x}+\mathbf{b}+q_{i} e_{i}\right)\right)-r\left(\mathbf{y}\left(\mathbf{x}+q_{i} e_{i}\right)\right) \\
& \geq v^{(i)}(\mathbf{x}+\mathbf{b})-v^{(i)}(\mathbf{x})
\end{aligned}
$$

Now suppose that $k \neq i$. Recall that we should have either $\mathbf{y}\left(\mathbf{x}+q_{i} e_{i}\right)=\mathbf{y}$ or $\mathbf{y}\left(\mathbf{x}+q_{i} e_{i}\right)=$ $\mathbf{y}+e_{i}$. If $\mathbf{y}\left(\mathbf{x}+q_{i} e_{i}\right)=\mathbf{y}$, as we assume $r$ satisfies Property 1 , the following inequalities hold:

$$
\begin{aligned}
v^{(i)}\left(\mathbf{x}+\mathbf{b}+b_{k} e_{k}\right)-v^{(i)}\left(\mathbf{x}+b_{k} e_{k}\right) & \geq r\left(\mathbf{y}\left(\mathbf{x}+\mathbf{b}+b_{k} e_{k}\right)\right)-r\left(\mathbf{y}\left(\mathbf{x}+b_{k} e_{k}+q_{i} e_{i}\right)\right) \\
& =r\left(\mathbf{y}+\mathbf{e}+e_{k}\right)-r\left(\mathbf{y}+e_{k}\right) \\
& \geq r(\mathbf{y}+\mathbf{e})-r(\mathbf{y}) \\
& =r(\mathbf{y}(\mathbf{x}+\mathbf{b}))-r\left(\mathbf{y}\left(\mathbf{x}+q_{i} e_{i}\right)\right) \\
& \geq v^{(i)}(\mathbf{x}+\mathbf{b})-v^{(i)}(\mathbf{x})
\end{aligned}
$$

If $\mathbf{y}\left(\mathbf{x}+q_{i} e_{i}\right)=\mathbf{y}+e_{i}$, as we assume $r$ satisfies Properties 1 and 2 , the following inequalities hold:

$$
\begin{aligned}
v^{(i)}\left(\mathbf{x}+\mathbf{b}+b_{k} e_{k}\right)-v^{(i)}\left(\mathbf{x}+b_{k} e_{k}\right) & \geq r\left(\mathbf{y}\left(\mathbf{x}+\mathbf{b}+b_{k} e_{k}\right)\right)-r\left(\mathbf{y}\left(\mathbf{x}+b_{k} e_{k}+q_{i} e_{i}\right)\right) \\
& =r\left(\mathbf{y}+\mathbf{e}+e_{k}\right)-r\left(\mathbf{y}+e_{k}+e_{i}\right) \\
& \geq r(\mathbf{y}+\mathbf{e})-r(\mathbf{y})+r\left(\mathbf{y}+e_{k}\right)-r\left(\mathbf{y}+e_{k}+e_{i}\right) \\
& \geq r(\mathbf{y}+\mathbf{e})-r\left(\mathbf{y}+e_{i}\right) \\
& =r(\mathbf{y}(\mathbf{x}+\mathbf{b}))-r\left(\mathbf{y}\left(\mathbf{x}+q_{i} e_{i}\right)\right) \\
& \geq v^{(i)}(\mathbf{x}+\mathbf{b})-v^{(i)}(\mathbf{x})
\end{aligned}
$$

(3) Suppose that $v^{(i)}\left(\mathbf{x}+\mathbf{b}+b_{k} e_{k}\right)=r\left(\mathbf{y}\left(\mathbf{x}+\mathbf{b}+b_{k} e_{k}+q_{i} e_{i}\right)\right)$ and $v^{(i)}(\mathbf{x})=r(\mathbf{y}(\mathbf{x}))$. Again we should have either $\mathbf{y}\left(\mathbf{x}+q_{i} e_{i}\right)=\mathbf{y}$ or $\mathbf{y}\left(\mathbf{x}+q_{i} e_{i}\right)=\mathbf{y}+e_{i}$. If $\mathbf{y}\left(\mathbf{x}+q_{i} e_{i}\right)=\mathbf{y}$, as we assume $r$ satisfies Property 1, the following inequalities hold:

$$
v^{(i)}\left(\mathbf{x}+\mathbf{b}+b_{k} e_{k}\right)-v^{(i)}\left(\mathbf{x}+b_{k} e_{k}\right) \geq r\left(\mathbf{y}\left(\mathbf{x}+\mathbf{b}+b_{k} e_{k}+q_{i} e_{i}\right)\right)-r\left(\mathbf{y}\left(\mathbf{x}+b_{k} e_{k}+q_{i} e_{i}\right)\right)
$$




$$
\begin{aligned}
& =r\left(\mathbf{y}+\mathbf{e}+e_{k}\right)-r\left(\mathbf{y}+e_{k}\right) \\
& \geq r(\mathbf{y}+\mathbf{e})-r(\mathbf{y}) \\
& =r(\mathbf{y}(\mathbf{x}+\mathbf{b}))-r(\mathbf{y}(\mathbf{x})) \\
& \geq v^{(i)}(\mathbf{x}+\mathbf{b})-v^{(i)}(\mathbf{x})
\end{aligned}
$$

If $\mathbf{y}\left(\mathbf{x}+q_{i} e_{i}\right)=\mathbf{y}+e_{i}$, as we assume $r$ satisfies Property 1 , the following inequalities hold:

$$
\begin{aligned}
v^{(i)}\left(\mathbf{x}+\mathbf{b}+b_{k} e_{k}\right)-v^{(i)}\left(\mathbf{x}+b_{k} e_{k}\right) & \geq r\left(\mathbf{y}\left(\mathbf{x}+\mathbf{b}+b_{k} e_{k}+q_{i} e_{i}\right)\right)-r\left(\mathbf{y}\left(\mathbf{x}+b_{k} e_{k}+q_{i} e_{i}\right)\right) \\
& =r\left(\mathbf{y}+\mathbf{e}+e_{k}+e_{i}\right)-r\left(\mathbf{y}+e_{k}+e_{i}\right) \\
& \geq r\left(\mathbf{y}+\mathbf{e}+e_{i}\right)-r\left(\mathbf{y}+e_{i}\right) \\
& \geq r(\mathbf{y}+\mathbf{e})-r(\mathbf{y}) \\
& =r(\mathbf{y}(\mathbf{x}+\mathbf{b}))-r(\mathbf{y}(\mathbf{x})) \\
& \geq v^{(i)}(\mathbf{x}+\mathbf{b})-v^{(i)}(\mathbf{x})
\end{aligned}
$$

(4) Suppose that $v^{(i)}\left(\mathbf{x}+\mathbf{b}+b_{k} e_{k}\right)=r\left(\mathbf{y}\left(\mathbf{x}+\mathbf{b}+b_{k} e_{k}+q_{i} e_{i}\right)\right)$ and $v^{(i)}(\mathbf{x})=r\left(\mathbf{y}\left(\mathbf{x}+q_{i} e_{i}\right)\right)$. Again we should have either $\mathbf{y}\left(\mathbf{x}+q_{i} e_{i}\right)=\mathbf{y}$ or $\mathbf{y}\left(\mathbf{x}+q_{i} e_{i}\right)=\mathbf{y}+e_{i}$. If $\mathbf{y}\left(\mathbf{x}+q_{i} e_{i}\right)=\mathbf{y}$, as we assume $r$ satisfies Property 1, the following inequalities hold:

$$
\begin{aligned}
v^{(i)}\left(\mathbf{x}+\mathbf{b}+b_{k} e_{k}\right)-v^{(i)}\left(\mathbf{x}+b_{k} e_{k}\right) & \geq r\left(\mathbf{y}\left(\mathbf{x}+\mathbf{b}+b_{k} e_{k}+q_{i} e_{i}\right)\right)-r\left(\mathbf{y}\left(\mathbf{x}+b_{k} e_{k}+q_{i} e_{i}\right)\right) \\
& =r\left(\mathbf{y}+\mathbf{e}+e_{k}\right)-r\left(\mathbf{y}+e_{k}\right) \\
& \geq r(\mathbf{y}+\mathbf{e})-r(\mathbf{y}) \\
& =r\left(\mathbf{y}\left(\mathbf{x}+\mathbf{b}+q_{i} e_{i}\right)\right)-r\left(\mathbf{y}\left(\mathbf{x}+q_{i} e_{i}\right)\right) \\
& \geq v^{(i)}(\mathbf{x}+\mathbf{b})-v^{(i)}(\mathbf{x})
\end{aligned}
$$

If $\mathbf{y}\left(\mathbf{x}+q_{i} e_{i}\right)=\mathbf{y}+e_{i}$, as we assume $r$ satisfies Property 1 , the following inequalities hold:

$$
\begin{aligned}
v^{(i)}\left(\mathbf{x}+\mathbf{b}+b_{k} e_{k}\right)-v^{(i)}\left(\mathbf{x}+b_{k} e_{k}\right) & \geq r\left(\mathbf{y}\left(\mathbf{x}+\mathbf{b}+b_{k} e_{k}+q_{i} e_{i}\right)\right)-r\left(\mathbf{y}\left(\mathbf{x}+b_{k} e_{k}+q_{i} e_{i}\right)\right) \\
& =r\left(\mathbf{y}+\mathbf{e}+e_{k}+e_{i}\right)-r\left(\mathbf{y}+e_{k}+e_{i}\right) \\
& \geq r\left(\mathbf{y}+\mathbf{e}+e_{i}\right)-r\left(\mathbf{y}+e_{i}\right) \\
& =r\left(\mathbf{y}\left(\mathbf{x}+\mathbf{b}+q_{i} e_{i}\right)\right)-r\left(\mathbf{y}\left(\mathbf{x}+q_{i} e_{i}\right)\right) \\
& \geq v^{(i)}(\mathbf{x}+\mathbf{b})-v^{(i)}(\mathbf{x})
\end{aligned}
$$

Hence our inequality holds in each of the possible scenarios. Thus $v^{(i)}$ satisfies Property (EC.1). 
- Next we show $v^{(i)}$ satisfies Property (EC.2), i.e., $v^{(i)}\left(\mathbf{x}+b_{k} e_{k}\right)-v^{(i)}(\mathbf{x}) \geq v^{(i)}\left(\mathbf{x}+b_{k} e_{k}+b_{j} e_{j}\right)-$ $v^{(i)}\left(\mathbf{x}+b_{j} e_{j}\right), \forall k \neq j$. Pick arbitrary $k \in\{1,2, . ., m\}$. Again, there are four different scenarios we need to consider depending on the optimal actions at $v^{(i)}\left(\mathbf{x}+b_{k} e_{k}\right)$ and $v^{(i)}\left(\mathbf{x}+b_{j} e_{j}\right)$ :

(1) Suppose that $v^{(i)}\left(\mathbf{x}+b_{k} e_{k}\right)=r\left(\mathbf{y}\left(\mathbf{x}+b_{k} e_{k}\right)\right)$ and $v^{(i)}\left(\mathbf{x}+b_{j} e_{j}\right)=r\left(\mathbf{y}\left(\mathbf{x}+b_{j} e_{j}\right)\right)$. As we assume $r$ satisfies Property 2, the following inequalities hold:

$$
\begin{aligned}
v^{(i)}\left(\mathbf{x}+b_{k} e_{k}\right)-v^{(i)}(\mathbf{x}) & \geq r\left(\mathbf{y}\left(\mathbf{x}+b_{k} e_{k}\right)\right)-r(\mathbf{y}(\mathbf{x})) \\
& =r\left(\mathbf{y}+e_{k}\right)-r(\mathbf{y}) \\
& \geq r\left(\mathbf{y}+e_{k}+e_{j}\right)-r\left(\mathbf{y}+e_{j}\right) \\
& =r\left(\mathbf{y}\left(\mathbf{x}+b_{k} e_{k}+b_{j} e_{j}\right)\right)-r\left(\mathbf{y}\left(\mathbf{x}+b_{j} e_{j}\right)\right) \\
& \geq v^{(i)}\left(\mathbf{x}+b_{k} e_{k}+b_{j} e_{j}\right)-v^{(i)}\left(\mathbf{x}+b_{j} e_{j}\right)
\end{aligned}
$$

(2) Suppose that $v^{(i)}\left(\mathbf{x}+b_{k} e_{k}\right)=r\left(\mathbf{y}\left(\mathbf{x}+b_{k} e_{k}+q_{i} e_{i}\right)\right)$ and $v^{(i)}\left(\mathbf{x}+b_{j} e_{j}\right)=r\left(\mathbf{y}\left(\mathbf{x}+b_{j} e_{j}\right)\right)$. Also suppose that $i=j$. Recall that we should have either $\mathbf{y}\left(\mathbf{x}+q_{j} e_{j}\right)=\mathbf{y}$ or $\mathbf{y}\left(\mathbf{x}+q_{j} e_{j}\right)=$ $\mathbf{y}+e_{j}$. If $\mathbf{y}\left(\mathbf{x}+q_{j} e_{j}\right)=\mathbf{y}$, as we assume $r$ satisfies Property 2 , the following inequalities hold:

$$
\begin{aligned}
v^{(i)}\left(\mathbf{x}+b_{k} e_{k}\right)-v^{(i)}(\mathbf{x}) & \geq r\left(\mathbf{y}\left(\mathbf{x}+b_{k} e_{k}+q_{i} e_{i}\right)\right)-r\left(\mathbf{y}\left(\mathbf{x}+q_{i} e_{i}\right)\right) \\
& =r\left(\mathbf{y}\left(\mathbf{x}+b_{k} e_{k}+q_{j} e_{j}\right)\right)-r\left(\mathbf{y}\left(\mathbf{x}+q_{j} e_{j}\right)\right) \\
& =r\left(\mathbf{y}+e_{k}\right)-r(\mathbf{y}) \\
& \geq r\left(\mathbf{y}+e_{k}+e_{j}\right)-r\left(\mathbf{y}+e_{j}\right) \\
& =r\left(\mathbf{y}\left(\mathbf{x}+b_{k} e_{k}+b_{j} e_{j}\right)\right)-r\left(\mathbf{y}\left(\mathbf{x}+b_{j} e_{j}\right)\right) \\
& \geq v^{(i)}\left(\mathbf{x}+b_{k} e_{k}+b_{j} e_{j}\right)-v^{(i)}\left(\mathbf{x}+b_{j} e_{j}\right)
\end{aligned}
$$

If $\mathbf{y}\left(\mathbf{x}+q_{j} e_{j}\right)=\mathbf{y}+e_{j}$, note that $\mathbf{y}\left(\mathbf{x}+q_{j} e_{j}\right)=\mathbf{y}\left(\mathbf{x}+b_{j} e_{j}\right)$. Thus:

$$
\begin{aligned}
v^{(i)}\left(\mathbf{x}+b_{k} e_{k}\right)-v^{(i)}(\mathbf{x}) & \geq r\left(\mathbf{y}\left(\mathbf{x}+b_{k} e_{k}+q_{i} e_{i}\right)\right)-r\left(\mathbf{y}\left(\mathbf{x}+q_{i} e_{i}\right)\right) \\
& =r\left(\mathbf{y}\left(\mathbf{x}+b_{k} e_{k}+q_{j} e_{j}\right)\right)-r\left(\mathbf{y}\left(\mathbf{x}+q_{j} e_{j}\right)\right) \\
& =r\left(\mathbf{y}+e_{k}+e_{j}\right)-r\left(\mathbf{y}+e_{j}\right) \\
& =r\left(\mathbf{y}\left(\mathbf{x}+b_{k} e_{k}+b_{j} e_{j}\right)\right)-r\left(\mathbf{y}\left(\mathbf{x}+b_{j} e_{j}\right)\right) \\
& \geq v^{(i)}\left(\mathbf{x}+b_{k} e_{k}+b_{j} e_{j}\right)-v^{(i)}\left(\mathbf{x}+b_{j} e_{j}\right)
\end{aligned}
$$


Now suppose that $i \neq j$. Again we should have either $\mathbf{y}\left(\mathbf{x}+q_{i} e_{i}\right)=\mathbf{y}$ or $\mathbf{y}\left(\mathbf{x}+q_{i} e_{i}\right)=\mathbf{y}+e_{i}$.

If $\mathbf{y}\left(\mathbf{x}+q_{i} e_{i}\right)=\mathbf{y}$, as we assume $r$ satisfies Property 2, the following inequalities hold:

$$
\begin{aligned}
v^{(i)}\left(\mathbf{x}+b_{k} e_{k}\right)-v^{(i)}(\mathbf{x}) & \geq r\left(\mathbf{y}\left(\mathbf{x}+b_{k} e_{k}+q_{i} e_{i}\right)\right)-r(\mathbf{y}(\mathbf{x})) \\
& =r\left(\mathbf{y}+e_{k}\right)-r(\mathbf{y}) \\
& \geq r(\mathbf{y})-r\left(\mathbf{y}+e_{j}\right)+r\left(\mathbf{y}+e_{k}+e_{j}\right)-r(\mathbf{y}) \\
& =r\left(\mathbf{y}+e_{k}+e_{j}\right)-r\left(\mathbf{y}+e_{j}\right) \\
& =r\left(\mathbf{y}\left(\mathbf{x}+b_{k} e_{k}+b_{j} e_{j}+q_{i} e_{i}\right)\right)-r\left(\mathbf{y}\left(\mathbf{x}+b_{j} e_{j}\right)\right) \\
& \geq v^{(i)}\left(\mathbf{x}+b_{k} e_{k}+b_{j} e_{j}\right)-v^{(i)}\left(\mathbf{x}+b_{j} e_{j}\right)
\end{aligned}
$$

If $\mathbf{y}\left(\mathbf{x}+q_{i} e_{i}\right)=\mathbf{y}+e_{i}$, as we assume $r$ satisfies Property 2, the following inequalities hold:

$$
\begin{aligned}
v^{(i)}\left(\mathbf{x}+b_{k} e_{k}\right)-v^{(i)}(\mathbf{x}) & \geq r\left(\mathbf{y}\left(\mathbf{x}+b_{k} e_{k}+q_{i} e_{i}\right)\right)-r(\mathbf{y}(\mathbf{x})) \\
& =r\left(\mathbf{y}+e_{k}+e_{i}\right)-r(\mathbf{y}) \\
& \geq r\left(\mathbf{y}+e_{i}\right)-r\left(\mathbf{y}+e_{j}+e_{i}\right)+r\left(\mathbf{y}+e_{k}+e_{j}+e_{i}\right)-r(\mathbf{y}) \\
& \geq r\left(\mathbf{y}+e_{k}+e_{j}+e_{i}\right)-r\left(\mathbf{y}+e_{j}\right) \\
& =r\left(\mathbf{y}\left(\mathbf{x}+b_{k} e_{k}+b_{j} e_{j}+q_{i} e_{i}\right)\right)-r\left(\mathbf{y}\left(\mathbf{x}+b_{j} e_{j}\right)\right) \\
& \geq v^{(i)}\left(\mathbf{x}+b_{k} e_{k}+b_{j} e_{j}\right)-v^{(i)}\left(\mathbf{x}+b_{j} e_{j}\right)
\end{aligned}
$$

(3) Suppose that $v^{(i)}\left(\mathbf{x}+b_{k} e_{k}\right)=r\left(\mathbf{y}\left(\mathbf{x}+b_{k} e_{k}\right)\right)$ and $v^{(i)}\left(\mathbf{x}+b_{j} e_{j}\right)=r\left(\mathbf{y}\left(\mathbf{x}+b_{j} e_{j}+q_{i} e_{i}\right)\right)$. Also suppose that $i=k$. Again we should have either $\mathbf{y}\left(\mathbf{x}+q_{k} e_{k}\right)=\mathbf{y}$ or $\mathbf{y}\left(\mathbf{x}+q_{k} e_{k}\right)=\mathbf{y}+e_{k}$. If $\mathbf{y}\left(\mathbf{x}+q_{k} e_{k}\right)=\mathbf{y}$, as we assume $r$ satisfies Property 2, the following inequalities hold:

$$
\begin{aligned}
v^{(i)}\left(\mathbf{x}+b_{k} e_{k}\right)-v^{(i)}(\mathbf{x}) & \geq r\left(\mathbf{y}\left(\mathbf{x}+b_{k} e_{k}\right)\right)-r\left(\mathbf{y}\left(\mathbf{x}+q_{i} e_{i}\right)\right) \\
& =r\left(\mathbf{y}\left(\mathbf{x}+b_{k} e_{k}\right)\right)-r\left(\mathbf{y}\left(\mathbf{x}+q_{k} e_{k}\right)\right) \\
& =r\left(\mathbf{y}+e_{k}\right)-r(\mathbf{y}) \\
& \geq r\left(\mathbf{y}+e_{k}+e_{j}\right)-r\left(\mathbf{y}+e_{j}\right) \\
& =r\left(\mathbf{y}\left(\mathbf{x}+b_{k} e_{k}+b_{j} e_{j}\right)\right)-r\left(\mathbf{y}\left(\mathbf{x}+b_{j} e_{j}+q_{k} e_{k}\right)\right) \\
& =r\left(\mathbf{y}\left(\mathbf{x}+b_{k} e_{k}+b_{j} e_{j}\right)\right)-r\left(\mathbf{y}\left(\mathbf{x}+b_{j} e_{j}+q_{i} e_{i}\right)\right) \\
& \geq v^{(i)}\left(\mathbf{x}+b_{k} e_{k}+b_{j} e_{j}\right)-v^{(i)}\left(\mathbf{x}+b_{j} e_{j}\right)
\end{aligned}
$$

If $\mathbf{y}\left(\mathbf{x}+q_{k} e_{k}\right)=\mathbf{y}+e_{k}$, then it is easy to verify that

$$
v^{(i)}\left(\mathbf{x}+b_{k} e_{k}\right)-v^{(i)}(\mathbf{x}) \geq r\left(\mathbf{y}\left(\mathbf{x}+b_{k} e_{k}\right)\right)-r\left(\mathbf{y}\left(\mathbf{x}+q_{i} e_{i}\right)\right)
$$




$$
\begin{aligned}
& =r\left(\mathbf{y}\left(\mathbf{x}+b_{k} e_{k}\right)\right)-r\left(\mathbf{y}\left(\mathbf{x}+q_{k} e_{k}\right)\right) \\
& =r\left(\mathbf{y}+e_{k}\right)-r\left(\mathbf{y}+e_{k}\right) \\
& =r\left(\mathbf{y}+e_{k}+e_{j}\right)-r\left(\mathbf{y}+e_{j}+e_{k}\right) \\
& =r\left(\mathbf{y}\left(\mathbf{x}+b_{k} e_{k}+b_{j} e_{j}\right)\right)-r\left(\mathbf{y}\left(\mathbf{x}+b_{j} e_{j}+q_{k} e_{k}\right)\right) \\
& =r\left(\mathbf{y}\left(\mathbf{x}+b_{k} e_{k}+b_{j} e_{j}\right)\right)-r\left(\mathbf{y}\left(\mathbf{x}+b_{j} e_{j}+q_{i} e_{i}\right)\right) \\
& \geq v^{(i)}\left(\mathbf{x}+b_{k} e_{k}+b_{j} e_{j}\right)-v^{(i)}\left(\mathbf{x}+b_{j} e_{j}\right)
\end{aligned}
$$

Now suppose that $i \neq k$. Again we should have either $\mathbf{y}\left(\mathbf{x}+q_{i} e_{i}\right)=\mathbf{y}$ or $\mathbf{y}\left(\mathbf{x}+q_{i} e_{i}\right)=\mathbf{y}+e_{i}$. If $\mathbf{y}\left(\mathbf{x}+q_{i} e_{i}\right)=\mathbf{y}$, as we assume $r$ satisfies Property 2, the following inequalities hold:

$$
\begin{aligned}
v^{(i)}\left(\mathbf{x}+b_{k} e_{k}\right)-v^{(i)}(\mathbf{x}) & \geq r\left(\mathbf{y}\left(\mathbf{x}+b_{k} e_{k}\right)\right)-r(\mathbf{y}(\mathbf{x})) \\
& =r\left(\mathbf{y}+e_{k}\right)-r(\mathbf{y}) \\
& \geq r\left(\mathbf{y}+e_{k}+e_{j}\right)-r\left(\mathbf{y}+e_{j}\right) \\
& =r\left(\mathbf{y}\left(\mathbf{x}+b_{k} e_{k}+b_{j} e_{j}+q_{i} e_{i}\right)\right)-r\left(\mathbf{y}\left(\mathbf{x}+b_{j} e_{j}+q_{i} e_{i}\right)\right) \\
& \geq v^{(i)}\left(\mathbf{x}+b_{k} e_{k}+b_{j} e_{j}\right)-v^{(i)}\left(\mathbf{x}+b_{j} e_{j}\right)
\end{aligned}
$$

If $\mathbf{y}\left(\mathbf{x}+q_{i} e_{i}\right)=\mathbf{y}+e_{i}$, as we assume $r$ satisfies Property 2, the following inequalities hold:

$$
\begin{aligned}
v^{(i)}\left(\mathbf{x}+b_{k} e_{k}\right)-v^{(i)}(\mathbf{x}) & \geq r\left(\mathbf{y}\left(\mathbf{x}+b_{k} e_{k}\right)\right)-r(\mathbf{y}(\mathbf{x})) \\
& =r\left(\mathbf{y}+e_{k}\right)-r(\mathbf{y}) \\
& \geq r\left(\mathbf{y}+e_{k}+e_{j}\right)-r\left(\mathbf{y}+e_{j}\right) \\
& \geq r\left(\mathbf{y}+e_{k}+e_{j}+e_{i}\right)-r\left(\mathbf{y}+e_{j}+e_{i}\right) \\
& =r\left(\mathbf{y}\left(\mathbf{x}+b_{k} e_{k}+b_{j} e_{j}+q_{i} e_{i}\right)\right)-r\left(\mathbf{y}\left(\mathbf{x}+b_{j} e_{j}+q_{i} e_{i}\right)\right) \\
& \geq v^{(i)}\left(\mathbf{x}+b_{k} e_{k}+b_{j} e_{j}\right)-v^{(i)}\left(\mathbf{x}+b_{j} e_{j}\right)
\end{aligned}
$$

(4) Suppose that $v^{(i)}\left(\mathbf{x}+b_{k} e_{k}\right)=r\left(\mathbf{y}\left(\mathbf{x}+b_{k} e_{k}+q_{i} e_{i}\right)\right)$ and $v^{(i)}\left(\mathbf{x}+b_{j} e_{j}\right)=r\left(\mathbf{y}\left(\mathbf{x}+b_{j} e_{j}+q_{i} e_{i}\right)\right)$. Again we should have either $\mathbf{y}\left(\mathbf{x}+q_{i} e_{i}\right)=\mathbf{y}$ or $\mathbf{y}\left(\mathbf{x}+q_{i} e_{i}\right)=\mathbf{y}+e_{i}$. If $\mathbf{y}\left(\mathbf{x}+q_{i} e_{i}\right)=\mathbf{y}$, as we assume $r$ satisfies Property 2, the following inequalities hold:

$$
\begin{aligned}
v^{(i)}\left(\mathbf{x}+b_{k} e_{k}\right)-v^{(i)}(\mathbf{x}) & \geq r\left(\mathbf{y}\left(\mathbf{x}+b_{k} e_{k}+q_{i} e_{i}\right)\right)-r\left(\mathbf{y}\left(\mathbf{x}+q_{i} e_{i}\right)\right) \\
& =r\left(\mathbf{y}+e_{k}\right)-r(\mathbf{y}) \\
& \geq r\left(\mathbf{y}+e_{k}+e_{j}\right)-r\left(\mathbf{y}+e_{j}\right)
\end{aligned}
$$




$$
\begin{aligned}
& =r\left(\mathbf{y}\left(\mathbf{x}+b_{k} e_{k}+b_{j} e_{j}+q_{i} e_{i}\right)\right)-r\left(\mathbf{y}\left(\mathbf{x}+b_{j} e_{j}+q_{i} e_{i}\right)\right) \\
& \geq v^{(i)}\left(\mathbf{x}+b_{k} e_{k}+b_{j} e_{j}\right)-v^{(i)}\left(\mathbf{x}+b_{j} e_{j}\right)
\end{aligned}
$$

If $\mathbf{y}\left(\mathbf{x}+q_{i} e_{i}\right)=\mathbf{y}+e_{i}$, as we assume $r$ satisfies Property 2, the following inequalities hold:

$$
\begin{aligned}
v^{(i)}\left(\mathbf{x}+b_{k} e_{k}\right)-v^{(i)}(\mathbf{x}) & \geq r\left(\mathbf{y}\left(\mathbf{x}+b_{k} e_{k}+q_{i} e_{i}\right)\right)-r\left(\mathbf{y}\left(\mathbf{x}+q_{i} e_{i}\right)\right) \\
& =r\left(\mathbf{y}+e_{k}+e_{i}\right)-r\left(\mathbf{y}+e_{i}\right) \\
& \geq r\left(\mathbf{y}+e_{k}+e_{j}+e_{i}\right)-r\left(\mathbf{y}+e_{j}+e_{i}\right) \\
& =r\left(\mathbf{y}\left(\mathbf{x}+b_{k} e_{k}+b_{j} e_{j}+q_{i} e_{i}\right)\right)-r\left(\mathbf{y}\left(\mathbf{x}+b_{j} e_{j}+q_{i} e_{i}\right)\right) \\
& \geq v^{(i)}\left(\mathbf{x}+b_{k} e_{k}+b_{j} e_{j}\right)-v^{(i)}\left(\mathbf{x}+b_{j} e_{j}\right)
\end{aligned}
$$

Hence our inequality holds in each of the possible scenarios. Thus $v^{(i)}$ satisfies Property (EC.2).

(c) Assume $r$ satisfies Properties 1 and 2. We will show $v_{j}$ satisfies Properties (EC.1) and (EC.2).

- First we show $v_{j}$ satisfies Property (EC.1), i.e., $v_{j}\left(\mathbf{x}+\mathbf{b}+b_{i} e_{i}\right)-v_{j}\left(\mathbf{x}+b_{i} e_{i}\right) \geq v_{j}(\mathbf{x}+\mathbf{b})-v_{j}(\mathbf{x})$, $\forall i$. Recall that $d_{\mathbf{z}}$ can be positive only for original states $\mathbf{x}$ such that either $z_{i} \geq \max _{j}\left\{a_{i j}\right\}, \forall i$, or $z_{i}<\min _{j}\left\{a_{i j}\right\}, \forall i$. First we restrict ourselves to original states $\mathbf{x}$ such that $z_{i} \geq \max _{j}\left\{a_{i j}\right\}$, $\forall i$. Note that $\mathbf{y}\left(\mathbf{x}-\mathbf{a}_{j}\right)=\mathbf{y}(\mathbf{x})$ in this case. Thus $v_{j}(\mathbf{x})=r\left(\mathbf{y}\left(\mathbf{x}-\mathbf{a}_{j}\right)\right)=r(\mathbf{y}(\mathbf{x}))<r(\mathbf{y}(\mathbf{x}))+c_{j}$. Likewise $v_{j}\left(\mathbf{x}+b_{k} e_{k}\right)=r\left(\mathbf{y}\left(\mathbf{x}+b_{k} e_{k}-\mathbf{a}_{j}\right)\right), v_{j}(\mathbf{x}+\mathbf{b})=r\left(\mathbf{y}\left(\mathbf{x}+\mathbf{b}-\mathbf{a}_{j}\right)\right)$, and $v_{j}\left(\mathbf{x}+\mathbf{b}+b_{k} e_{k}\right)=$ $r\left(\mathbf{y}\left(\mathbf{x}+\mathbf{b}+b_{k} e_{k}-\mathbf{a}_{j}\right)\right)$. Since $r$ satisfies Property 1 , the following inequality holds:

$$
\begin{aligned}
v_{j}\left(\mathbf{x}+\mathbf{b}+b_{k} e_{k}\right)-v_{j}\left(\mathbf{x}+b_{k} e_{k}\right) & =r\left(\mathbf{y}\left(\mathbf{x}+\mathbf{b}+b_{k} e_{k}-\mathbf{a}_{j}\right)\right)-r\left(\mathbf{y}\left(\mathbf{x}+b_{k} e_{k}-\mathbf{a}_{j}\right)\right) \\
& =r\left(\mathbf{y}+\mathbf{e}+e_{k}\right)-r\left(\mathbf{y}+e_{k}\right) \\
& \geq r(\mathbf{y}+\mathbf{e})-r(\mathbf{y}) \\
& =r\left(\mathbf{y}\left(\mathbf{x}+\mathbf{b}-\mathbf{a}_{j}\right)\right)-r\left(\mathbf{y}\left(\mathbf{x}-\mathbf{a}_{j}\right)\right) \\
& =v_{j}(\mathbf{x}+\mathbf{b})-v_{j}(\mathbf{x})
\end{aligned}
$$

Now we restrict ourselves to original states $\mathbf{x}$ such that $z_{i}<\min _{j}\left\{a_{i j}\right\}, \forall i$. We need to consider four different scenarios depending on the optimal actions at $v_{j}\left(\mathbf{x}+\mathbf{b}+b_{k} e_{k}\right)$ and $v_{j}(\mathbf{x})$ :

(1) Suppose that $v_{j}\left(\mathbf{x}+\mathbf{b}+b_{k} e_{k}\right)=r\left(\mathbf{y}\left(\mathbf{x}+\mathbf{b}+b_{k} e_{k}\right)\right)+c_{j}$ and $v_{j}(\mathbf{x})=r(\mathbf{y}(\mathbf{x}))+c_{j}$. As we assume $r$ satisfies Property 1, the following inequalities hold:

$$
\begin{aligned}
v_{j}\left(\mathbf{x}+\mathbf{b}+b_{k} e_{k}\right)-v_{j}\left(\mathbf{x}+b_{k} e_{k}\right) & \geq r\left(\mathbf{y}\left(\mathbf{x}+\mathbf{b}+b_{k} e_{k}\right)\right)+c_{j}-r\left(\mathbf{y}\left(\mathbf{x}+b_{k} e_{k}\right)\right)-c_{j} \\
& =r\left(\mathbf{y}+\mathbf{e}+e_{k}\right)-r\left(\mathbf{y}+e_{k}\right)
\end{aligned}
$$




$$
\begin{aligned}
& \geq r(\mathbf{y}+\mathbf{e})-r(\mathbf{y}) \\
& =r(\mathbf{y}(\mathbf{x}+\mathbf{b}))+c_{j}-r(\mathbf{y}(\mathbf{x}))-c_{j} \\
& \geq v_{j}(\mathbf{x}+\mathbf{b})-v_{j}(\mathbf{x})
\end{aligned}
$$

(2) Suppose that $v_{j}\left(\mathbf{x}+\mathbf{b}+b_{k} e_{k}\right)=r\left(\mathbf{y}\left(\mathbf{x}+\mathbf{b}+b_{k} e_{k}-\mathbf{a}_{j}\right)\right)$ and $v_{j}(\mathbf{x})=r(\mathbf{y}(\mathbf{x}))+c_{j}$. As we consider original states $\mathbf{x}$ such that $z_{i}<\min _{j}\left\{a_{i j}\right\}, \forall i$, we should have $\mathbf{y}\left(\mathbf{x}+\mathbf{b}-\mathbf{a}_{j}\right)=\mathbf{y}(\mathbf{x})$. Thus:

$$
\begin{aligned}
v_{j}\left(\mathbf{x}+\mathbf{b}+b_{k} e_{k}\right)-v_{j}\left(\mathbf{x}+b_{k} e_{k}\right) & \geq r\left(\mathbf{y}\left(\mathbf{x}+\mathbf{b}+b_{k} e_{k}-\mathbf{a}_{j}\right)\right)-r\left(\mathbf{y}\left(\mathbf{x}+b_{k} e_{k}\right)\right)-c_{j} \\
& =r\left(\mathbf{y}+e_{k}\right)-r\left(\mathbf{y}+e_{k}\right)-c_{j} \\
& =r(\mathbf{y})-r(\mathbf{y})-c_{j} \\
& =r\left(\mathbf{y}\left(\mathbf{x}+\mathbf{b}-\mathbf{a}_{j}\right)\right)-r(\mathbf{y}(\mathbf{x}))-c_{j} \\
& \geq v_{j}(\mathbf{x}+\mathbf{b})-v_{j}(\mathbf{x})
\end{aligned}
$$

(3) Suppose that $v_{j}\left(\mathbf{x}+\mathbf{b}+b_{k} e_{k}\right)=r\left(\mathbf{y}\left(\mathbf{x}+\mathbf{b}+b_{k} e_{k}\right)\right)+c_{j}$ and $v_{j}(\mathbf{x})=r\left(\mathbf{y}\left(\mathbf{x}-\mathbf{a}_{j}\right)\right)$. Note that we should have $\mathbf{x} \geq \mathbf{a}_{j}$ for this case to exist. As we consider original states $\mathbf{x}$ such that $z_{i}<\min _{j}\left\{a_{i j}\right\}, \forall i$, we should also have $\mathbf{y}\left(\mathbf{x}-\mathbf{a}_{j}\right)=\mathbf{y}(\mathbf{x})-\mathbf{e}$. As we assume $r$ satisfies Property 1, the following inequalities hold:

$$
\begin{aligned}
v_{j}\left(\mathbf{x}+\mathbf{b}+b_{k} e_{k}\right)-v_{j}\left(\mathbf{x}+b_{k} e_{k}\right) & \geq r\left(\mathbf{y}\left(\mathbf{x}+\mathbf{b}+b_{k} e_{k}\right)\right)+c_{j}-r\left(\mathbf{y}\left(\mathbf{x}+b_{k} e_{k}-\mathbf{a}_{j}\right)\right) \\
& =r\left(\mathbf{y}+\mathbf{e}+e_{k}\right)+c_{j}-r\left(\mathbf{y}+e_{k}-\mathbf{e}\right) \\
& \geq r(\mathbf{y}+\mathbf{e})+c_{j}-r(\mathbf{y})+r\left(\mathbf{y}+e_{k}\right)-r\left(\mathbf{y}+e_{k}-\mathbf{e}\right) \\
& \geq r(\mathbf{y}+\mathbf{e})+c_{j}-r(\mathbf{y}-\mathbf{e}) \\
& =r(\mathbf{y}(\mathbf{x}+\mathbf{b}))+c_{j}-r\left(\mathbf{y}\left(\mathbf{x}-\mathbf{a}_{j}\right)\right) \\
& \geq v_{j}(\mathbf{x}+\mathbf{b})-v_{j}(\mathbf{x})
\end{aligned}
$$

(4) Suppose that $v_{j}\left(\mathbf{x}+\mathbf{b}+b_{k} e_{k}\right)=r\left(\mathbf{y}\left(\mathbf{x}+\mathbf{b}+b_{k} e_{k}-\mathbf{a}_{j}\right)\right)$ and $v_{j}(\mathbf{x})=r\left(\mathbf{y}\left(\mathbf{x}-\mathbf{a}_{j}\right)\right)$. Again we should have $\mathbf{x} \geq \mathbf{a}_{j}$ for this case to exist. Also recall that $\mathbf{y}\left(\mathbf{x}-\mathbf{a}_{j}\right)=\mathbf{y}(\mathbf{x})-\mathbf{e}$. As we assume $r$ satisfies Property 1, the following inequalities hold:

$$
\begin{aligned}
v_{j}\left(\mathbf{x}+\mathbf{b}+b_{k} e_{k}\right)-v_{j}\left(\mathbf{x}+b_{k} e_{k}\right) & \geq r\left(\mathbf{y}\left(\mathbf{x}+\mathbf{b}+b_{k} e_{k}-\mathbf{a}_{j}\right)\right)-r\left(\mathbf{y}\left(\mathbf{x}+b_{k} e_{k}-\mathbf{a}_{j}\right)\right) \\
& =r\left(\mathbf{y}+e_{k}\right)-r\left(\mathbf{y}+e_{k}-\mathbf{e}\right) \\
& \geq r(\mathbf{y})-r(\mathbf{y}-\mathbf{e})
\end{aligned}
$$




$$
\begin{aligned}
& =r\left(\mathbf{y}\left(\mathbf{x}+\mathbf{b}-\mathbf{a}_{j}\right)\right)-r\left(\mathbf{y}\left(\mathbf{x}-\mathbf{a}_{j}\right)\right) \\
& \geq v_{j}(\mathbf{x}+\mathbf{b})-v_{j}(\mathbf{x})
\end{aligned}
$$

Hence our inequality holds in each of the possible scenarios. Thus $v_{j}$ satisfies Property (EC.1).

- Next we show $v_{j}$ satisfies Property (EC.2), i.e., $v_{j}\left(\mathbf{x}+b_{i} e_{i}\right)-v_{j}(\mathbf{x}) \geq v_{j}\left(\mathbf{x}+b_{i} e_{i}+b_{k} e_{k}\right)-$ $v_{j}\left(\mathbf{x}+b_{k} e_{k}\right), \forall i \neq k$. Pick arbitrary $i \in\{1,2, . ., m\}$. First we restrict ourselves to original states $\mathbf{x}$ such that $z_{i} \geq \max _{j}\left\{a_{i j}\right\}, \forall i$. Note that $\mathbf{y}\left(\mathbf{x}-\mathbf{a}_{j}\right)=\mathbf{y}(\mathbf{x})$ in this case. Thus $v_{j}(\mathbf{x})=$ $r\left(\mathbf{y}\left(\mathbf{x}-\mathbf{a}_{j}\right)\right)=r(\mathbf{y}(\mathbf{x}))<r(\mathbf{y}(\mathbf{x}))+c_{j}$. Likewise $v_{j}\left(\mathbf{x}+b_{i} e_{i}\right)=r\left(\mathbf{y}\left(\mathbf{x}+b_{i} e_{i}-\mathbf{a}_{j}\right)\right), v_{j}\left(\mathbf{x}+b_{k} e_{k}\right)=$ $r\left(\mathbf{y}\left(\mathbf{x}+b_{k} e_{k}-\mathbf{a}_{j}\right)\right)$, and $v_{j}\left(\mathbf{x}+b_{i} e_{i}+b_{k} e_{k}\right)=r\left(\mathbf{y}\left(\mathbf{x}+b_{i} e_{i}+b_{k} e_{k}-\mathbf{a}_{j}\right)\right)$. Since $r$ satisfies Property 2, the following inequality holds:

$$
\begin{aligned}
v_{j}\left(\mathbf{x}+b_{i} e_{i}\right)-v_{j}(\mathbf{x}) & =r\left(\mathbf{y}\left(\mathbf{x}+b_{i} e_{i}-\mathbf{a}_{j}\right)\right)-r\left(\mathbf{y}\left(\mathbf{x}-\mathbf{a}_{j}\right)\right) \\
& =r\left(\mathbf{y}+e_{i}\right)-r(\mathbf{y}) \\
& \geq r\left(\mathbf{y}+e_{i}+e_{k}\right)-r\left(\mathbf{y}+e_{k}\right) \\
& =r\left(\mathbf{y}\left(\mathbf{x}+b_{i} e_{i}+b_{k} e_{k}-\mathbf{a}_{j}\right)\right)-r\left(\mathbf{y}\left(\mathbf{x}+b_{k} e_{k}-\mathbf{a}_{j}\right)\right) \\
& =v_{j}\left(\mathbf{x}+b_{i} e_{i}+b_{k} e_{k}\right)-v_{j}\left(\mathbf{x}+b_{k} e_{k}\right)
\end{aligned}
$$

Now we restrict ourselves to original states $\mathbf{x}$ such that $z_{i}<\min _{j}\left\{a_{i j}\right\}, \forall i$. We need to consider four different scenarios depending on the optimal actions at $v_{j}\left(\mathbf{x}+b_{i} e_{i}\right)$ and $v_{j}\left(\mathbf{x}+b_{k} e_{k}\right)$ :

(1) Suppose that $v_{j}\left(\mathbf{x}+b_{i} e_{i}\right)=r\left(\mathbf{y}\left(\mathbf{x}+b_{i} e_{i}\right)\right)+c_{j}$ and $v_{j}\left(\mathbf{x}+b_{k} e_{k}\right)=r\left(\mathbf{y}\left(\mathbf{x}+b_{k} e_{k}\right)\right)+c_{j}$. As we assume $r$ satisfies Property 2, the following inequalities hold:

$$
\begin{aligned}
v_{j}\left(\mathbf{x}+b_{i} e_{i}\right)-v_{j}(\mathbf{x}) & \geq r\left(\mathbf{y}\left(\mathbf{x}+b_{i} e_{i}\right)\right)+c_{j}-r(\mathbf{y}(\mathbf{x}))-c_{j} \\
& =r\left(\mathbf{y}+e_{i}\right)-r(\mathbf{y}) \\
& \geq r\left(\mathbf{y}+e_{i}+e_{k}\right)-r\left(\mathbf{y}+e_{k}\right) \\
& =r\left(\mathbf{y}\left(\mathbf{x}+b_{i} e_{i}+b_{k} e_{k}\right)\right)+c_{j}-r\left(\mathbf{y}\left(\mathbf{x}+b_{k} e_{k}\right)\right)-c_{j} \\
& \geq v_{j}\left(\mathbf{x}+b_{i} e_{i}+b_{k} e_{k}\right)-v_{j}\left(\mathbf{x}+b_{k} e_{k}\right)
\end{aligned}
$$

(2) Suppose that $v_{j}\left(\mathbf{x}+b_{i} e_{i}\right)=r\left(\mathbf{y}\left(\mathbf{x}+b_{i} e_{i}-\mathbf{a}_{j}\right)\right)$ and $v_{j}\left(\mathbf{x}+b_{k} e_{k}\right)=r\left(\mathbf{y}\left(\mathbf{x}+b_{k} e_{k}\right)\right)+c_{j}$. Note that we should have $\mathbf{x}+b_{i} e_{i} \geq \mathbf{a}_{j}$ for this case to exist. As we consider original states $\mathbf{x}$ such that $z_{i}<\min _{j}\left\{a_{i j}\right\}, \forall i$, we should also have $\mathbf{y}\left(\mathbf{x}+b_{i} e_{i}-\mathbf{a}_{j}\right)=\mathbf{y}(\mathbf{x})+e_{i}-\mathbf{e}$ and $\mathbf{y}\left(\mathbf{x}+b_{i} e_{i}+b_{k} e_{k}-\mathbf{a}_{j}\right)=\mathbf{y}(\mathbf{x})+e_{i}+e_{k}-\mathbf{e}$. As we assume $r$ satisfies Properties 1 and 2, the following inequalities hold:

$$
v_{j}\left(\mathbf{x}+b_{i} e_{i}\right)-v_{j}(\mathbf{x}) \geq r\left(\mathbf{y}\left(\mathbf{x}+b_{i} e_{i}-\mathbf{a}_{j}\right)\right)-r(\mathbf{y}(\mathbf{x}))-c_{j}
$$




$$
\begin{aligned}
& =r\left(\mathbf{y}+e_{i}-\mathbf{e}\right)-r(\mathbf{y})-c_{j} \\
& \geq r\left(\mathbf{y}+e_{i}\right)-r\left(\mathbf{y}+e_{i}+e_{k}\right)+r\left(\mathbf{y}+e_{i}+e_{k}-\mathbf{e}\right)-r(\mathbf{y})-c_{j} \\
& \geq r\left(\mathbf{y}+e_{i}+e_{k}-\mathbf{e}\right)-r\left(\mathbf{y}+e_{k}\right)-c_{j} \\
& =r\left(\mathbf{y}\left(\mathbf{x}+b_{i} e_{i}+b_{k} e_{k}-\mathbf{a}_{j}\right)\right)-r\left(\mathbf{y}\left(\mathbf{x}+b_{k} e_{k}\right)\right)-c_{j} \\
& \geq v_{j}\left(\mathbf{x}+b_{i} e_{i}+b_{k} e_{k}\right)-v_{j}\left(\mathbf{x}+b_{k} e_{k}\right)
\end{aligned}
$$

(3) Suppose that $v_{j}\left(\mathbf{x}+b_{i} e_{i}\right)=r\left(\mathbf{y}\left(\mathbf{x}+b_{i} e_{i}\right)\right)+c_{j}$ and $v_{j}\left(\mathbf{x}+b_{k} e_{k}\right)=r\left(\mathbf{y}\left(\mathbf{x}+b_{k} e_{k}-\mathbf{a}_{j}\right)\right)$. Note that we should have $\mathbf{x}+b_{k} e_{k} \geq \mathbf{a}_{j}$ for this case to exist. Again, as we consider original states $\mathbf{x}$ such that $z_{i}<\min _{j}\left\{a_{i j}\right\}, \forall i$, we should also have $\mathbf{y}\left(\mathbf{x}+b_{k} e_{k}-\mathbf{a}_{j}\right)=\mathbf{y}(\mathbf{x})+e_{k}-\mathbf{e}$ and $\mathbf{y}\left(\mathbf{x}+b_{i} e_{i}+b_{k} e_{k}-\mathbf{a}_{j}\right)=\mathbf{y}(\mathbf{x})+e_{i}+e_{k}-\mathbf{e}$. As we assume $r$ satisfies Properties 1 and 2, the following inequalities hold:

$$
\begin{aligned}
v_{j}\left(\mathbf{x}+b_{i} e_{i}\right)-v_{j}(\mathbf{x}) & \geq r\left(\mathbf{y}\left(\mathbf{x}+b_{i} e_{i}\right)\right)+c_{j}-r(\mathbf{y}(\mathbf{x}))-c_{j} \\
& =r\left(\mathbf{y}+e_{i}\right)-r(\mathbf{y}) \\
& \geq r\left(\mathbf{y}+e_{i}+e_{k}\right)-r\left(\mathbf{y}+e_{k}\right) \\
& \geq r\left(\mathbf{y}+e_{i}+e_{k}-\mathbf{e}\right)-r\left(\mathbf{y}+e_{k}-\mathbf{e}\right) \\
& =r\left(\mathbf{y}\left(\mathbf{x}+b_{i} e_{i}+b_{k} e_{k}-\mathbf{a}_{j}\right)\right)-r\left(\mathbf{y}\left(\mathbf{x}+b_{k} e_{k}-\mathbf{a}_{j}\right)\right) \\
& \geq v_{j}\left(\mathbf{x}+b_{i} e_{i}+b_{k} e_{k}\right)-v_{j}\left(\mathbf{x}+b_{k} e_{k}\right)
\end{aligned}
$$

(4) Suppose that $v_{j}\left(\mathbf{x}+b_{i} e_{i}\right)=r\left(\mathbf{y}\left(\mathbf{x}+b_{i} e_{i}-\mathbf{a}_{j}\right)\right)$ and $v_{j}\left(\mathbf{x}+b_{k} e_{k}\right)=r\left(\mathbf{y}\left(\mathbf{x}+b_{k} e_{k}-\mathbf{a}_{j}\right)\right)$. Note that we should have $\mathbf{x}+b_{i} e_{i} \geq \mathbf{a}_{j}$ and $\mathbf{x}+b_{k} e_{k} \geq \mathbf{a}_{j}$, implying $\mathbf{x} \geq \mathbf{a}_{j}$, for this case to exist. Again, as we consider original states $\mathbf{x}$ such that $z_{i}<\min _{j}\left\{a_{i j}\right\}, \forall i$, we should also have $\mathbf{y}\left(\mathbf{x}-\mathbf{a}_{j}\right)=\mathbf{y}(\mathbf{x})-\mathbf{e}$. As we assume $r$ satisfies Property 2, the following inequalities hold:

$$
\begin{aligned}
v_{j}\left(\mathbf{x}+b_{i} e_{i}\right)-v_{j}(\mathbf{x}) & \geq r\left(\mathbf{y}\left(\mathbf{x}+b_{i} e_{i}-\mathbf{a}_{j}\right)\right)-r\left(\mathbf{y}\left(\mathbf{x}-\mathbf{a}_{j}\right)\right) \\
& =r\left(\mathbf{y}+e_{i}-\mathbf{e}\right)-r(\mathbf{y}-\mathbf{e}) \\
& \geq r\left(\mathbf{y}+e_{i}+e_{k}-\mathbf{e}\right)-r\left(\mathbf{y}+e_{k}-\mathbf{e}\right) \\
& =r\left(\mathbf{y}\left(\mathbf{x}+b_{i} e_{i}+b_{k} e_{k}-\mathbf{a}_{j}\right)\right)-r\left(\mathbf{y}\left(\mathbf{x}+b_{k} e_{k}-\mathbf{a}_{j}\right)\right) \\
& \geq v_{j}\left(\mathbf{x}+b_{i} e_{i}+b_{k} e_{k}\right)-v_{j}\left(\mathbf{x}+b_{k} e_{k}\right)
\end{aligned}
$$

Hence our inequality holds in each of the possible scenarios. Thus $v_{j}$ satisfies Property (EC.2). 
We showed that the function $h$ satisfies Properties (EC.1) and (EC.2), and if $r$ satisfies Properties 1 and 2, the functions $v^{(i)}$ and $v_{j}$ satisfy Properties (EC.1) and (EC.2), $\forall i, j$. Since Properties (EC.1) and (EC.2) are preserved by linear transformations, if $r$ satisfies Properties 1 and $2, v$ satisfies Properties (EC.1) and (EC.2), and thus Fr satisfies Properties 1 and 2.

Recall that $\mathcal{V}$ is the set of real-valued functions $f$ on $\mathbb{N}_{0}^{m}$ that satisfy Properties $1-3$. We will show that Properties 1 and 2 imply Property 3: Property 2 implies the following inequalities.

$$
\begin{aligned}
& f\left(\mathbf{y}+2 e_{i}\right)-f\left(\mathbf{y}+e_{i}\right) \geq f\left(\mathbf{y}+2 e_{i}+e_{1}\right)-f\left(\mathbf{y}+e_{i}+e_{1}\right), \\
& f\left(\mathbf{y}+2 e_{i}+e_{1}\right)-f\left(\mathbf{y}+e_{i}+e_{1}\right) \geq f\left(\mathbf{y}+2 e_{i}+e_{1}+e_{2}\right)-f\left(\mathbf{y}+e_{i}+e_{1}+e_{2}\right), \\
& \vdots \\
& f\left(\mathbf{y}+2 e_{i}+e_{1}+\cdots+e_{i-2}\right)-f\left(\mathbf{y}+e_{i}+e_{1}+\cdots+e_{i-2}\right) \\
& \geq f\left(\mathbf{y}+2 e_{i}+e_{1}+\cdots+e_{i-1}\right)-f\left(\mathbf{y}+e_{i}+e_{1}+\cdots+e_{i-1}\right) .
\end{aligned}
$$

Note that $f\left(\mathbf{y}+2 e_{i}+e_{1}+\cdots+e_{i-1}\right)-f\left(\mathbf{y}+e_{i}+e_{1}+\cdots+e_{i-1}\right)$ can be rewritten as $f\left(\mathbf{y}+e_{i}+e_{1}+\right.$ $\left.\cdots+e_{i}\right)-f\left(\mathbf{y}+e_{1}+\cdots+e_{i}\right)$. Property 2 also implies the following inequalities.

$$
\begin{aligned}
& f\left(\mathbf{y}+e_{i}+e_{1}+\cdots+e_{i}\right)-f\left(\mathbf{y}+e_{1}+\cdots+e_{i}\right) \geq f\left(\mathbf{y}+e_{i}+e_{1}+\cdots+e_{i+1}\right)-f\left(\mathbf{y}+e_{1}+\cdots+e_{i+1}\right) \\
& f\left(\mathbf{y}+e_{i}+e_{1}+\cdots+e_{i+1}\right)-f\left(\mathbf{y}+e_{1}+\cdots+e_{i+1}\right) \geq f\left(\mathbf{y}+e_{i}+e_{1}+\cdots+e_{i+2}\right)-f\left(\mathbf{y}+e_{1}+\cdots+e_{i+2}\right) \\
& \vdots \\
& f\left(\mathbf{y}+e_{i}+e_{1}+\cdots+e_{m-1}\right)-f\left(\mathbf{y}+e_{1}+\cdots+e_{m-1}\right) \geq f\left(\mathbf{y}+e_{i}+e_{1}+\cdots+e_{m}\right)-f\left(\mathbf{y}+e_{1}+\cdots+e_{m}\right)
\end{aligned}
$$

Summation of all of the above inequalities obtained from Property 2 implies that $f\left(\mathbf{y}+2 e_{i}\right)-$ $f\left(\mathbf{y}+e_{i}\right) \geq f\left(\mathbf{y}+e_{i}+\mathbf{e}\right)-f(\mathbf{y}+\mathbf{e})$. Notice that Property 1 implies that $f\left(\mathbf{y}+e_{i}+\mathbf{e}\right)-f(\mathbf{y}+\mathbf{e}) \geq$ $f\left(\mathbf{y}+e_{i}\right)-f(\mathbf{y})$. Summation of these two inequalities implies Property 3.

If $r$ satisfies Properties 1 and 2, since Properties 1 and 2 imply Property 3, $r$ satisfies Property 3 and $F r$ satisfies Properties 1-3. Hence $F: \mathcal{V} \rightarrow \mathcal{V}$. Following Propositions 3.1.5 and 3.1.6 in Bertsekas (2007), we verify that $\lim _{k \rightarrow \infty}\left(F^{k} r_{0}\right)(\mathbf{y})=r^{*}(\mathbf{y})$ where $r_{0}$ is the zero function, $r^{*}$ is the aggregate-optimal cost function, and $F^{k}$ refers to $k$ compositions of operator $F$. Since $r_{0} \in \mathcal{V}$ and $F: \mathcal{V} \rightarrow \mathcal{V}$, we have $F^{k} r_{0} \in \mathcal{V}$, and therefore $r^{*} \in \mathcal{V}$

Proof of Theorem 1. By Lemma 1, we know $r^{*} \in \mathcal{V}$. Define, for $r^{*} \in \mathcal{V}$,

$$
\begin{aligned}
& S_{i}^{*}(\mathbf{p})=\min \left\{\mathbf{p}+k \mathbf{b}: r^{*}\left(\mathbf{y}\left(\mathbf{p}+k \mathbf{b}+q_{i} e_{i}\right)\right)-r^{*}(\mathbf{y}(\mathbf{p}+k \mathbf{b}))>0, k \in \mathbb{N}_{0}\right\}, \forall i, \text { and } \\
& R_{j}^{*}(\mathbf{p})=\min \left\{\mathbf{p}+k \mathbf{b}: r^{*}(\mathbf{y}(\mathbf{p}+k \mathbf{b}))-r^{*}\left(\mathbf{y}\left(\mathbf{p}+k \mathbf{b}-\mathbf{a}_{j}\right)\right)>-c_{j}, k \in \mathbb{N}_{0}, \text { and } \mathbf{p}+k \mathbf{b} \geq \mathbf{a}_{j}\right\}, \forall j .
\end{aligned}
$$


(1) First suppose that $\mathbf{y}\left(\mathbf{p}+k \mathbf{b}+q_{i} e_{i}\right)=\mathbf{y}(\mathbf{p}+k \mathbf{b})+e_{i}$. Since $r^{*}$ satisfies Property $1, r^{*}(\mathbf{y}(\mathbf{p}+$ $\left.\left.k \mathbf{b}+q_{i} e_{i}\right)\right)-r^{*}(\mathbf{y}(\mathbf{p}+k \mathbf{b}))=r^{*}\left(\mathbf{y}(\mathbf{p}+k \mathbf{b})+e_{i}\right)-r^{*}(\mathbf{y}(\mathbf{p}+k \mathbf{b}))$ is nondecreasing in $k$. As $k$ increases, since the holding cost rate $h$ is strictly increasing, this difference will eventually cross 0 . Therefore, the lattice-dependent base-stock policy is optimal. Now suppose that $\mathbf{y}(\mathbf{p}+$ $\left.k \mathbf{b}+q_{i} e_{i}\right)=\mathbf{y}(\mathbf{p}+k \mathbf{b})$. Since we have $r^{*}\left(\mathbf{y}\left(\mathbf{p}+k \mathbf{b}+q_{i} e_{i}\right)\right)=r^{*}(\mathbf{y}(\mathbf{p}+k \mathbf{b}))$ in this case, the controller is always indifferent between producing and not producing a batch of component $i$ at aggregate-optimality.

(2) First suppose that $\mathbf{y}(\mathbf{p}+k \mathbf{b})=\mathbf{y}\left(\mathbf{p}+k \mathbf{b}-\mathbf{a}_{j}\right)+\sum_{i \in I} e_{i}$ where $I$ is an arbitrary nonempty subset of the set $\{1, . ., m\}$. Since $r^{*}$ satisfies Property $1, r\left(\mathbf{y}+\mathbf{e}+\sum_{i \in I} e_{i}\right)-r\left(\mathbf{y}+\sum_{i \in I} e_{i}\right) \geq$ $r(\mathbf{y}+\mathbf{e})-r(\mathbf{y})$. Thus $r^{*}(\mathbf{y}(\mathbf{p}+k \mathbf{b}))-r^{*}\left(\mathbf{y}\left(\mathbf{p}+k \mathbf{b}-\mathbf{a}_{j}\right)\right)=r^{*}\left(\mathbf{y}\left(\mathbf{p}+k \mathbf{b}-\mathbf{a}_{j}\right)+\sum_{i \in I} e_{i}\right)-$ $r^{*}\left(\mathbf{y}\left(\mathbf{p}+k \mathbf{b}-\mathbf{a}_{j}\right)\right)$ is nondecreasing in $k$. As $k$ increases, since the holding cost rate $h$ is strictly increasing, this difference will eventually cross $-c_{j}$. Therefore, the lattice-dependent rationing policy is optimal. Now suppose that $\mathbf{y}(\mathbf{p}+k \mathbf{b})=\mathbf{y}\left(\mathbf{p}+k \mathbf{b}-\mathbf{a}_{j}\right)$. Since we have $r^{*}(\mathbf{y}(\mathbf{p}+k \mathbf{b}))+c_{j}=r^{*}\left(\mathbf{y}\left(\mathbf{p}+k \mathbf{b}-\mathbf{a}_{j}\right)\right)+c_{j} \geq r^{*}\left(\mathbf{y}\left(\mathbf{p}+k \mathbf{b}-\mathbf{a}_{j}\right)\right)$ in this case, it is always aggregate-optimal to satisfy a demand for product $j$ on each of the lattices $\mathbb{L}(\mathbf{p}, \mathbf{b})$ with $\mathbf{y}\left(\mathbf{p}-\mathbf{a}_{j}\right)=\mathbf{y}(\mathbf{p})$. In other words, $R_{j}^{*}(\mathbf{p})=\mathbf{p}$ if $\left\lfloor\frac{p_{i}}{b_{i}}\right\rfloor=\left\lfloor\frac{p_{i}-a_{i j}}{b_{i}}\right\rfloor$ for all $i, \forall j$, and $\forall \mathbf{p}$.

Next we will prove properties (i)-(vii):

(i) Since $\mathbf{y}\left(\mathbf{p}+q_{i} e_{i}\right)=\mathbf{y}(\mathbf{p})$, we have $r^{*}\left(\mathbf{y}\left(\mathbf{p}+k \mathbf{b}+q_{i} e_{i}\right)\right)=r^{*}\left(\mathbf{y}\left(\mathbf{p}+q_{i} e_{i}\right)+k \mathbf{e}\right)=r^{*}(\mathbf{y}(\mathbf{p})+k \mathbf{e})=$ $r^{*}(\mathbf{y}(\mathbf{p}+k \mathbf{b})), \forall k \in \mathbb{N}_{0}$ : The controller is indifferent between producing and not producing a batch of component $i$ at aggregate-optimality.

(ii) Pick arbitrary $\mathbf{p}$ and $\mathbf{r}$ such that $\mathbf{y}(\mathbf{p})+e_{i}=\mathbf{y}(\mathbf{r})+e_{i}=\mathbf{y}\left(\mathbf{p}+q_{i} e_{i}\right)=\mathbf{y}\left(\mathbf{r}+q_{i} e_{i}\right)$. Suppose that it is aggregate-optimal to produce a batch of component $i$ in original state $\mathbf{x}_{1}=\mathbf{p}+k \mathbf{b}$ with $\mathbf{y}\left(\mathbf{x}_{1}\right)=\mathbf{y}_{1}$, i.e., $r^{*}\left(\mathbf{y}\left(\mathbf{x}_{1}+q_{i} e_{i}\right)\right)=r^{*}\left(\mathbf{y}_{1}+e_{i}\right) \leq r^{*}\left(\mathbf{y}_{1}\right)=r^{*}\left(\mathbf{y}\left(\mathbf{x}_{1}\right)\right)$. Notice that it is aggregateoptimal to produce a batch of component $i$ in original state $\mathbf{x}_{2}=\mathbf{r}+k \mathbf{b}$ since $\mathbf{y}\left(\mathbf{x}_{2}\right)=\mathbf{y}_{1}$ and $r^{*}\left(\mathbf{y}\left(\mathbf{x}_{2}+q_{i} e_{i}\right)\right)=r^{*}\left(\mathbf{y}_{1}+e_{i}\right) \leq r^{*}\left(\mathbf{y}_{1}\right)=r^{*}\left(\mathbf{y}\left(\mathbf{x}_{2}\right)\right)$. Likewise, it can be shown that if it is not aggregate-optimal to produce a batch of component $i$ in original state $\mathbf{x}_{1}$, it is not aggregate-optimal to produce a batch of component $i$ in original state $\mathbf{x}_{2}$. Hence we must have $\mathbf{y}\left(S_{i}^{*}(\mathbf{p})\right)=\mathbf{y}\left(S_{i}^{*}(\mathbf{r})\right)$.

(iii) Let $S_{i}^{*}(\mathbf{p})=\mathbf{p}+\tau_{1} \mathbf{b}$ and $S_{i}^{*}\left(\mathbf{p}+b_{k} e_{k}\right)=\mathbf{p}+b_{k} e_{k}+\tau_{2} \mathbf{b}$ : It is not aggregate-optimal to produce a batch of component $i$ at $\mathbf{x}_{1}=\mathbf{p}+\tau_{1} \mathbf{b}$ and $\mathbf{x}_{2}=\mathbf{p}+b_{k} e_{k}+\tau_{2} \mathbf{b}$. Since $r^{*}$ satisfies Property 2 , it is not aggregate-optimal to produce a batch of component $i$ at $\mathbf{x}_{3}=\mathbf{p}+\tau_{2} \mathbf{b}$, implying $\tau_{2} \geq \tau_{1}$. Hence we must have $S_{i}^{*}\left(\mathbf{p}+b_{k} e_{k}\right) \geq S_{i}^{*}(\mathbf{p})+b_{k} e_{k}$. 
(iv) Let $S_{i}^{*}(\mathbf{p})=\mathbf{p}+\tau_{1} \mathbf{b}$ and $S_{i}^{*}\left(\mathbf{p}+\sum_{k \in I} b_{k} e_{k}\right)=\mathbf{p}+\sum_{k \in I} b_{k} e_{k}+\tau_{2} \mathbf{b}$ : It is not aggregate-optimal to produce a batch of component $i$ at $\mathbf{x}_{1}=\mathbf{p}+\tau_{1} \mathbf{b}$ and $\mathbf{x}_{2}=\mathbf{p}+\sum_{k \in I} b_{k} e_{k}+\tau_{2} \mathbf{b}$. Notice that Properties 1 and 2 imply the following inequalities: $f\left(\mathbf{y}+e_{i}+\sum_{k \in I} e_{k}\right)-f\left(\mathbf{y}+\sum_{k \in I} e_{k}\right) \geq$ $f\left(\mathbf{y}+e_{i}+\mathbf{e}\right)-f(\mathbf{y}+\mathbf{e}) \geq f\left(\mathbf{y}+e_{i}\right)-f(\mathbf{y})$. Thus, since $r^{*}\left(\mathbf{y}+e_{i}+\sum_{k \in I} e_{k}\right)-r^{*}\left(\mathbf{y}+\sum_{k \in I} e_{k}\right) \geq$ $r^{*}\left(\mathbf{y}+e_{i}\right)-r^{*}(\mathbf{y})$, it is not aggregate-optimal to produce a batch of component $i$ at $\mathbf{x}_{3}=\mathbf{p}+$ $\sum_{k \in I} b_{k} e_{k}+\tau_{1} \mathbf{b}$, implying $\tau_{1} \geq \tau_{2}$. Hence we must have $S_{i}^{*}(\mathbf{p})+\sum_{k \in I} b_{k} e_{k} \geq S_{i}^{*}\left(\mathbf{p}+\sum_{k \in I} b_{k} e_{k}\right)$.

(v) Since $\mathbf{p} \geq \mathbf{a}_{j}$ and $\mathbf{y}\left(\mathbf{p}-\mathbf{a}_{j}\right)=\mathbf{y}(\mathbf{p})$, we have $r^{*}(\mathbf{y}(\mathbf{p}+k \mathbf{b}))+c_{j}=r^{*}\left(\mathbf{y}\left(\mathbf{p}+k \mathbf{b}-\mathbf{a}_{j}\right)\right)+c_{j} \geq$ $r^{*}\left(\mathbf{y}\left(\mathbf{p}+k \mathbf{b}-\mathbf{a}_{j}\right)\right), \forall k \in \mathbb{N}_{0}$ : It is always aggregate-optimal to satisfy a demand for product $j$ on each of the lattices $\mathbb{L}(\mathbf{p}, \mathbf{b})$ with $\mathbf{p} \geq \mathbf{a}_{j}$.

(vi) Pick arbitrary $\mathbf{p}$ and $\mathbf{r}$ such that $\mathbf{y}(\mathbf{p})=\mathbf{y}(\mathbf{r})=\mathbf{y}\left(\mathbf{p}+\mathbf{b}-\mathbf{a}_{j}\right)=\mathbf{y}\left(\mathbf{r}+\mathbf{b}-\mathbf{a}_{j}\right)$. Suppose that it is aggregate-optimal to satisfy a demand for product $j$ in original state $\mathbf{x}_{1}=\mathbf{p}+k \mathbf{b}$ : $r^{*}\left(\mathbf{y}\left(\mathbf{p}+k \mathbf{b}-\mathbf{a}_{j}\right)\right)=r^{*}(\mathbf{y}(\mathbf{p})+(k-1) \mathbf{e})<r^{*}(\mathbf{y}(\mathbf{p})+k \mathbf{e})+c_{j}=r^{*}(\mathbf{y}(\mathbf{p}+k \mathbf{b}))+c_{j}$. Notice that it is aggregate-optimal to satisfy a demand for product $j$ in original state $\mathbf{x}_{2}=\mathbf{r}+k \mathbf{b}$ since $r^{*}\left(\mathbf{y}\left(\mathbf{r}+k \mathbf{b}-\mathbf{a}_{j}\right)\right)=r^{*}(\mathbf{y}(\mathbf{r})+(k-1) \mathbf{e})=r^{*}(\mathbf{y}(\mathbf{p})+(k-1) \mathbf{e})<r^{*}(\mathbf{y}(\mathbf{p})+k \mathbf{e})+c_{j}=$ $r^{*}(\mathbf{y}(\mathbf{r})+k \mathbf{e})+c_{j}=r^{*}(\mathbf{y}(\mathbf{r}+k \mathbf{b}))+c_{j}$. Likewise, it can be shown that if it is aggregate-optimal to reject a demand for product $j$ in original state $\mathbf{x}_{1}$, it is aggregate-optimal to reject a demand for product $j$ in original state $\mathbf{x}_{2}$. Hence we must have $\mathbf{y}\left(R_{j}^{*}(\mathbf{p})\right)=\mathbf{y}\left(R_{j}^{*}(\mathbf{r})\right)$.

(vii) Let $R_{j}^{*}(\mathbf{p})=\mathbf{p}+\tau_{1} \mathbf{b}$ and $R_{j}^{*}\left(\mathbf{p}+b_{i} e_{i}\right)=\mathbf{p}+b_{i} e_{i}+\tau_{2} \mathbf{b}$ : It is aggregate-optimal to satisfy a demand for product $j$ at $\mathbf{x}_{1}=\mathbf{p}+\tau_{1} \mathbf{b}$ and $\mathbf{x}_{2}=\mathbf{p}+b_{i} e_{i}+\tau_{2} \mathbf{b}$. Since $r^{*}$ satisfies Property 1 , it is aggregate-optimal to satisfy a demand for product $j$ at $\mathbf{x}_{3}=\mathbf{p}+\tau_{1} \mathbf{b}+b_{i} e_{i}$, implying $\tau_{1} \geq \tau_{2}$. Thus $R_{j}^{*}(\mathbf{p})+b_{i} e_{i} \geq R_{j}^{*}\left(\mathbf{p}+b_{i} e_{i}\right)$.

\section{EC.2. Proofs of the Results in Section 5}

Proposition EC.1. Let $\tau_{j}$ denote an arbitrary positive integer, $\forall j$. Suppose that $a_{i 1}=1, \forall i, a_{i j}=$ $\tau_{1} \times . . \times \tau_{j-1}, \forall i, \forall j>1, c_{j+1} \geq \tau_{j} c_{j}, \forall j<n$, and $\mathbf{q}=\mathbf{a}_{n}$. Also suppose that $X_{i}(0)=0, \forall i$. Then it is always optimal to fulfill a demand for product $n$ if sufficient inventory exists.

Proof of Proposition EC.1. Define the operator $T$ on the set of real-valued functions $v: T v(\mathbf{x})=$ $h(\mathbf{x})+\sum_{i} \mu_{i} T^{(i)} v(\mathbf{x})+\sum_{j} \lambda_{j} T_{j} v(\mathbf{x})$. Assume that the function $v$ satisfies the following properties:

$$
\begin{aligned}
& f(\mathbf{x})-f\left(\mathbf{x}-\mathbf{a}_{j}\right) \geq-c_{j} \text { for } x_{i} \geq a_{i j}+a_{i, j+1}\left\lfloor\frac{x_{i}}{a_{i, j+1}}\right\rfloor, \forall i, \\
& f(\mathbf{x})-f\left(\mathbf{x}-\mathbf{a}_{n}\right) \geq-c_{n} \text { for } \mathbf{x} \geq \mathbf{a}_{n} .
\end{aligned}
$$


We below show that $T v$ satisfies Properties (EC.3) and (EC.4). (If satisfied by the optimal cost function, Property (EC.3) implies that it is optimal to fulfill a demand for any product if the total demand for the next larger product that can be satisfied from on-hand inventory remains the same, and Property (EC.4) implies that it is always optimal to fulfill demands for the largest product if sufficient inventory exists.)

- Assuming $v$ satisfies Properties (EC.3) and (EC.4), first we will show Tv satisfies Property (EC.3). Notice that $T v(\mathbf{x})-T v\left(\mathbf{x}-\mathbf{a}_{j}\right)+c_{j} \geq \sum_{i} \mu_{i}\left(T^{(i)} v(\mathbf{x})-T^{(i)}\left(\mathbf{x}-\mathbf{a}_{j}\right)+c_{j}\right)+$ $\sum_{k} \lambda_{k}\left(T_{k} v(\mathbf{x})-T_{k} v\left(\mathbf{x}-\mathbf{a}_{j}\right)+c_{j}\right)$.

(a) We will show $T^{(i)} v$ satisfies Property (EC.3), i.e., $T^{(i)} v(\mathbf{x})-T^{(i)} v\left(\mathbf{x}-\mathbf{a}_{j}\right)+c_{j} \geq 0$ for $x_{i} \geq a_{i j}+a_{i, j+1}\left\lfloor\frac{x_{i}}{a_{i, j+1}}\right\rfloor, \forall i$.

(1) Suppose that $T^{(i)} v(\mathbf{x})=v(\mathbf{x}) \leq v\left(\mathbf{x}+q_{i} e_{i}\right)$. As we assume $v$ satisfies Property (EC.3), $T^{(i)} v(\mathbf{x})-T^{(i)} v\left(\mathbf{x}-\mathbf{a}_{j}\right)+c_{j} \geq v(\mathbf{x})-v\left(\mathbf{x}-\mathbf{a}_{j}\right)+c_{j} \geq 0$.

(2) Suppose that $T^{(i)} v(\mathbf{x})=v\left(\mathbf{x}+q_{i} e_{i}\right)<v(\mathbf{x})$. Since $q_{i}=a_{i n}$ and $x_{i}+q_{i} \geq a_{i j}+$ $a_{i, j+1}\left\lfloor\frac{x_{i}+q_{i}}{a_{i, j+1}}\right\rfloor$, and as we assume $v$ satisfies Property (EC.3), $T^{(i)} v(\mathbf{x})-T^{(i)} v(\mathbf{x}-$ $\left.\mathbf{a}_{j}\right)+c_{j} \geq v\left(\mathbf{x}+q_{i} e_{i}\right)-v\left(\mathbf{x}+q_{i} e_{i}-\mathbf{a}_{j}\right)+c_{j} \geq 0$.

(b) We will show $T_{k} v$ satisfies Property (EC.3) for $k<j$, i.e., $T_{k} v(\mathbf{x})-T_{k} v\left(\mathbf{x}-\mathbf{a}_{j}\right)+c_{j} \geq 0$ for $k<j$ and $x_{i} \geq a_{i j}+a_{i, j+1}\left\lfloor\frac{x_{i}}{a_{i, j+1}}\right\rfloor, \forall i$. (Recall that $\mathbf{a}_{k}<\mathbf{a}_{j}$ if $k<j$.)

(1) Suppose that $T_{k} v(\mathbf{x})=v(\mathbf{x})+c_{k}<v\left(\mathbf{x}-\mathbf{a}_{k}\right)$. As we assume $v$ satisfies Property (EC.3), $T_{k} v(\mathbf{x})-T_{k} v\left(\mathbf{x}-\mathbf{a}_{j}\right)+c_{j} \geq v(\mathbf{x})+c_{k}-v\left(\mathbf{x}-\mathbf{a}_{j}\right)-c_{k}+c_{j} \geq 0$.

(2) Suppose that $T_{k} v(\mathbf{x})=v\left(\mathbf{x}-\mathbf{a}_{k}\right) \leq v(\mathbf{x})+c_{k}$ and $x_{i} \geq a_{i k}+a_{i j}\left\lfloor\frac{x_{i}}{a_{i j}}\right\rfloor, \forall i$. As we consider states obeying $x_{i} \geq a_{i j}+a_{i, j+1}\left\lfloor\frac{x_{i}}{a_{i, j+1}}\right\rfloor, \forall i$, we must have $x_{i} \geq a_{i k}+a_{i j}$ and $x_{i}-a_{i k} \geq a_{i j}+a_{i, j+1}\left\lfloor\frac{x_{i}-a_{i k}}{a_{i, j+1}}\right\rfloor, \forall i$. As we assume $v$ satisfies Property (EC.3), $T_{k} v(\mathbf{x})-T_{k} v\left(\mathbf{x}-\mathbf{a}_{j}\right)+c_{j} \geq v\left(\mathbf{x}-\mathbf{a}_{k}\right)-v\left(\mathbf{x}-\mathbf{a}_{j}-\mathbf{a}_{k}\right)+c_{j} \geq 0$.

(3) Suppose that $T_{k} v(\mathbf{x})=v\left(\mathbf{x}-\mathbf{a}_{k}\right) \leq v(\mathbf{x})+c_{k}$ and $\exists i$ such that $x_{i}<a_{i k}+a_{i j}\left\lfloor\frac{x_{i}}{a_{i j}}\right\rfloor$. Note that we should have $\mathbf{x} \geq \mathbf{a}_{k}$ for this case to exist. If $x_{i}<a_{i k}+a_{i j}\left\lfloor\frac{x_{i}}{a_{i j}}\right\rfloor$, notice that $x_{i}=y_{i} a_{i j}+z_{1}$ where $y_{i} \geq 1$ and $0 \leq z_{1}<a_{i k}$. Our assumptions of $a_{i j}=a_{k j}, \forall i, k, j$, $q_{i}=a_{i n}, \forall i$, and $X_{i}(0)=0, \forall i$, imply that $\left|x_{i}-x_{k}\right|$ is zero or an integer multiple of $a_{i n}$ at any time, $\forall i, k$. Thus, if $\exists i$ such that $x_{i}<a_{i k}+a_{i j}\left\lfloor\frac{x_{i}}{a_{i j}}\right\rfloor$, we must have $x_{i}=y_{i} a_{i j}+z_{1}$ for all $i$ where $y_{i} \geq 1$ and $0 \leq z_{1}<a_{i k}$. Notice that $x_{i}-a_{i k}=\left(y_{i}-1\right) a_{i j}+z_{2}$ where $z_{2}=z_{1}+a_{i j}-a_{i k}<a_{i j}, \forall i$ : Because we have $z_{2} \geq a_{i j}-a_{i k}=a_{i j}-a_{i, k+1}+\left(\tau_{k}-1\right) a_{i k}$, the system at state $\mathbf{x}-\mathbf{a}_{k}$ can fulfill $\tau_{k}-1$ demands for product $k$ (and move to 
state $\left.\mathbf{x}-\mathbf{a}_{k}-\left(\tau_{k}-1\right) \mathbf{a}_{k}=\mathbf{x}-\mathbf{a}_{k+1}\right)$ without reducing the total demand for product $k+1$ that can be satisfied from on-hand inventory. Now notice that $x_{i}-a_{i, k+1}=$ $\left(y_{i}-1\right) a_{i j}+z_{3}$ where $z_{3}=z_{1}+a_{i j}-a_{i, k+1}<a_{i j}, \forall i$ : Because we have $z_{3} \geq a_{i j}-a_{i, k+1}=$ $a_{i j}-a_{i, k+2}+\left(\tau_{k+1}-1\right) a_{i, k+1}$, the system at state $\mathbf{x}-\mathbf{a}_{k+1}$ can fulfill $\tau_{k+1}-1$ demands for product $k+1$ (and move to state $\mathbf{x}-\mathbf{a}_{k+1}-\left(\tau_{k+1}-1\right) \mathbf{a}_{k+1}=\mathbf{x}-\mathbf{a}_{k+2}$ ) without reducing the total demand for product $k+2$ that can be satisfied from on-hand inventory. This procedure can be repeated until the system reaches state $\mathbf{x}-\mathbf{a}_{j}$. As we assume $v$ satisfies Property (EC.3), the following inequalities hold:

$$
\begin{gathered}
\left(\tau_{k}-1\right) \text { inequalities }\left\{\begin{array}{c}
v\left(\mathbf{x}-\mathbf{a}_{k}\right)-v\left(\mathbf{x}-2 \mathbf{a}_{k}\right) \geq-c_{k} \\
v\left(\mathbf{x}-2 \mathbf{a}_{k}\right)-v\left(\mathbf{x}-3 \mathbf{a}_{k}\right) \geq-c_{k} \\
\vdots \\
v\left(\mathbf{x}-\left(\tau_{k}-1\right) \mathbf{a}_{k}\right)-v\left(\mathbf{x}-\mathbf{a}_{k+1}\right) \geq-c_{k}
\end{array}\right. \\
\left(\tau_{k+1}-1\right) \text { inequalities }\left\{\begin{array}{c}
v\left(\mathbf{x}-\mathbf{a}_{k+1}\right)-v\left(\mathbf{x}-2 \mathbf{a}_{k+1}\right) \geq-c_{k+1} \\
v\left(\mathbf{x}-2 \mathbf{a}_{k+1}\right)-v\left(\mathbf{x}-3 \mathbf{a}_{k+1}\right) \geq-c_{k+1} \\
\vdots \\
v\left(\mathbf{x}-\left(\tau_{k+1}-1\right) \mathbf{a}_{k+1}\right)-v\left(\mathbf{x}-\mathbf{a}_{k+2}\right) \geq-c_{k+1} \\
\vdots \\
\left(\tau_{j-1}-1\right) \text { inequalities }\left\{\begin{array}{c}
v\left(\mathbf{x}-\mathbf{a}_{j-1}\right)-v\left(\mathbf{x}-2 \mathbf{a}_{j-1}\right) \geq-c_{j-1} \\
v\left(\mathbf{x}-2 \mathbf{a}_{j-1}\right)-v\left(\mathbf{x}-3 \mathbf{a}_{j-1}\right) \geq-c_{j-1} \\
\vdots \\
v\left(\mathbf{x}-\left(\tau_{j-1}-1\right) \mathbf{a}_{j-1}\right)-v\left(\mathbf{x}-\mathbf{a}_{j}\right) \geq-c_{j-1}
\end{array}\right.
\end{array}\right.
\end{gathered}
$$

Summation of the above inequalities implies that $v\left(\mathbf{x}-\mathbf{a}_{k}\right)-v\left(\mathbf{x}-\mathbf{a}_{j}\right)-c_{k}+c_{j} \geq$ $-c_{k}\left(\tau_{k}-1\right)-c_{k+1}\left(\tau_{k+1}-1\right)-. .-c_{j-2}\left(\tau_{j-2}-1\right)-c_{j-1}\left(\tau_{j-1}-1\right)-c_{k}+c_{j}=-c_{j-1} \tau_{j-1}+$ $\left(c_{j-1}-c_{j-2} \tau_{j-2}\right)+\left(c_{j-2}-c_{j-3} \tau_{j-3}\right)+. .+\left(c_{k+1}-c_{k} \tau_{k}\right)+c_{k}-c_{k}+c_{j} \geq 0$. The last inequality follows from our assumption of $c_{j+1} \geq \tau_{j} c_{j}, \forall j<n$. As we assume $T_{k} v(\mathbf{x})=$ $v\left(\mathbf{x}-\mathbf{a}_{k}\right), T_{k} v(\mathbf{x})-T_{k} v\left(\mathbf{x}-\mathbf{a}_{j}\right)+c_{j} \geq v\left(\mathbf{x}-\mathbf{a}_{k}\right)-v\left(\mathbf{x}-\mathbf{a}_{j}\right)-c_{k}+c_{j} \geq 0$.

(c) We will show $T_{j} v$ satisfies Property (EC.3), i.e., $T_{j} v(\mathbf{x})-T_{j} v\left(\mathbf{x}-\mathbf{a}_{j}\right)+c_{j} \geq 0$ for $x_{i} \geq$ $a_{i j}+a_{i, j+1}\left\lfloor\frac{x_{i}}{a_{i, j+1}}\right\rfloor, \forall i$. As we assume $v$ satisfies Property (EC.3), $T_{j} v(\mathbf{x})=v\left(\mathbf{x}-\mathbf{a}_{j}\right) \leq$ $v(\mathbf{x})+c_{j}$. Thus $T_{j} v(\mathbf{x})-T_{j} v\left(\mathbf{x}-\mathbf{a}_{j}\right)+c_{j} \geq v\left(\mathbf{x}-\mathbf{a}_{j}\right)-v\left(\mathbf{x}-\mathbf{a}_{j}\right)-c_{j}+c_{j}=0$.

(d) We will show $T_{k} v$ satisfies Property (EC.3) for $k>j$, i.e., $T_{k} v(\mathbf{x})-T_{k} v\left(\mathbf{x}-\mathbf{a}_{j}\right)+c_{j} \geq 0$ for $k>j$ and $x_{i} \geq a_{i j}+a_{i, j+1}\left\lfloor\frac{x_{i}}{a_{i, j+1}}\right\rfloor, \forall i$.

(1) Suppose that $T_{k} v(\mathbf{x})=v(\mathbf{x})+c_{k}$. As we assume $v$ satisfies Property (EC.3), $T_{k} v(\mathbf{x})-$ $T_{k} v\left(\mathbf{x}-\mathbf{a}_{j}\right)+c_{j} \geq v(\mathbf{x})+c_{k}-v\left(\mathbf{x}-\mathbf{a}_{j}\right)-c_{k}+c_{j} \geq 0$. 
(2) Suppose that $\mathbf{x} \geq \mathbf{a}_{k}$ and $T_{k} v(\mathbf{x})=v\left(\mathbf{x}-\mathbf{a}_{k}\right) \leq v(\mathbf{x})+c_{k}$. Since $x_{i}-a_{i k} \geq a_{i j}+$ $a_{i, j+1}\left\lfloor\frac{x_{i}-a_{i k}}{a_{i, j+1}}\right\rfloor, \forall i$, and as we assume $v$ satisfies Property (EC.3), $T_{k} v(\mathbf{x})-T_{k} v(\mathbf{x}-$ $\left.\mathbf{a}_{j}\right)+c_{j} \geq v\left(\mathbf{x}-\mathbf{a}_{k}\right)-v\left(\mathbf{x}-\mathbf{a}_{j}-\mathbf{a}_{k}\right)+c_{j} \geq 0$.

Because $\sum_{i} \mu_{i}\left(T^{(i)} v(\mathbf{x})-T^{(i)}\left(\mathbf{x}-\mathbf{a}_{j}\right)+c_{j}\right)+\sum_{k} \lambda_{k}\left(T_{k} v(\mathbf{x})-T_{k} v\left(\mathbf{x}-\mathbf{a}_{j}\right)+c_{j}\right) \geq 0$ for $x_{i} \geq$ $a_{i j}+a_{i, j+1}\left\lfloor\frac{x_{i}}{a_{i, j+1}}\right\rfloor, \forall i, T v$ satisfies Property (EC.3).

- Assuming $v$ satisfies Properties (EC.3) and (EC.4), next we will show Tv satisfies Property (EC.4). Notice that $T v(\mathbf{x})-T v\left(\mathbf{x}-\mathbf{a}_{n}\right)+c_{n} \geq \sum_{i} \mu_{i}\left(T^{(i)} v(\mathbf{x})-T^{(i)}\left(\mathbf{x}-\mathbf{a}_{n}\right)+c_{n}\right)+$ $\sum_{k} \lambda_{k}\left(T_{k} v(\mathbf{x})-T_{k} v\left(\mathbf{x}-\mathbf{a}_{n}\right)+c_{n}\right)$.

(a) We will show $T^{(i)} v$ satisfies Property (EC.4), i.e., $T^{(i)} v(\mathbf{x})-T^{(i)} v\left(\mathbf{x}-\mathbf{a}_{n}\right)+c_{n} \geq 0$.

(1) Suppose that $T^{(i)} v(\mathbf{x})=v\left(\mathbf{x}+q_{i} e_{i}\right)<v(\mathbf{x})$. As we assume $v$ satisfies Property (EC.4), $T^{(i)} v(\mathbf{x})-T^{(i)} v\left(\mathbf{x}-\mathbf{a}_{n}\right)+c_{n} \geq v\left(\mathbf{x}+q_{i} e_{i}\right)-v\left(\mathbf{x}+q_{i} e_{i}-\mathbf{a}_{n}\right)+c_{n} \geq 0$.

(2) Suppose that $T^{(i)} v(\mathbf{x})=v(\mathbf{x}) \leq v\left(\mathbf{x}+q_{i} e_{i}\right)$. As we assume $v$ satisfies Property (EC.4), $T^{(i)} v(\mathbf{x})-T^{(i)} v\left(\mathbf{x}-\mathbf{a}_{n}\right)+c_{n} \geq v(\mathbf{x})-v\left(\mathbf{x}-\mathbf{a}_{n}\right)+c_{n} \geq 0$.

(b) We will show $T_{k} v$ satisfies Property (EC.4) for $k<n$, i.e., $T_{k} v(\mathbf{x})-T_{k} v\left(\mathbf{x}-\mathbf{a}_{n}\right)+c_{n} \geq 0$ for $k<n$.

(1) Suppose that $T_{k} v(\mathbf{x})=v(\mathbf{x})+c_{k}<v\left(\mathbf{x}-\mathbf{a}_{k}\right)$. As we assume $v$ satisfies Property (EC.4), $T_{k} v(\mathbf{x})-T_{k} v\left(\mathbf{x}-\mathbf{a}_{n}\right)+c_{n} \geq v(\mathbf{x})+c_{k}-v\left(\mathbf{x}-\mathbf{a}_{n}\right)-c_{k}+c_{n} \geq 0$.

(2) Suppose that $T_{k} v(\mathbf{x})=v\left(\mathbf{x}-\mathbf{a}_{k}\right) \leq v(\mathbf{x})+c_{k}$ and $x_{i} \geq a_{i k}+a_{i n}\left\lfloor\frac{x_{i}}{a_{i n}}\right\rfloor, \forall i$. Since $x_{i} \geq$ $a_{i n}$, we have $x_{i} \geq a_{i k}+a_{i n}$ in this case. As we assume $v$ satisfies Property (EC.4), $T_{k} v(\mathbf{x})-T_{k} v\left(\mathbf{x}-\mathbf{a}_{n}\right)+c_{n} \geq v\left(\mathbf{x}-\mathbf{a}_{k}\right)-v\left(\mathbf{x}-\mathbf{a}_{n}-\mathbf{a}_{k}\right)+c_{n} \geq 0$.

(3) Suppose that $T_{k} v(\mathbf{x})=v\left(\mathbf{x}-\mathbf{a}_{k}\right) \leq v(\mathbf{x})+c_{k}$ and $\exists i$ such that $x_{i}<a_{i k}+a_{i n}\left\lfloor\frac{x_{i}}{a_{i n}}\right\rfloor$. Taking the same steps in case (3) of part (b) that we considered to prove Property (EC.3), but replacing $j$ with $n$, it is easy to verify that $T_{k} v(\mathbf{x})-T_{k} v\left(\mathbf{x}-\mathbf{a}_{n}\right)+c_{n} \geq 0$. (c) We will show $T_{n} v$ satisfies Property (EC.4), i.e., $T_{n} v(\mathbf{x})-T_{n} v\left(\mathbf{x}-\mathbf{a}_{n}\right)+c_{n} \geq 0$. As we assume $v$ satisfies Property (EC.4), $T_{n} v(\mathbf{x})=v\left(\mathbf{x}-\mathbf{a}_{n}\right) \leq v(\mathbf{x})+c_{n}$. Thus $T_{n} v(\mathbf{x})-T_{n} v(\mathbf{x}-$ $\left.\mathbf{a}_{n}\right)+c_{n} \geq v\left(\mathbf{x}-\mathbf{a}_{n}\right)-v\left(\mathbf{x}-\mathbf{a}_{n}\right)-c_{n}+c_{n}=0$.

Because $\sum_{i} \mu_{i}\left(T^{(i)} v(\mathbf{x})-T^{(i)}\left(\mathbf{x}-\mathbf{a}_{n}\right)+c_{n}\right)+\sum_{k} \lambda_{k}\left(T_{k} v(\mathbf{x})-T_{k} v\left(\mathbf{x}-\mathbf{a}_{n}\right)+c_{n}\right) \geq 0, T v$ satisfies Property (EC.4).

Following Propositions 3.1.5 and 3.1.6 in Bertsekas (2007), we verify that $\lim _{k \rightarrow \infty}\left(T^{k} v_{0}\right)(\mathbf{x})=$ $v^{*}(\mathbf{x})$ where $v_{0}$ is the zero function, $v^{*}$ is the optimal cost function, and $T^{k}$ refers to $k$ compositions of operator T. Because $v_{0}$ satisfies Properties (EC.3) and (EC.4), and if $v$ satisfies Properties (EC.3) 
and (EC.4), then Tv satisfies Properties (EC.3) and (EC.4), $v^{*}$ also satisfies Properties (EC.3) and (EC.4). Hence, by Property (EC.4), it is always optimal to fulfill a demand for product $n$ if sufficient inventory is available.

Proof of Lemma 2. First we will prove $v^{*}\left(\mathbf{x}_{1}\right)-v^{*}\left(\mathbf{x}_{2}\right) \leq \frac{h\left(\mathbf{x}_{1}\right)-h\left(\mathbf{x}_{2}\right)}{\alpha}, \forall \mathbf{x}_{1} \geq \mathbf{x}_{2}$. Assuming that $v\left(\mathbf{x}_{2}\right)+\frac{h\left(\mathbf{x}_{1}\right)-h\left(\mathbf{x}_{2}\right)}{\alpha} \geq v\left(\mathbf{x}_{1}\right)$, we want to show $T v\left(\mathbf{x}_{2}\right)+\frac{h\left(\mathbf{x}_{1}\right)-h\left(\mathbf{x}_{2}\right)}{\alpha} \geq T v\left(\mathbf{x}_{1}\right)$.

- Assuming that $v\left(\mathbf{x}_{2}\right)+\frac{h\left(\mathbf{x}_{1}\right)-h\left(\mathbf{x}_{2}\right)}{\alpha} \geq v\left(\mathbf{x}_{1}\right)$, below we show $T^{(i)} v\left(\mathbf{x}_{2}\right)+\frac{h\left(\mathbf{x}_{1}\right)-h\left(\mathbf{x}_{2}\right)}{\alpha} \geq T^{(i)} v\left(\mathbf{x}_{1}\right)$.

(1) Suppose that $T^{(i)} v\left(\mathbf{x}_{2}\right)=v\left(\mathbf{x}_{2}+q_{i} e_{i}\right)<v\left(\mathbf{x}_{2}\right)$. Then $T^{(i)} v\left(\mathbf{x}_{2}\right)+\frac{h\left(\mathbf{x}_{1}\right)-h\left(\mathbf{x}_{2}\right)}{\alpha}=v\left(\mathbf{x}_{2}+q_{i} e_{i}\right)+$ $\frac{h\left(\mathbf{x}_{1}\right)-h\left(\mathbf{x}_{2}\right)}{\alpha}=v\left(\mathbf{x}_{2}+q_{i} e_{i}\right)+\frac{h\left(\mathbf{x}_{1}+q_{i} e_{i}\right)-h\left(\mathbf{x}_{2}+q_{i} e_{i}\right)}{\alpha} \geq v\left(\mathbf{x}_{1}+q_{i} e_{i}\right) \geq T^{(i)} v\left(\mathbf{x}_{1}\right)$.

(2) Suppose that $T^{(i)} v\left(\mathbf{x}_{2}\right)=v\left(\mathbf{x}_{2}\right)<v\left(\mathbf{x}_{2}+q_{i} e_{i}\right)$. Then $T^{(i)} v\left(\mathbf{x}_{2}\right)+\frac{h\left(\mathbf{x}_{1}\right)-h\left(\mathbf{x}_{2}\right)}{\alpha}=v\left(\mathbf{x}_{2}\right)+$ $\frac{h\left(\mathbf{x}_{1}\right)-h\left(\mathbf{x}_{2}\right)}{\alpha} \geq v\left(\mathbf{x}_{1}\right) \geq T^{(i)} v\left(\mathbf{x}_{1}\right)$.

- Assuming that $v\left(\mathbf{x}_{2}\right)+\frac{h\left(\mathbf{x}_{1}\right)-h\left(\mathbf{x}_{2}\right)}{\alpha} \geq v\left(\mathbf{x}_{1}\right)$, below we show $T_{j} v\left(\mathbf{x}_{2}\right)+\frac{h\left(\mathbf{x}_{1}\right)-h\left(\mathbf{x}_{2}\right)}{\alpha} \geq T_{j} v\left(\mathbf{x}_{1}\right)$.

(1) Suppose that $T_{j} v\left(\mathbf{x}_{2}\right)=v\left(\mathbf{x}_{2}-\mathbf{a}_{j}\right)<v\left(\mathbf{x}_{2}\right)+c_{j}$. Then $T_{j} v\left(\mathbf{x}_{2}\right)+\frac{h\left(\mathbf{x}_{1}\right)-h\left(\mathbf{x}_{2}\right)}{\alpha}=v\left(\mathbf{x}_{2}-\mathbf{a}_{j}\right)+$ $\frac{h\left(\mathbf{x}_{1}\right)-h\left(\mathbf{x}_{2}\right)}{\alpha}=v\left(\mathbf{x}_{2}-\mathbf{a}_{j}\right)+\frac{h\left(\mathbf{x}_{1}-\mathbf{a}_{j}\right)-h\left(\mathbf{x}_{2}-\mathbf{a}_{j}\right)}{\alpha} \geq v\left(\mathbf{x}_{1}-\mathbf{a}_{j}\right) \geq T_{j} v\left(\mathbf{x}_{1}\right)$.

(2) Suppose that $T_{j} v\left(\mathbf{x}_{2}\right)=v\left(\mathbf{x}_{2}\right)+c_{j}<v\left(\mathbf{x}_{2}-\mathbf{a}_{j}\right)$. Then $T_{j} v\left(\mathbf{x}_{2}\right)+\frac{h\left(\mathbf{x}_{1}\right)-h\left(\mathbf{x}_{2}\right)}{\alpha}=v\left(\mathbf{x}_{2}\right)+c_{j}+$ $\frac{h\left(\mathbf{x}_{1}\right)-h\left(\mathbf{x}_{2}\right)}{\alpha} \geq v\left(\mathbf{x}_{1}\right)+c_{j} \geq T_{j} v\left(\mathbf{x}_{1}\right)$.

Notice that $T v\left(\mathbf{x}_{1}\right)-T v\left(\mathbf{x}_{2}\right)=h\left(\mathbf{x}_{1}\right)-h\left(\mathbf{x}_{2}\right)+\sum_{i} \mu_{i}\left(T^{(i)} v\left(\mathbf{x}_{1}\right)-T^{(i)} v\left(\mathbf{x}_{2}\right)\right)+\sum_{j} \lambda_{j}\left(T_{j} v\left(\mathbf{x}_{1}\right)-\right.$ $\left.T_{j} v\left(\mathbf{x}_{2}\right)\right) \leq h\left(\mathbf{x}_{1}\right)-h\left(\mathbf{x}_{2}\right)+\left(\sum_{i} \mu_{i}+\sum_{j} \lambda_{j}\right)\left(\frac{h\left(\mathbf{x}_{1}\right)-h\left(\mathbf{x}_{2}\right)}{\alpha}\right)=h\left(\mathbf{x}_{1}\right)-h\left(\mathbf{x}_{2}\right)+(1-\alpha)\left(\frac{h\left(\mathbf{x}_{1}\right)-h\left(\mathbf{x}_{2}\right)}{\alpha}\right)=$ $\frac{h\left(\mathbf{x}_{1}\right)-h\left(\mathbf{x}_{2}\right)}{\alpha}$. Following Propositions 3.1.5 and 3.1.6 in Bertsekas (2007), we verify that $\lim _{k \rightarrow \infty}\left(T^{k} v_{0}\right)(\mathbf{x})=v^{*}(\mathbf{x})$ where $v_{0}$ is the zero function, $v^{*}$ is the optimal cost function, and $T^{k}$ refers to $k$ compositions of operator $T$. Since $v_{0}\left(\mathbf{x}_{1}\right)-v_{0}\left(\mathbf{x}_{2}\right) \leq \frac{h\left(\mathbf{x}_{1}\right)-h\left(\mathbf{x}_{2}\right)}{\alpha}$, we have $\left(T^{k} v_{0}\right)\left(\mathbf{x}_{1}\right)-$ $\left(T^{k} v_{0}\right)\left(\mathbf{x}_{2}\right) \leq \frac{h\left(\mathbf{x}_{1}\right)-h\left(\mathbf{x}_{2}\right)}{\alpha}$, and therefore $v^{*}\left(\mathbf{x}_{1}\right)-v^{*}\left(\mathbf{x}_{2}\right) \leq \frac{h\left(\mathbf{x}_{1}\right)-h\left(\mathbf{x}_{2}\right)}{\alpha}, \forall \mathbf{x}_{1} \geq \mathbf{x}_{2}$.

By definition of $\tau, \mathbf{x}_{1}+\tau \mathbf{a}_{j^{*}} \geq \mathbf{x}_{2}$. Thus:

$$
v^{*}\left(\mathbf{x}_{1}+\tau \mathbf{a}_{j^{*}}\right)-v^{*}\left(\mathbf{x}_{2}\right) \leq \frac{h\left(\mathbf{x}_{1}+\tau \mathbf{a}_{j^{*}}\right)-h\left(\mathbf{x}_{2}\right)}{\alpha} .
$$

When $\tau=0$, i.e. $\mathbf{x}_{1} \geq \mathbf{x}_{2}$, the above inequality implies Lemma 2 . As we assume that it is always optimal to satisfy a demand for product $j^{*}$, when $\tau>0$ it is easy to verify that

$$
\begin{gathered}
v^{*}\left(\mathbf{x}_{1}\right)-v^{*}\left(\mathbf{x}_{1}+\mathbf{a}_{j^{*}}\right) \leq c_{j^{*}} \\
v^{*}\left(\mathbf{x}_{1}+\mathbf{a}_{j^{*}}\right)-v^{*}\left(\mathbf{x}_{1}+2 \mathbf{a}_{j^{*}}\right) \leq c_{j^{*}} \\
\vdots \\
v^{*}\left(\mathbf{x}_{1}+(\tau-1) \mathbf{a}_{j^{*}}\right)-v^{*}\left(\mathbf{x}_{1}+\tau \mathbf{a}_{j^{*}}\right) \leq c_{j^{*}}
\end{gathered}
$$


Summation of these inequalities implies that

$$
v^{*}\left(\mathbf{x}_{1}\right)-v^{*}\left(\mathbf{x}_{1}+\tau \mathbf{a}_{j^{*}}\right) \leq \tau c_{j^{*}}
$$

Summation of inequalities (EC.5) and (EC.6) yields $v^{*}\left(\mathbf{x}_{1}\right)-v^{*}\left(\mathbf{x}_{2}\right) \leq \frac{h\left(\mathbf{x}_{1}+\tau \mathbf{a}_{j^{*}}\right)-h\left(\mathbf{x}_{2}\right)}{\alpha}+\tau c_{j^{*}}$.

Proof of Theorem 2. By Lemma 2, it is easy to verify that

$$
\left|v^{*}\left(\mathbf{x}_{1}\right)-v^{*}\left(\mathbf{x}_{2}\right)\right| \leq \max \left\{\frac{h\left(\mathbf{x}_{1}+\tau_{1} \mathbf{a}_{j^{*}}-\mathbf{x}_{2}\right)}{\alpha}+\tau_{1} c_{j^{*}}, \frac{h\left(\mathbf{x}_{2}+\tau_{2} \mathbf{a}_{j^{*}}-\mathbf{x}_{1}\right)}{\alpha}+\tau_{2} c_{j^{*}}\right\}
$$

where $\tau_{i}=\min \left\{k \in \mathbb{N}_{0}: \mathbf{x}_{i}+k \mathbf{a}_{j^{*}} \geq \mathbf{x}_{3-i}\right\}, \forall i=1,2$. Tsitsiklis and Van Roy (1996) derived bounds on the error between the optimal cost function $v^{*}$ and its approximation $r^{*}$ under hard aggregation. Specifically, they showed that

$$
r^{*}(\mathbf{y})-\epsilon \leq v^{*}(\mathbf{x}) \leq r^{*}(\mathbf{y})+\epsilon, \quad \forall \mathbf{y} \in \mathcal{Y}, \quad \mathbf{x} \in \mathbf{y}
$$

where

$$
\epsilon=\frac{\max _{\mathbf{y} \in \mathcal{Y}} \max _{\mathbf{x}_{1}, \mathbf{x}_{2} \in \mathbf{y}}\left|v^{*}\left(\mathbf{x}_{1}\right)-v^{*}\left(\mathbf{x}_{2}\right)\right|}{\alpha} .
$$

Thus, following Tsitsiklis and Van Roy (1996), it can be shown that

$$
r^{*}(\mathbf{y})-\epsilon \leq v^{*}(\mathbf{x}) \leq r^{*}(\mathbf{y})+\epsilon, \quad \forall \mathbf{y} \in \mathcal{Y}, \quad \mathbf{x} \in \mathbf{y}
$$

where

$$
\epsilon=\max _{\mathbf{y} \in \mathcal{Y}} \max _{\mathbf{x}_{1}, \mathbf{x}_{2} \in \mathbf{y}}\left\{\max \left\{\frac{h\left(\mathbf{x}_{1}+\tau_{1} \mathbf{a}_{j^{*}}-\mathbf{x}_{2}\right)+\alpha \tau_{1} c_{j^{*}}}{\alpha^{2}}, \frac{h\left(\mathbf{x}_{2}+\tau_{2} \mathbf{a}_{j^{*}}-\mathbf{x}_{1}\right)+\alpha \tau_{2} c_{j^{*}}}{\alpha^{2}}\right\}\right\} .
$$

Under our aggregation scheme, notice that the maximum of the bounds in (EC.7) across all pairs of original states $\mathbf{x}_{1}$ and $\mathbf{x}_{2}$ in a particular aggregate state is also the maximum in every other aggregate state. This is because the bound in (EC.7) is based on the difference between states $\mathbf{x}_{1}$ and $\mathbf{x}_{2}$, and the set of all possible values for this difference is the same across different aggregate states. Thus, taking the aggregate state $\mathbf{y}=(0, . ., 0)$, our error bound $\epsilon$ can be written as

$$
\epsilon=\max _{\substack{\mathbf{x}_{1}, \mathbf{x}_{2} \\ \text { s.t. } 0 \leq x_{1, i}, x_{2, i}<b_{i}, \forall i}} \frac{h\left(\mathbf{x}_{1}+\tau \mathbf{a}_{j^{*}}-\mathbf{x}_{2}\right)+\alpha \tau c_{j^{*}}}{\alpha^{2}}
$$

where

$$
\tau=\min \left\{k \in \mathbb{N}_{0}: \mathbf{x}_{1}+k \mathbf{a}_{j^{*}} \geq \mathbf{x}_{2}\right\} .
$$

Proof of Theorem 3. Theorem 2 implies that $v^{*}(\mathbf{x})-\epsilon \leq r^{*}(\mathbf{y}(\mathbf{x})) \leq v^{*}(\mathbf{x})+\epsilon, \forall \mathbf{x}$. Following Lemmas 1.1.1 and 1.1.2 in Bertsekas (2007), we verify that

$$
\left(T^{k} v^{*}\right)(\mathbf{x})-(1-\alpha)^{k} \epsilon \leq\left(T^{k} v\right)(\mathbf{x}) \leq\left(T^{k} v^{*}\right)(\mathbf{x})+(1-\alpha)^{k} \epsilon, \quad \forall k>0,
$$

where $1-\alpha$ is the discount factor, and $v(\mathbf{x})=r^{*}(\mathbf{y}(\mathbf{x})), \forall \mathbf{x}$. Note that $T^{k} v^{*}=v^{*}, \forall k>0$. Taking the limit as $k \rightarrow \infty$ in the preceding relation, we obtain $\lim _{k \rightarrow \infty}\left(T^{k} v\right)(\mathbf{x})=v^{*}(\mathbf{x}), \forall \mathbf{x}$. 


\section{References}

Bertsekas, D. P. 2007. Dynamic Programming and Optimal Control, Vol. 2. Athena Scientific, Nashua, NH.

Tsitsiklis, J. N., B. Van Roy. 1996. Feature-based methods for large scale dynamic programming. Mach. Learn. 22 59-94. 